Florida International University FIU Digital Commons

7-13-2010

\title{
Modeling of Loose Contamination Scenarios to Predict the Amount of Contamination Removed
}

Duriem Calderin Morales

Florida International University, dcald005@fiu.edu

DOI: $10.25148 /$ etd.FI10080409

Follow this and additional works at: https://digitalcommons.fiu.edu/etd

Part of the Other Biomedical Engineering and Bioengineering Commons

\section{Recommended Citation}

Calderin Morales, Duriem, "Modeling of Loose Contamination Scenarios to Predict the Amount of Contamination Removed" (2010). FIU Electronic Theses and Dissertations. 228.

https://digitalcommons.fiu.edu/etd/228 


\section{FLORIDA INTERNATIONAL UNIVERSITY}

Miami, Florida

MODELING OF LOOSE CONTAMINATION SCENARIOS TO PREDICT THE AMOUNT OF CONTAMINATION REMOVED

A thesis submitted in partial fulfillment of the

requirements for the degree of

MASTER OF SCIENCE

in

BIOMEDICAL ENGINEERING

by

Duriem Calderin Morales

2010 
To: Dean Amir Mirmiran

College of Engineering and Computing

This thesis, written by Duriem Calderin Morales, and entitled Modeling of Loose Contamination Scenarios to Predict the Amount of Contamination Removed, having been approved in respect to style and intellectual content, is referred to you for judgment.

We have read this thesis and recommend that it be approved.

Leonel E. Lagos

Chenzhong Li

Anthony McGoron, Major Professor

Date of Defense: July 13, 2010

The thesis of Duriem Calderin Morales is approved.

Dean Amir Mirmiran
College of Engineering and Computing

Interim Dean Kevin O'Shea

University Graduate School

Florida International University, 2010 


\section{DEDICATION}

This thesis is dedicated to those who have supported me through my personal and professional life. Those are my friends, family and especially my parents. 


\section{ACKNOWLEDGMENTS}

The completion of this work would not have been possible without the support and help of my professors and friends. My appreciation goes to my advisor at the Applied Research Center of Florida International University, Dr. Leonel E. Lagos, for presenting me with the opportunity to work on this research project, for granting me the opportunity to be a DOE fellow and for his sponsorship through my Master's study. I also wish to thank my committee members: Dr. Anthony Mc.Goron for his guidance and insightful comments and Dr. Chenzhong Li.

A special thanks to Alejandro Amor for his original ideas, constant encouragement, support, and lab assistance. My appreciation is also extended to Dr. Surendra Dua for his expert advice and valuable insights on this project. Finally, I would like to thank the DOE-FIU Science and Technology Workforce Development Initiative program and the Department of Biomedical Engineering of Florida International University for providing a friendly work environment in which I could learn from others. 
ABSTRACT OF THE THESIS

MODELING OF LOOSE CONTAMINATION SCENARIOS TO PREDICT THE

AMOUNT OF CONTAMINATION REMOVED

\author{
by \\ Duriem Calderin Morales \\ Florida International University, 2010 \\ Miami, Florida \\ Professor Anthony McGoron, Major Professor
}

The objective of this research is to evaluate the influence of the factors identified by the Johnson, Kendall and Robert's theory that affect the strength of the detachment force necessary to remove a particle of contaminant from a surface, and the roughness of the surface in which the contaminant is present, on predicting the efficiency of removal of loose contamination. Two methods were used to reach this objective: the first method consisted of quantifying the contamination by weight and the second method of quantifying the contamination by counting alpha and gamma particles. As a result, it was determined that for particles of $5 \mu \mathrm{m}$, the interaction between contaminant-wipe and contaminant-surface were significant. However, for particles between 37-149 $\mu \mathrm{m}$, the contaminant-surface interaction was the only significant interaction affecting the amount of contamination removed. The results obtained were already used at a contaminated site, confirming the prediction of contamination removed. 


\section{TABLE OF CONTENTS}

CHAPTER

PAGE

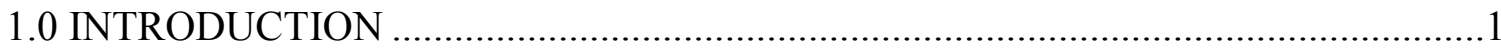

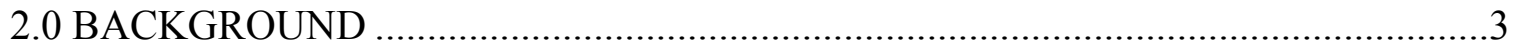

2.1 Chemical and physical interaction of atoms and molecules ............................... 3

2.2 Nature of attractive forces: Van der Waals forces ............................................... 4

2.2.1 Dipole-dipole interaction ................................................................ 4

2.2.2 Dipole-induced dipole interaction force ............................................... 5

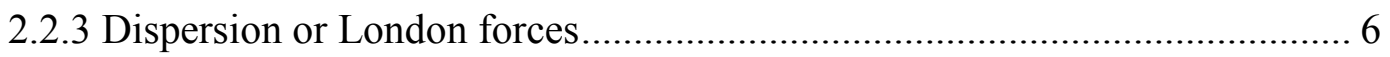

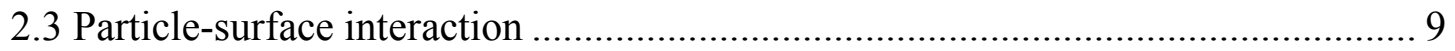

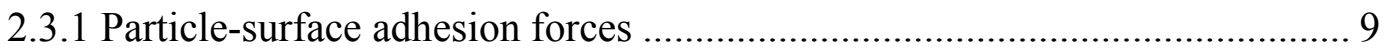

2.3.2 Mechanism of adhesion of a particle to a surface .................................... 12

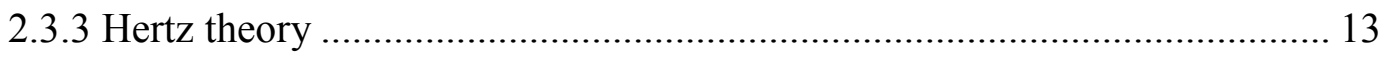

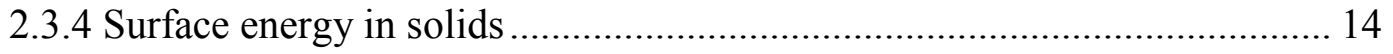

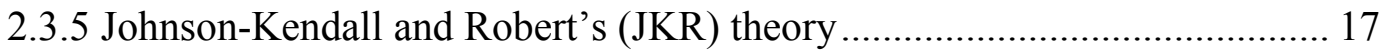

2.4 JKR theory application to predict scenarios of loose contamination removal on surfaces exposed to environmental conditions .................................................... 19

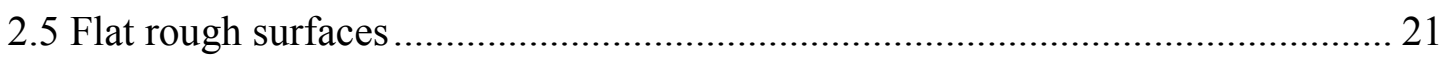

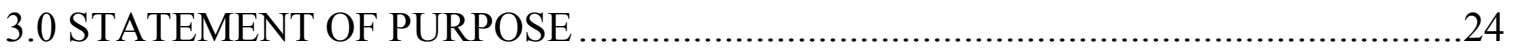

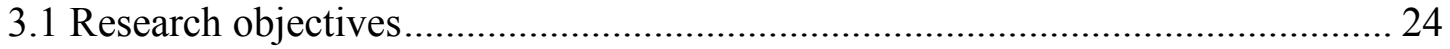

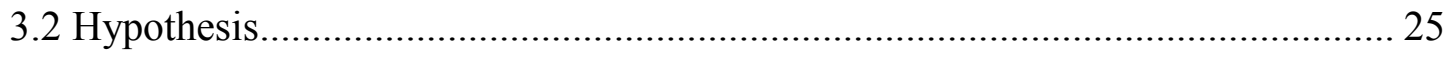

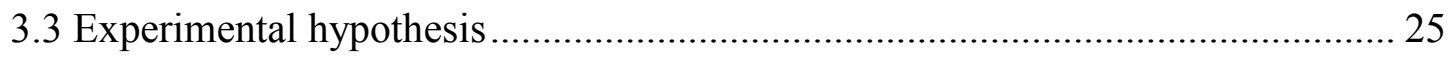

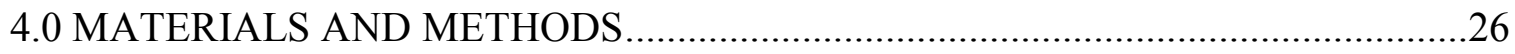

4.1 Method for characterization of the wipes ........................................................ 27

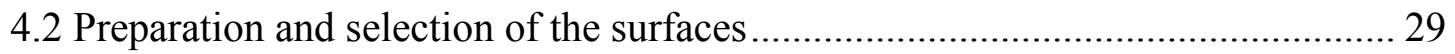

4.3 Method to quantify the contamination by weight ............................................ 31

4.3.1 Preparation and spread of the contaminant ........................................... 32

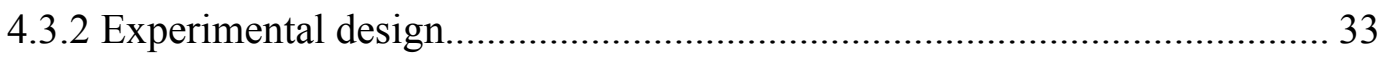

4.4 Method for quantification of the contamination removed using ${ }^{238} \mathrm{U}$ presented in uranium oxide powder as contaminant ............................................................ 34

4.4.1 Procedure to contaminate the surfaces with uranium oxide. ...................... 36

4.5 Method to quantify the contamination removed using Dowex 50Wx2 labeled

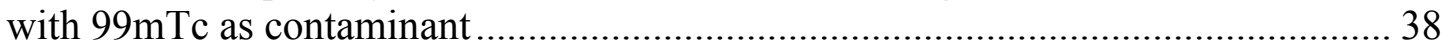


4.5.1 Method to label the Dowex 50Wx2 resin with ${ }^{99 \mathrm{~m}} \mathrm{Tc}$ : radioactive resin generation

4.5.2 Method to determine the appropriate ratio of concentration of $\mathrm{SnCl}_{2}$ to $\mathrm{TcO}_{4}{ }^{-}$ions to yield a high tag of ${ }^{99 \mathrm{~m}} \mathrm{Tc}$ to the cationic resin Dowex $50 \mathrm{~W} \times 2 \ldots . .41$

4.5.3 Particles preparation and characterization .............................................. 43

4.5.4 Approach to evaluate the efficiency of labeling of ${ }^{99 \mathrm{~m}} \mathrm{Tc}$ to resins of 200

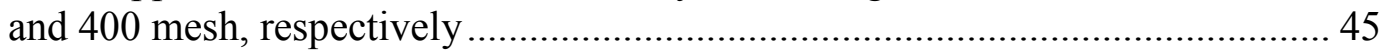

4.5.5 Method to evaluated the influence of ethanol in the ${ }^{99 \mathrm{~m}}$ Tc-resin bonding... 46 4.5.6 Method to determine the dispersion in number of counts of the contamination generated by the radioactive resin of 200 and 400 mesh

4.5.7 Method to optimize the sample/background ratio based on number of counts

4.5.8 Methods used to calculate the efficiency of loose contamination removed 51

4.5.9 Experimental design \& Model generation . 52

4.5.10 Contamination \& decontamination procedures followed to populate the

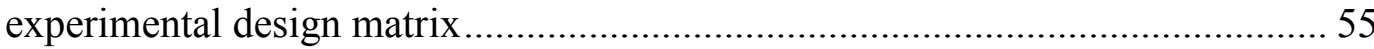

4.5.11 Instrumentation used to quantify the contamination removed from the surfaces 56

5.0 RESULTS .57

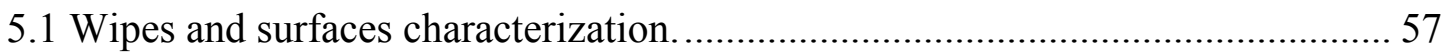

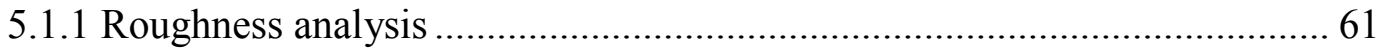

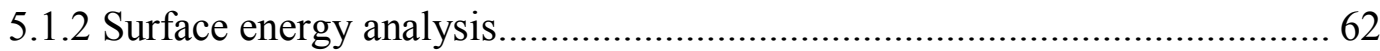

5.2 Quantification of the contamination by weighing procedures 64

5.3 Quantification of the contamination removed by radioactive detection, alpha disintegration mode....

5.4 Quantification of the contamination by radiation detection: gamma disintegration mode.

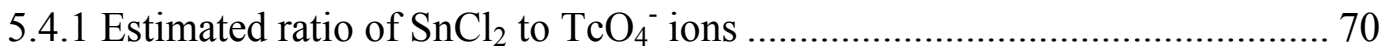

5.4.2 Estimated initial activity to obtain a good sample to noise ratio ................. 72

5.4.3 Estimated influence of ethanol in the ${ }^{99 \mathrm{~m}} \mathrm{Tc}$-Dowex $50 \mathrm{Wx} 2$ resin binding. 74

5.4.4 Roughness \& particle size influence on the efficiency of loose contamination removal: Model population \& data processing .......................... 75

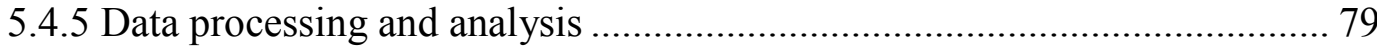

5.4.6 Surface energy \& particle size of the contaminant influence on the efficiency of loose contamination removal: Model population \& data processing 90

5.4.7 Surface energy data analysis.... 93 


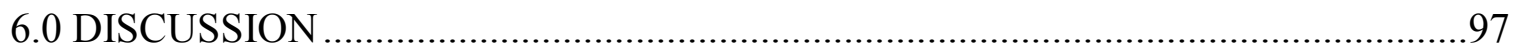

6.1 First scenario: Quantification of the contamination by weighing procedures .... 100

6.2 Second scenario: Quantification of the contamination by detection of alpha

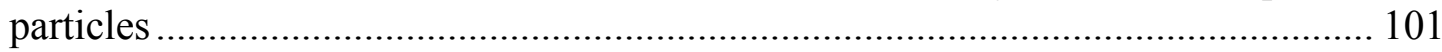

6.3 Third Scenario: Quantification of the contamination by detection of the

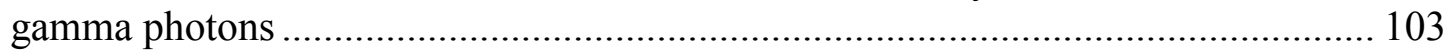

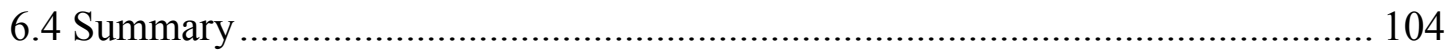

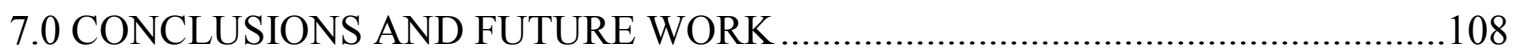

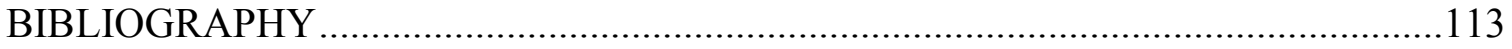

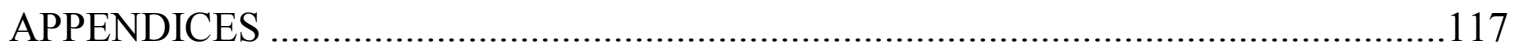




\section{LIST OF TABLES}

TABLE

PAGE

Table 1 Summary of Factors and Factors Levels..................................................... 33

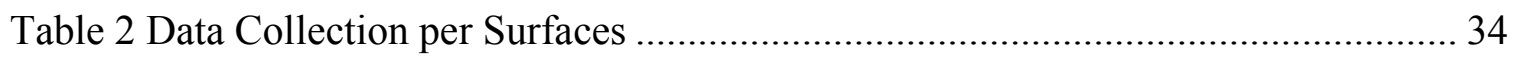

Table 3 Factor effect levels................................................................................... 54

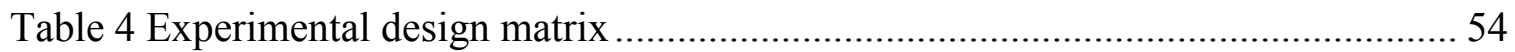

Table 5 Summary of the surface tension values of the residuals from wipe exposure..... 59

Table 6 Contact angle measurement for wipe 1,2 (cleaned), and $3 \ldots \ldots \ldots \ldots \ldots \ldots \ldots \ldots \ldots \ldots \ldots . . . . \ldots 0$

Table 7 Summary of roughness analysis made on the surfaces of concrete, PVC, tile

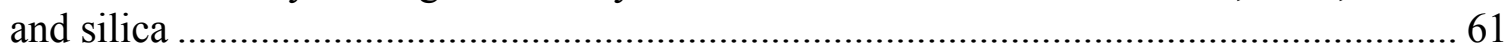

Table 8 Contact angle for ten random selected surfaces of PVC, silica, and concrete,

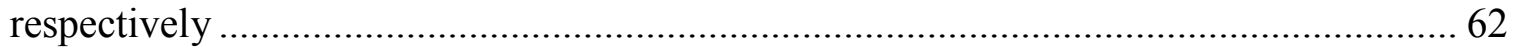

Table 9 Average and standard deviation in counts per minute of 22 surfaces, using the

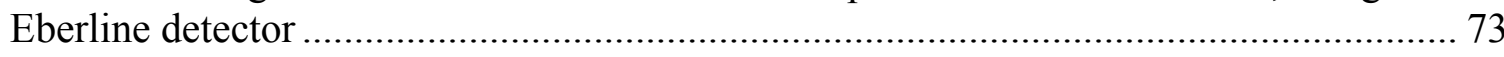

Table 10 Values obtained to calculated the efficiency of the detectors......................... 73

Table 11 Cobra Packard detector summary of the measurement ................................. 74

Table 12 Initial activity used for labeling the particles in a 200 and 400 mesh .............. 75

Table 13 Activities measured in the supernatant and the solid after labeling ................. 75

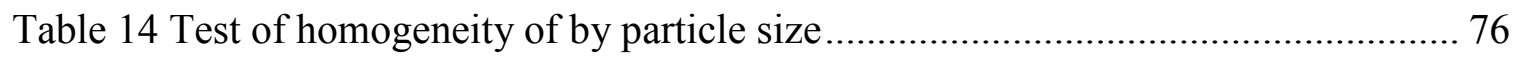

Table 15 Populated matrix with the experimental values obtained, following the order

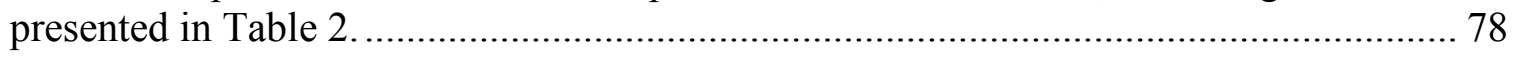

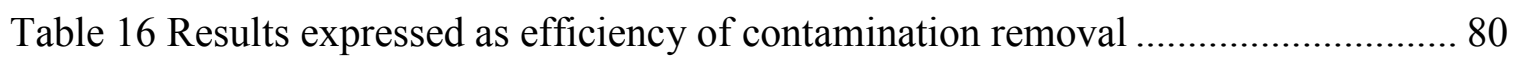

Table 17 Estimation of the amount of contamination per surface ................................ 80

Table 18 Data of the amount of loose contamination removal calculated using equation 2 .

Table 19 Estimated effect and coefficients of the factorial model for the Efficiency of Contamination Removal using data from Table 14 
Table 20 Analysis of Variance for the Efficiency of Contamination Removal obtained

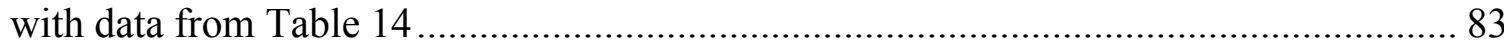

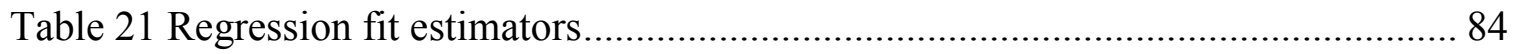

Table 22 Estimated effects and coefficients of the factorial model for the efficiency of

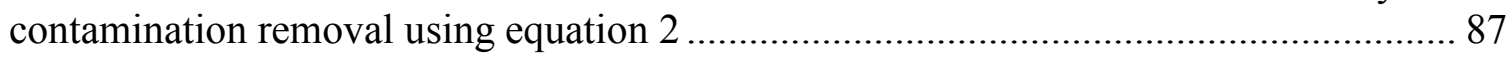

Table 23 Analysis of Variance for Efficiency of Contamination Removal obtained by

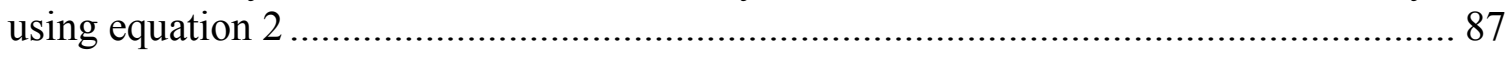

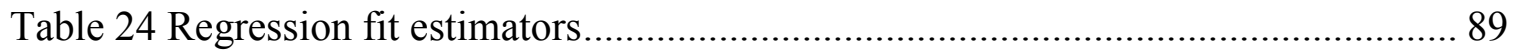

Table 25 Initial activity used for labeling the particles in a 200 and 400 mesh .............. 90

Table 26 Activities measured in the supernatant and the solid after labeling ................ 90

Table 27 Populated matrix with the experimental values obtained, following the order presented in Table 2 for surface energy considerations.............................................. 91

Table 28 Values of efficiency of removal of loose contamination for surface energy considerations

Table 29 Estimated effects and coefficients for observations presented in Table 20 ...... 93

Table 30 Summary of ANOVA table for surface energy considerations ....................... 94

Table 31 Regression fit estimators of the empirical model ........................................ 94

Table 32 Contamination detected during weekly survey at a Rad-Lab ....................... 110

Table 33 Data collected to obtain the amount of contamination removed by weighing

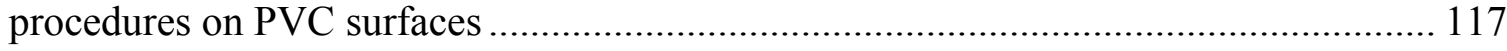

Table 34 Data collected to obtain the amount of contamination removed by weighing procedures on Formica surfaces.

Table 35 Data collected to obtain the amount of contamination removed by weighing procedures on ceramic surfaces.

Table 36 Table presenting the amount of uranium contamination removed from PVC surfaces

Table 37 Table presenting the amount of uranium contamination removed from

Ceramic surfaces.

Table 38 Table presenting the amount of uranium contamination removed from Concrete surfaces... 
Table 39 Table presenting the amount of uranium contamination removed from

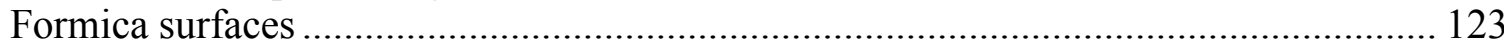

Table 40 Table presenting the amount of uranium contamination removed from Stainless Steel surfaces . 


\section{LIST OF FIGURES}

FIGURE

PAGE

Figure 1 Dipole-dipole interaction. In fixed dipoles (a) the interaction will depend on the angle between the two. In freely dipoles (b) the two might rotate to maximize the attractive forces or minimize the repulsion forces [2] ......

Figure 2 Induced dipole moment by an electric field. The atom has a symmetrically distribution of charges (a). An electric field of strength $E$ was applied and displaced the charge a distance $l[2]$.

Figure 3 London dispersion forces. The charges are symmetrically distributed within the atom or molecule (a). The charges are asymmetrically distributed and cause the dipole induction to the neighboring atoms, creating a coulombianan force of attraction between

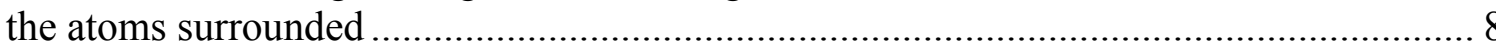

Figure 4 Free energy of attraction versus distance. The free energy becomes more negative (means that the forces of attraction will increase) until a limit in which the electron cloud of the atoms start to overlap each other. In this case the corn repulsion

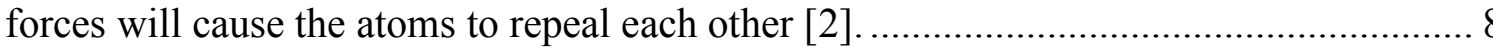

Figure 5 Gravitational, electrostatic, and van der Waals forces acting on a particle in

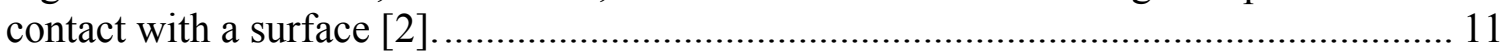

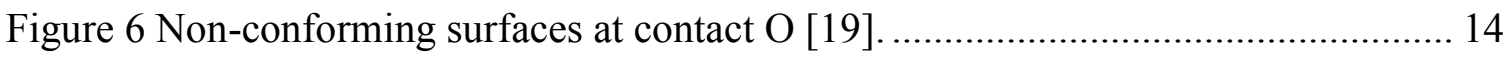

Figure 7 Force separation curve and surface energy for ideal surfaces [19] .................. 15

Figure 8 Surface free energy. a) Individual atom at bulk phase, b) atom at interface and

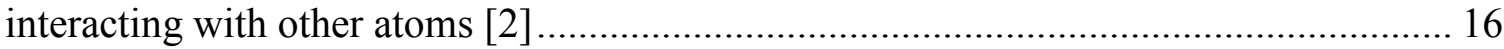

Figure 9 Separation of two identical surfaces to measure the surface energy ................. 17

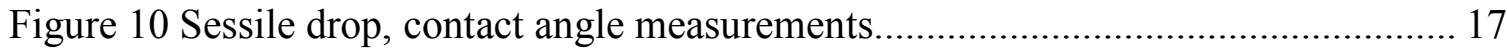

Figure 11 Drop Master equipment used to measure contact angle................................ 18

Figure 12 Contact area and surface roughness model, a) atomically smooth surface with a contact area that match the one predicted by the JKR theory, b) flat rough surface, the contact area is less than the one predicted by the JKR theory, c) flat surface with pronounced roughness that generates several points of contact. ................................... 20

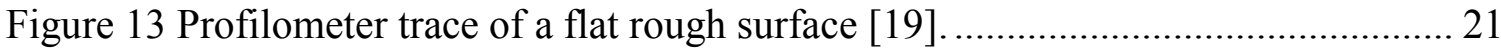

Figure 14 Flow chart predicting the amount of contamination removed........................ 23 
Figure 15 Wipes prepared to be studied under optical microscope

Figure 16 Fisher Semiautomatic Model 21 Tensiomat used to measured surface tension.....

Figure 17 Surfaces of PVC and silica while being contaminated with the radioactive resin 30

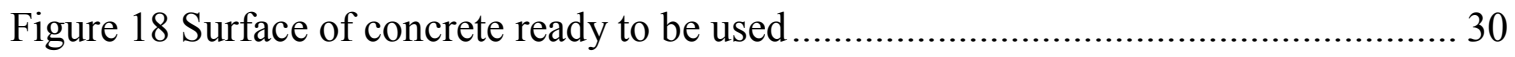

Figure 19 Drop Master Instrument, concrete surface under testing.............................. 31

Figure 20 Fluorescent powder in ethanol solution (left), image in a 100x

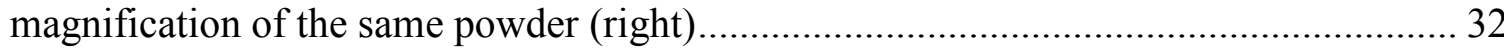

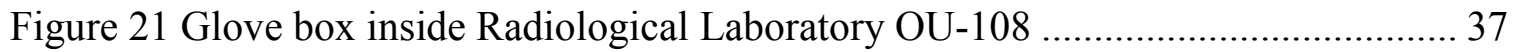

Figure 22 Wipe trajectory followed to decontaminated the surfaces ............................. 38

Figure 23 Experimental process of loose contamination generation. The resin was separated by particle size (74-149 $\mu \mathrm{m}$ and 37-74 $\mu \mathrm{m})$ and labeled with Tc-99m (items a. and b.). Then, it was shielded and spread in drops of $10 \mu \mathrm{l}$ on a selected

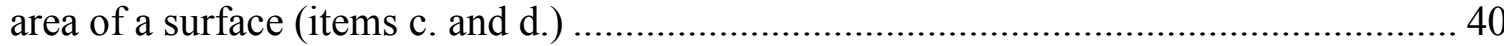

Figure 24 Summary of the resin labeling procedure.................................................. 42

Figure 25 Separation by weight of approximately one gram of resin (left side) and two centrifugation vials with $10 \mathrm{ml}$ of deionized water with one gram of 200 and 400 mesh resins in each, respectively (right side).

Figure 26 Resin of 200 mesh in deionized water after agitation (left side). Same resin after being centrifuged for $3 \mathrm{~min}$ at $4200 \mathrm{rpm}$

Figure 27 Flow chart followed to evaluate the efficiency of the labeling process of the

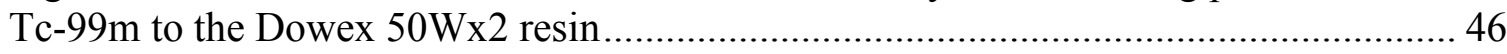

Figure 28 Test tubes used to check the variability in the mean number of counts of contamination of particles of $200(74-149 \mu \mathrm{m})$ and $400(37-74 \mu \mathrm{m}) \mathrm{mesh}$................... 48

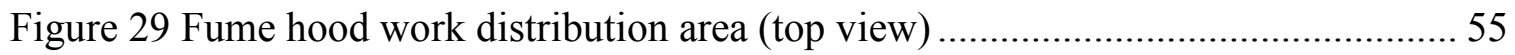

Figure 30 Eberline-E600 (left) and Cobra Packard II multisampling (right) detectors

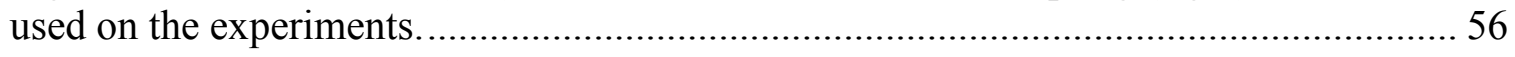

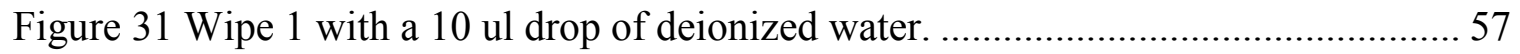


Figure 32 Wipe 1 and 2 (left and right, respectively) 100x magnification in presence of mounting oil

Figure 33 Wipes 3,1, and 2 submerged in water after being agitated with the sonicator for $10 \mathrm{~min}$ (left). After extracting the water from the wipes 2, 1 and 3 (right) ..... 58

Figure 34 Wipes 1,2 and 3 study under the Drop Master equipment. Wipe 1 and 3 upper part of the image, wipe 2 lower part.....

Figure 35 Atomic force microscopy of silica, PVC and concrete (left to right respectively)

Figure 36 Contact angle \& surface wetting behavior flow chart

Figure 37 Surfaces of PVC, silica, and concrete (left to right in that order) under analysis into the Drop Master equipment

Figure 38 Wipe 1 under optical microscope after being contaminated with the fluorescent powder. 65

Figure 39 Summary of the weighing procedure to estimate the contamination of surfaces. The data is presented as the mean amount of contamination removed \pm the standard deviation, both expressed in micrograms (Tables 33 through 35, appendix 9.1). 66

Figure 40 Chart of efficiency of contamination removal per type of wipe used and surface, respectively. The column bars represented the mean percentage of uranium oxide removed and the error bars the standard deviation (Tables 36 through 40, appendix 9.2).

Figure 41 Normal distribution plot of particles of 200 and 400 mesh (black and dash

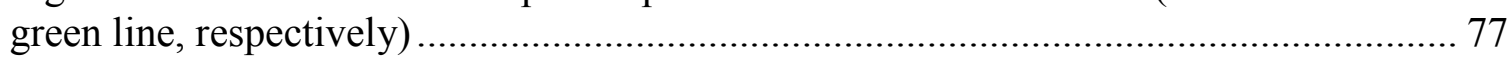

Figure 42 Pareto chart showing the significant effects within the empirical model......... 83

Figure 43 Contour plot of the Efficiency of loose contamination removal versus the wiped used, and the surface roughness for particles of 400 mesh... 85

Figure 44 Contour plot of the efficiency of loose contamination removal, particle size of the contaminant(y axis) versus roughness of the surfaces (x axis) for wipe 2 ...... 86

Figure 45 Pareto chart showing the significant effect within the empirical model .......... 88

Figure 46 Contour plot of the efficiency of loose contamination removal, particle size of the contaminant(y axis) versus roughness of the surfaces (x axis) for wipe 2 ...... 89

Figure 47 Contour plot of the efficiency of loose contamination removal, particle size of the contaminant(y axis) versus surface energy ( $\mathrm{x}$ axis) for both wipes 95 
Figure 48 Thermo-plastic on the Chicopee-Maslin microfibers viewed at $100 \mathrm{x}$ magnification ( 889 microns is the total length of the scale) 106

Figure 49 Dowex 50Wx2 resin on the Chicopee-Maslin microfibers viewed at $100 \mathrm{x}$ magnification ( 889 microns is the total length of the scale) ..................................... 107

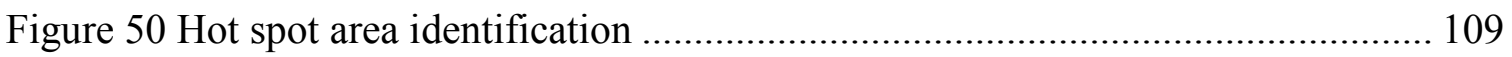

Figure 51 Area before and after decontamination efforts .................................... 111

Figure 53 Normal probability plot of the Residuals ................................................ 127

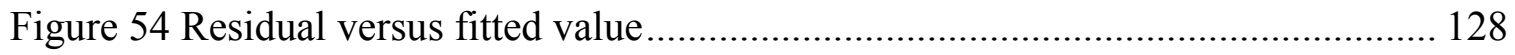

Figure 55 Residual versus particle size of the contaminant......................................... 128

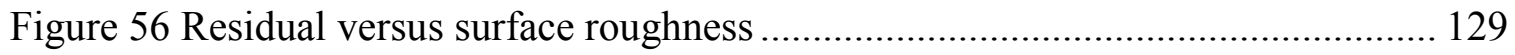

Figure 57 Normal probability plot of the residual ................................................. 130

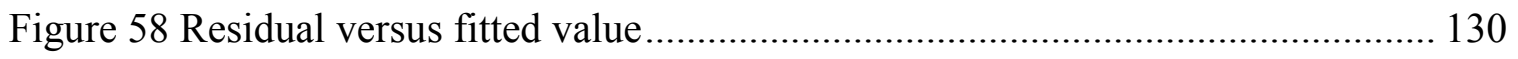

Figure 59 Residual versus particle size of the contaminant...................................... 131

Figure 60 Residual versus surface roughness .................................................. 131

Figure 61 Normal probability plot of the residuals.................................................. 134

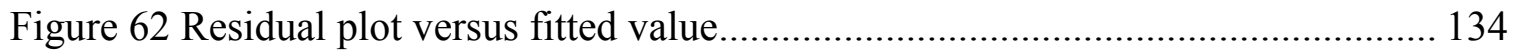

Figure 63 Residual versus particle size of the contaminant...................................... 135

Figure 64 Residual versus surface energy of the surface........................................ 135 


\subsection{INTRODUCTION}

Loose contamination on surfaces is an issue of remarkable significance in applications that range from mundane decontamination efforts (dust in clothes, on desks, in homes, etc.) to more esoteric ones (hazardous chemical dust generated by industrial processes, radioactive isotopes, etc.). The contaminants may be small particles, thin films, ionic compounds, molecular species, or microbiological agents. Small particles play a critical role because they can affect the performance of products such as hard drives, semiconductor wafers, etc. In addition, small particles can cause irreversible damage to human health, such as the case of workers directly exposed to contaminated areas or areas under decontamination efforts. The Occupational Safety and Health Administration (OSHA) estimated that 134,000 U.S. workers are potentially exposed to beryllium contamination (a form of loose contamination which causes a serious injury to human health known as "beryllium syndrome" [4]). Loose contamination might also be generated by unstable isotopes with different decays modes (alpha, beta or gamma), which constitute an external and internal exposure hazard to human health. On nuclear power plants, for example, during routine operations, items and materials used by the personnel in the plant have the potential of becoming slightly contaminated. Such low levels of contamination can be detected by daily smear surveys for evaluation of the removable or loose contamination and constitute a potential threat to the health of the employees of the plant.

The cleaning of small particles from surfaces is of vital interest to the members of society. Therefore, it is important to investigate the causes, the physical and chemical 
interaction that might exist and the best way to remove the contaminant from the surface, including an evaluation of the time of exposure to the contaminant and the effectiveness of the cleaning process.

In order to predict the desired level of cleanliness, it is necessary to address the principal forces and factors that affect the process of cleaning. From the literature $[1,2,3]$, the main forces that affect this process are the van der Waals forces (short range of action), electrostatic forces (longer range of action in comparison to the van der Waals forces), and capillary formation (in the presence of high specific humidity (greater than $70 \%$ [1])). These forces emerge from the physical phenomena of particle-particle interaction and particle-surface interaction. Nevertheless, intrinsic properties of the surfaces such as asperities, Young's modulus and Poisson's ratio of the contaminant and the surface, etc., can affect the extension and strength of their attractive forces. Also, the wipes involved in the cleaning process might have a pivotal role in obtaining the desired level of cleanliness in a short period of time. For example, most of the wipes on the market take advantage of the van der Waals interactions to remove loose contamination (microfiber cloths). Other wipes use the tribocharging effect (which is an electrostatic process) to capture dust and thus remove loose contamination from the surface. In addition, there are factors that will contribute to the extension and influence of these forces in the process of cleaning: the particle size of the contaminant, the surface roughness, and the type of wipe used in the cleaning process. The study of the interactions of all the factors mentioned will contribute to the assessment of the impact of the efficiency of removal of loose contaminants from surfaces. 


\subsection{BACKGROUND}

\subsection{Chemical and physical interaction of atoms and molecules}

From general chemistry, it is known that when two atoms bind to form a molecule, the force that keeps them together is a covalent force, and the binding is a covalent bond. These forces are classified as chemical forces; although they are strong in magnitude, they act over bond distance only, which means a short range of action (0.1-0.2nm) [2]. As a result, their study is limited to the atoms in the formation of molecules (intramolecular forces) or chemical reactions. On the other hand, the forces that characterize the interactions between molecules (intermolecular forces) are smaller in strength than the covalent forces but have a comparatively long range of action (10-1000nm) [2] . The forces that regulate this kind of interaction are classified as physical forces [1,2].

The fundamental forces that act over the physical interactions can be classified as electrostatic and van der Waals forces [2]. Although these forces are the main acting forces between particles, there are others forces that contribute to the phenomena of particle adhesion to surfaces as well. Examples include electrical double layer forces and the forces due to capillary formation $[1,3]$. The interactions that create the electrostatic forces are described by Coulomb's law and are present when two or more charged particles interact. However, the interactions that generate the van der Waals forces have their origin in electrodynamics theory, and are classified as dipole-dipole interactions, dipole-induced dipole interactions, and dispersion interactions. The last interaction is the origin of the so called "London or dispersion forces." 
In order to characterize the interaction that arises from the particles and the surfaces, it is necessary to address three important physical perspectives. First, the type of physical forces involved in the process of interaction between molecules and molecules, and molecules and non-associated atoms. Second, the interactions caused between the molecules or atoms with the surface. Finally, the mechanical stress caused by the interaction of the particle with the surface.

\subsection{Nature of attractive forces: Van der Waals forces}

The van der Waals forces are characterized by three different types of interaction that generates the basic nature of the attraction between particles [3]. The first two interactions (dipole-dipole and dipole-induced dipole) are the strongest of the three. However, the dispersion forces are the more universal and are often the more important contributor to the van der Waals forces [2].

\subsubsection{Dipole-dipole interaction}

The dipole moment is a result of an asymmetric distribution of the cloud of electron charge in a molecule [2]. Although atoms in nature generally have a symmetrical distribution of electronic charge, under the presence of an electric field or a strong polar molecule, their charge distribution will not be symmetrical to the nucleus, then creating a dipolar moment.

When two polar molecules with dipole moments $\mu_{1}$ and $\mu_{2}$ approach each other in the presence of a vacuum, there will be a dipole-dipole interaction which will cause a resultant attraction or repulsion force in dependence of the orientation of the dipoles. Of 
course, if the dipoles cannot freely move, they can rotate and orientate in a configuration in which the attraction forces surpass the repulsion forces (Figure 1)[2].

The dipole-dipole interactions in real life are not very significant unless very small molecules with a very large dipole moment are interacting, which is the case with water, hydrogen fluoride, or ammonia. If that is the case, a new interaction occurs, namely, a hydrogen bond. In such cases, as the hydrogen atoms are very small, more electronegative atoms will approach very close to the hydrogen atoms, creating a very strong electric interaction, which increases the attraction forces.

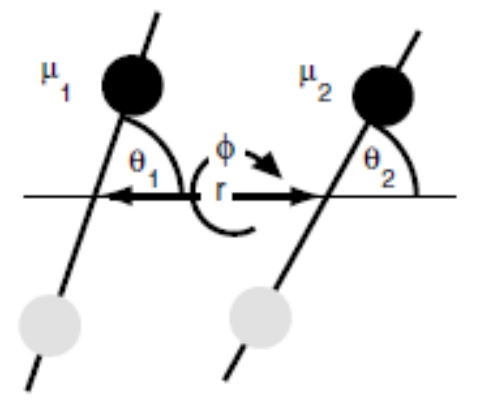

(a)

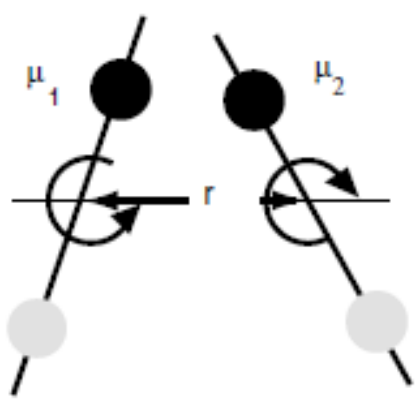

(b)

Figure 1 Dipole-dipole interaction. In fixed dipoles (a) the interaction will depend on the angle between the two. In freely dipoles (b) the two might rotate to maximize the attractive forces or minimize the repulsion forces [2]

\subsubsection{Dipole-induced dipole interaction force}

The polarization of an atom or molecule is caused by the presence of an electric field or a polar molecule that can displace the electronic charge of the first to a distance $l$ (Figure 2) [2].

The force generated by the dipole-induced dipole interaction is caused when a strong polar molecule approaches a non-polar molecule. This process is similar to the dipole 
induced by an ion to a non-polar molecule. The difference is that the ion will use an electric field to induce the dipolar moment and the polar molecule will use a strong dipolar moment.

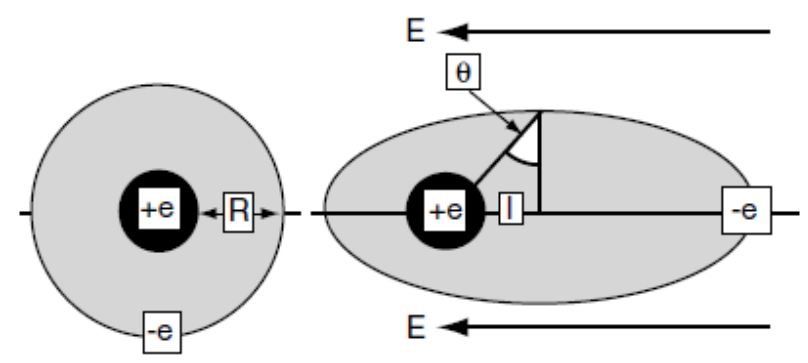

(a)

(b)

Figure 2 Induced dipole moment by an electric field. The atom has a symmetrically distribution of charges (a). An electric field of strength $E$ was applied and displaced the charge a distance $l[2]$

\subsubsection{Dispersion or London forces}

The dispersion forces, even when they are the weakest forces among the three components of the van der Waals forces, are the more universal, as compared to the dipole-dipole or dipole induced dipole forces, which varies with the chemical nature of the species.

The more important characteristics of the dispersion forces are:

- They have more range of action than the covalent forces

- They may cause attraction or repulsion, depending on the situation

- They are non-additive, which means that the interaction between atoms or molecules will be affected by the nearby atom or molecule interaction. 
The dispersion force is mainly a probabilistic phenomena explained by the quantum mechanics theory. It involves interactions between sudden fluctuations of dipoles as a result of movement of the outer valence electrons of an atom or molecule. As an example, in a system of two isolated atoms or molecules, the cloud of electrons can move around the atom or molecule symmetrically to the core (Figure 3a). However, they will not always be at the same distance from the nucleus or will keep the same symmetry of charge distribution, thus creating some asymmetry distribution of charges (Figure 3b). This generates an ephemeral electric field which it is caused by a shift of dipoles originated during this process. This phenomenon causes the polarization and induction of a dipole to the neighboring atoms of molecules. As a result, a net Coulombic attraction between the two starts to appear. These interactions can be considered electrostatic interactions (Figure3b).

A graph of the free energy of attraction as a function of the distance of two atoms or molecules is represented in figure 4 [2]. From Figure 4, it can be inferred that, even when the attraction forces increase at shortened distances, there is a threshold at which the two atoms or molecules get too close and their electron clouds screen (from the nucleus or positive charge) each other and create a resultant repulsion force between them, known as Born repulsion.

All these assumptions were made in the presence of a vacuum. In reality, the medium affects the contribution of the van der Waals forces between atoms or molecules. Particularly, the dispersion forces can take an attractive or repulsive character, depending on the ionization potential of the medium involved. In addition, the van der Waals forces 
have a non additive character and the magnitude of the force is affected by neighboring interacting phenomena.

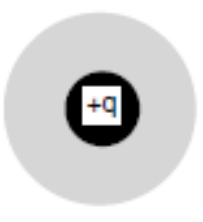

(a)

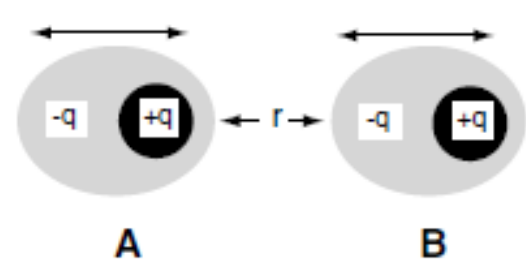

(b)

Figure 3 London dispersion forces. The charges are symmetrically distributed within the atom or molecule (a). The charges are asymmetrically distributed and cause the dipole induction to the neighboring atoms, creating a coulombianan force of attraction between the atoms surrounded

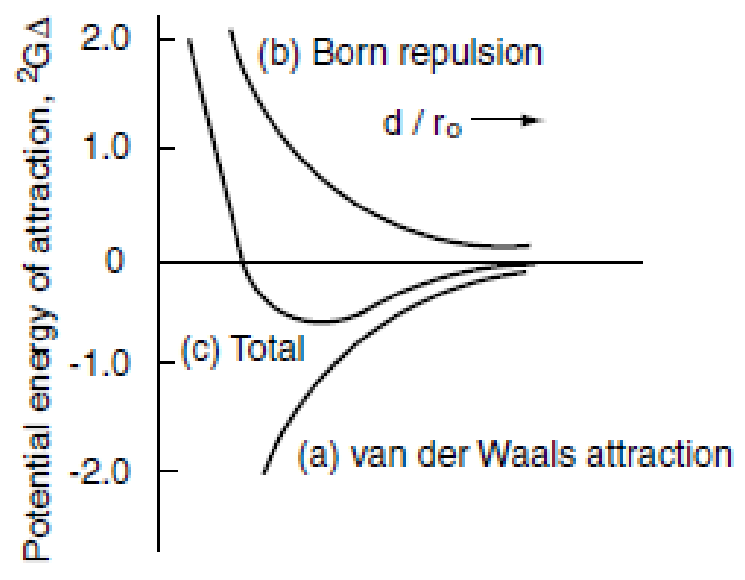

Figure 4 Free energy of attraction versus distance. The free energy becomes more negative (means that the forces of attraction will increase) until a limit in which the electron cloud of the atoms start to overlap each other. In this case the corn repulsion forces will cause the atoms to repeal each other [2].

For example, when two atoms are interacting in a system composed of several of atoms, the first two will feel a reflection of the interaction of their neighbors which will contribute to an increase or decrease in the magnitude of their interaction. In addition, the influence of the medium has an anisotropic nature. This means that the ability of 
molecules to polarize depends on their orientation. This effect has the most influence in the case where molecules are not free to move, such is the case for crystal, polar liquids, solids, etc.

Summarizing, the theory of the van der Waals forces has its limitations, especially when very strong polar molecules are involved in the process of cleaning. For example, the force of attraction predicted by the van der Waals theory of two strong polar molecules is bigger than the real force seen in the experimental process. This means that polar molecules would rather interact with molecules of their own kind, and then create a separation between the two different types of molecules (polar and non-polar). Such is the case for vegetable oil and water, or methane molecules and water molecules [2]. Conventionally, this mutual dislike or lack of interaction is known as hydrophobic behavior, which means fear of water. On the other side, when polar molecules interact with water (can be a molecule less polar than molecules of water), the phenomena is known as hydrophilic behavior or affinity for water.

\subsection{Particle-surface interaction}

\subsubsection{Particle-surface adhesion forces}

Forces in nature can be classified as one of four types: strong interactions, weak interactions, electromagnetic interactions and gravitational interactions [3]. When a solid particle contacts a surface or other solid particle, the primary force between these solids is of attraction [3]. The resultant force of adhesion will have components of all the forces

due to the interactions mentioned above. Nevertheless, the diameter of the particle and 
the distance of the particle from the surface play a critical role in the strength and contribution of each component of the force to the total adhesion force.

In the case of macroscopic particles, the major component of the adhesion forces is the gravitational force (Figure 5). However, as the diameter of the particle decreases, the gravitational forces start losing strength proportional to the third power of the size of the particle's radii. In consequence, adhesion force components might vary in strength and significance. From Figure 5, it can be observed that, for particles with radii less than $0.0015 \mathrm{~m}$, the van der Waals and electrostatic forces are the dominant components of the adhesion forces.

Electrostatic interactions are involved in the phenomena in which a net displacement of charges occurs, which means a formation of a dipole moment. In general, when a charged particle approaches a substrate, an attractive electrostatic force is formed even if the substrate is a conductor or a dielectric [3]. In general, electrostatic forces decrease with the particle diameter.

In comparison with the gravitational force, the electrostatic forces decrease slower than the gravitational forces with the radii of the particle. Also, for smaller particles, the component of the electrostatic forces is greater than the component of the gravitational forces. Of course, going to the other side, the role of the gravitational forces is greatest as the particle acquires macroscopic sizes. 
Finally, the forces of adhesion that have an electrodynamics origin are the van der Waals forces. The first van der Waals interaction is caused by the free rotation of permanent dipoles to align each other, known as Keesom interactions

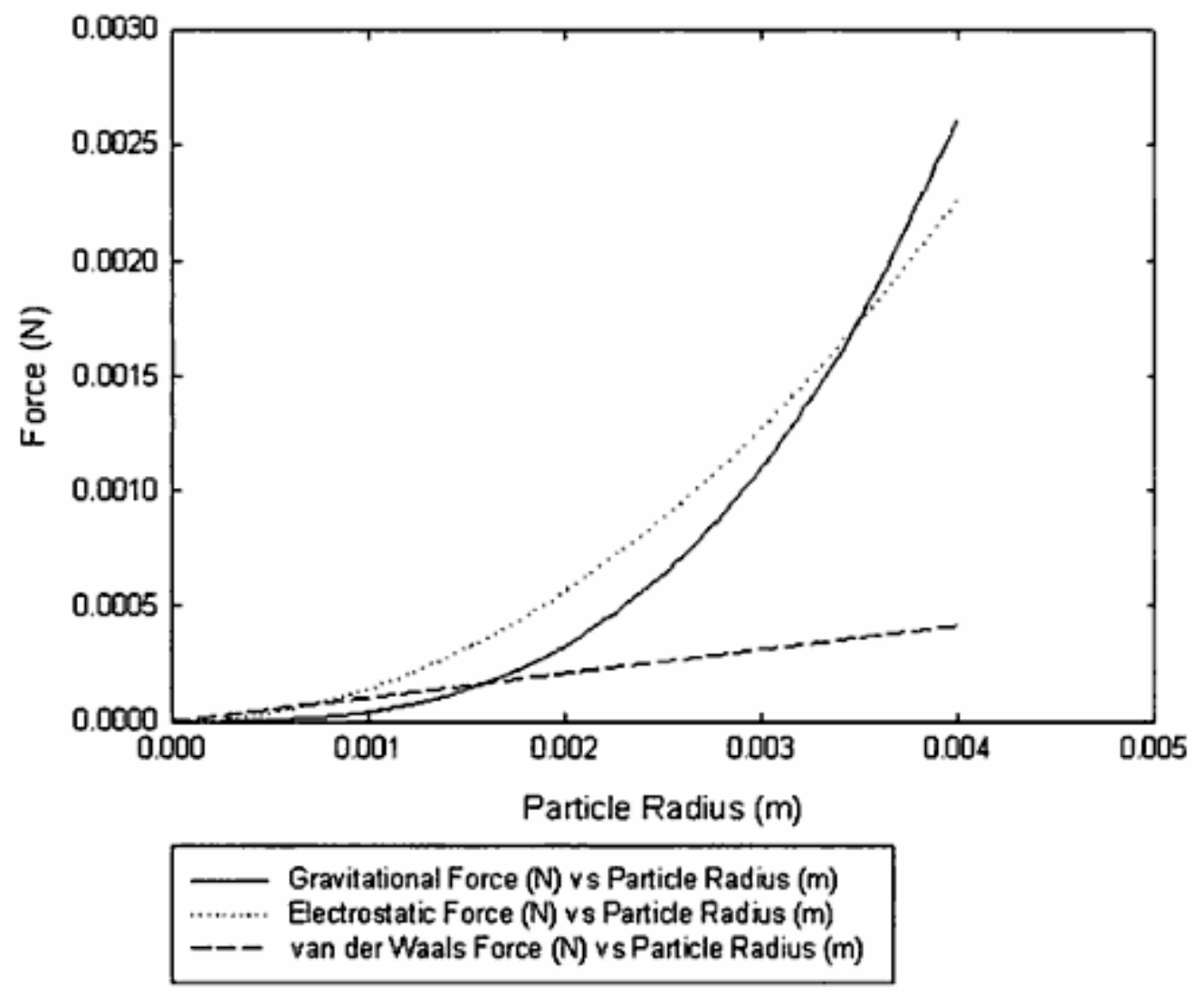

Figure 5 Gravitational, electrostatic, and van der Waals forces acting on a particle in contact with a surface [2].

The second interaction is caused by the induction and aligning of the dipole and orientation of neighbor molecules, respectively (Debye interactions). The third interaction comes when instantaneous dipoles induce instantaneous dipole in the neighboring atoms or molecules (London or dispersion forces). Van der Waals forces increase linearly with the radius of the particle. As well as the gravitational and the electrostatic forces, the three forces increase with the particle diameter. Nevertheless, the 
increase of the van der Waals and electrostatic forces are slower than the increase of the gravitational force, which means that for particles with small radii (going from micron to nano radii), the component of the adhesion forces that will dominate the attraction between the particle and the surface is the component of the van der Waals and electrostatic forces. On the contrary, for particles with big radii (going from nano to micron or milli radii) the gravitational force will be the dominant component of the adhesion forces.

As can be seen, all the forces depend directly on the diameter of the particle. However, practice has shown that the mechanical forces required to remove dry particles from a surface should be proportional to the third power of the diameter of the particle (in case of centrifugal forces) and proportional to the second power of the diameter of the particle (in case of using a force generated by air flow).

\subsubsection{Mechanism of adhesion of a particle to a surface}

The previous sections presented the interactions between particles-particles and surfaceparticles. Nevertheless, the mechanical responses (stress) generated due to the contact of a particle with a surface also influences the strength by which the particle is adhered to the surface. Besides, the magnitude of the force with which a particle adheres to a surface is not the same as the force necessary to remove it. Actually, experiments confirm that when the diameter of the particles decreases, the mechanical stress necessary to remove that particle from the surface decreases in a proportion to a third power of the particle diameter [1]. 
The mechanical properties of the materials involved in the contaminated area (such as young modulus, Poisson ratio, etc.) play an important role in predicting the removal of particles from a surface. The integration of the adhesion forces with the mechanical responses of the interacting materials helps to predict how strong a particle is attached to a substrate and, hence, how easily it can be removed. Those studies are part of the branch of physics know as contact mechanics, which is the study of the deformation of solids that contact each other at one or several points.

\subsubsection{Hertz theory}

Heinrich Hertz developed the first theory related to this subject as early as 1880 . This theory is known as the "Hertzian Theory of Elastic Deformation," and it predicts the contact area of two interacting bodies based on their elastic deformation due to their physical composition.

The Hertz theory considers two elastic spheres in contact (know as non-conforming solids, see Figure $6[19])$ at one point or in a line of contact. The theory predicts the deformation of the spheres in the vicinity of the point of contact by a normal load applied.

The Hertzian theory relates the area of contact of a particle with a surface based on the combined effect of their elastic properties, the load applied and the particle radii. However, experiments have shown that when the load is removed, there is still an adhesion load remaining that attaches the particle to the surface [19]. This adhesion load is due to the influence of the adhesion forces close to the region of contact. 
As a result, the two bodies in contact will have an equilibrium distance known as $z_{0}$. At separation below $z_{0}$, the two bodies will repel each other; at separations greater than $z_{0}$, they will attract (Figure 7).

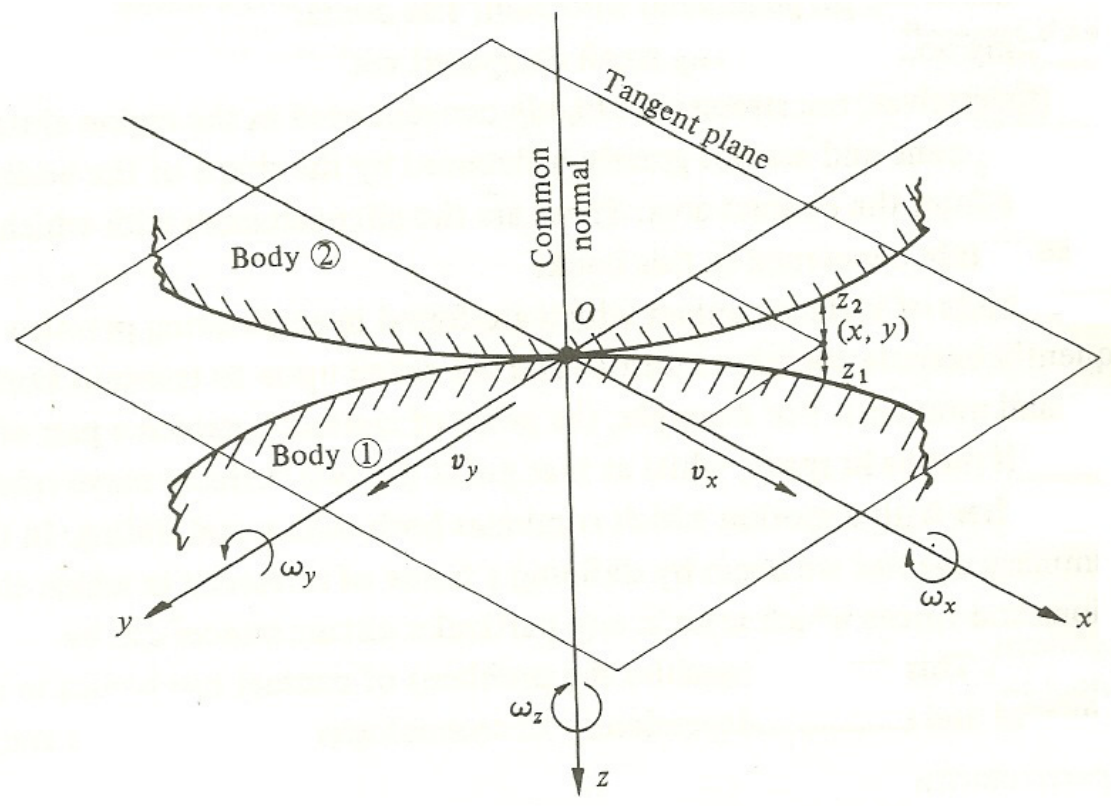

Figure 6 Non-conforming surfaces at contact O [19].

The surface's forces of solids are very hard to measure [19]. Also, it is easier to measure the work necessary to split apart the particle and the surface from the distance $\mathrm{z}=\mathrm{Z}_{\mathrm{o}}$ to $z \rightarrow \infty$. This work will depend on the surface energy of each solid interacting, which depends also on the solid's physical composition.

\subsubsection{Surface energy in solids}

Surface energy quantifies the disruption of intermolecular bonds that occurs when a surface is created. If the atoms or molecules of a surface are on their bulk phase (Figure 8a), the force field will be distributed uniformly through the bulk and the net resultant attractive force is zero. But at the interface, the forces between atoms or molecules 
increase (generally) and a net attraction force is created instead (Figure 8b). As a result, an excess of energy is created, which is known as surface energy or surface free energy.

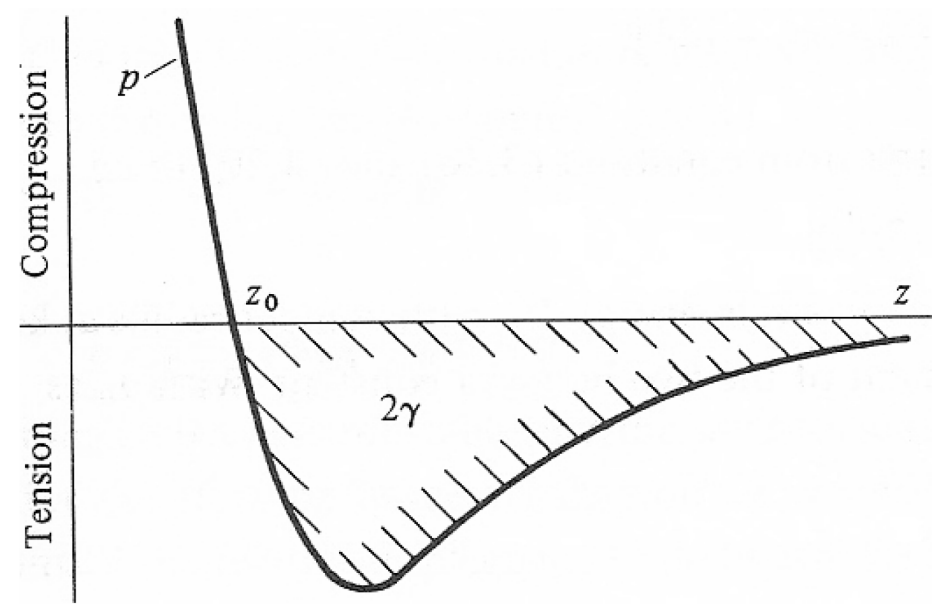

Figure 7 Force separation curve and surface energy for ideal surfaces [19]

There are mainly two ways by which the surface energy can be obtained: the first by measuring the cohesion of solids, or the second, by studying the wetting behavior of a range of liquids with different surface tensions on the solid surface [2]. However, neither of the methods is straight forward. The cohesive energy of two solids is equal to the energy necessary to separate those solids (Figure 9) but, after the separation, irregularities appear on the new surfaces. Hence, the true area is greater than the geometric area. Although under controlled conditions some materials can be studied this way, such as layered natural aluminosilicate crystal and muscovite mica [2], the estimation of the surface energy for the rest of the materials needs to be approached in a different way.

The second method has the advantage that it is more general but is still very specific to one type of surface. In addition, the number of liquids with different surface tensions is huge, which creates a disadvantage. This method uses the contact angle of a drop of 
liquid (Figure 10) to estimate the surface energy of the surface by using the Young's equation expressed as [2]:

$$
\gamma_{S V}=\gamma_{S L}+\gamma_{L V} \cos \theta
$$

Where: $\gamma_{S V}, \gamma_{S l}$,and $\gamma_{L V}$ are the energy at the solid-vapor, solid-liquid, and liquid-vapor interface, respectively.

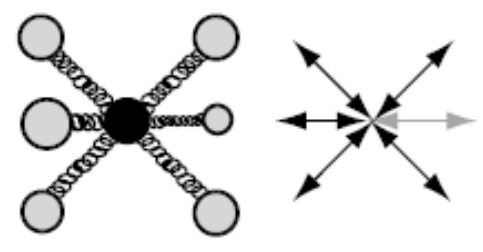

Bulk atom: net force $=0$.

a)

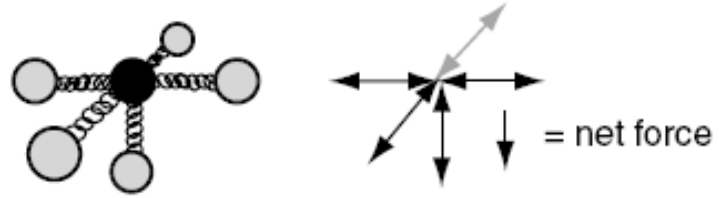

Surface atom: net attraction of surface atom into the bulk phase.

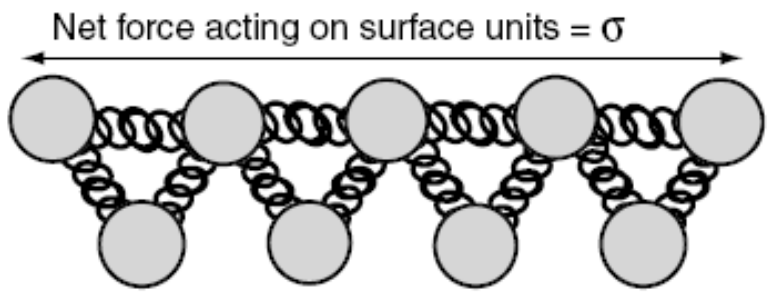

Figure 8 Surface free energy. a) Individual atom at bulk phase, b) atom at interface and interacting with other atoms [2]

The method takes liquids with different surface tensions and measures the theta angle on the surface (Figure 10); when a liquid with a known surface tension is found that makes $\theta=0$ (complete wetting case), the value of $\gamma_{S L}$ approaches zero [2], and the surface tension of the liquid is approximately equal to the surface energy of the solid (see Young's equation above). The contact angle can be measured experimentally by a Drop Master equipment which measures the contact angle of a drop for liquids of different surface tensions (Figure 11). 


\subsubsection{Johnson-Kendall and Robert's (JKR) theory}

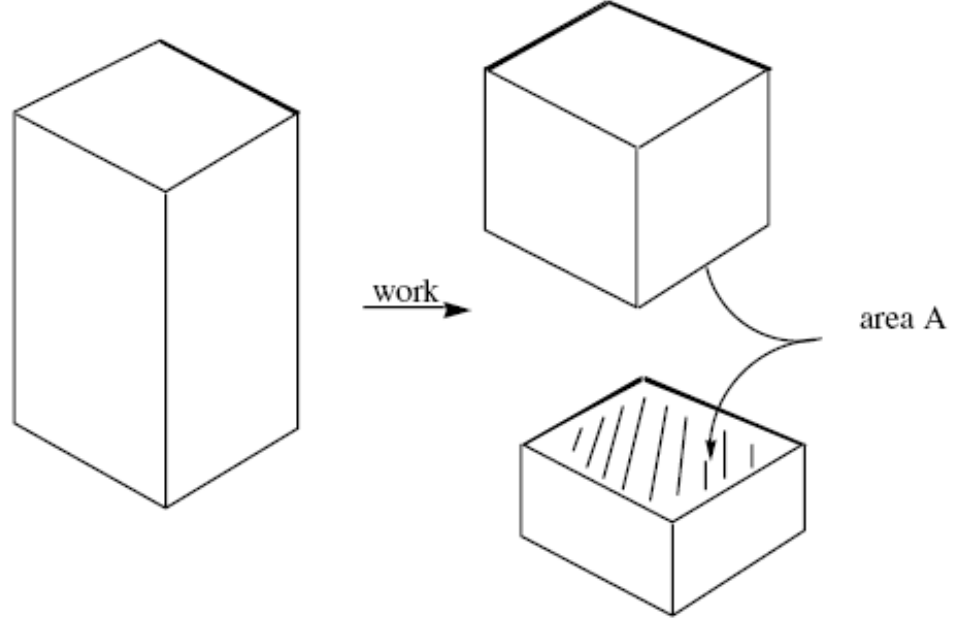

Figure 9 Separation of two identical surfaces to measure the surface energy

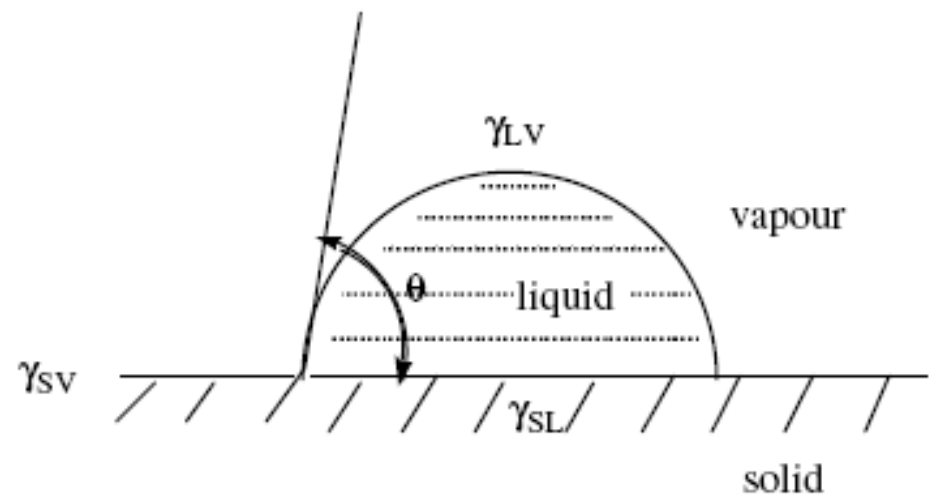

Figure 10 Sessile drop, contact angle measurements

As explained, the Hertz's theory does not consider the existence of the adhesion forces (van der Waals forces, electromagnetic forces, etc.) in the region of contact between the two surfaces. Nevertheless, the Hertzian theory was the starting point of further analysis carried out by Johnson, Kendall and Robert, in which the influence of these forces to the contact area are considered. This theory is known as the JKR theory. 
The JKR theory predicts the area of contact between a particle and a surface based on their adhesion force at the interfacial region, and the particle radii as:

$$
a^{3}=\frac{3 R}{4 E}\left(P+3 \pi w_{a} R+\sqrt{6 \pi R w_{a} P+\left(3 \pi R w_{a}\right)^{2}}\right)
$$

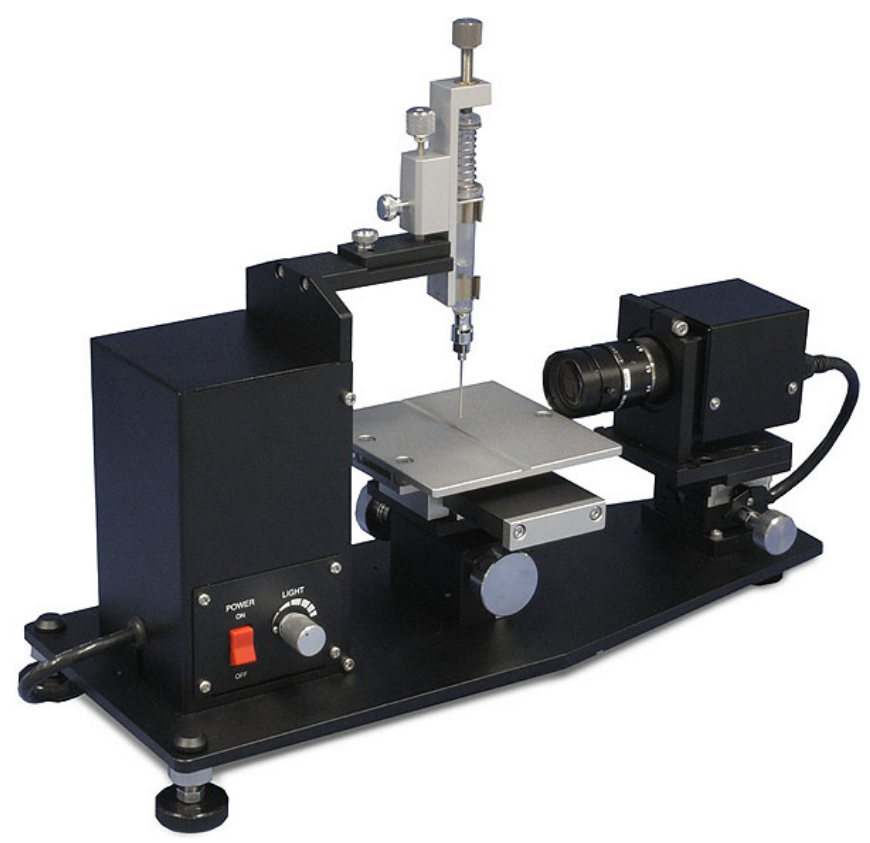

Figure 11 Drop Master equipment used to measure contact angle.

Equation 2 is known as the JKR equation. From equation 2, the force necessary to detach a particle from a surface can be found. This force is reached when the radii of contact vanish or when it is close to zero. Equation 2 has a constraint that can be expressed as:

$$
6 \pi R w_{a} P+\left(3 \pi R w_{a}\right)^{2} \geq 0
$$

If this condition is violated, then the radii of contact will have an imaginary value which, in practical terms, is impossible. In consequence, a threshold of applied load can be found by which the radii of contact is real (load not enough to detach the particle from the surface) and by which the radii of contact is imaginary (the particle has been removed 
from the surface). This threshold can be found as a result of equation 3 and is expressed as:

$$
P_{\text {detach }} \geq \frac{3}{2} w_{a} \pi R
$$

Equation 4 relates the detachment load necessary to remove a particle from a surface with the particle radii and with the physical properties of the particle and the surface.

The work of adhesion $\left(\mathrm{w}_{\mathrm{a}}\right)$ is defined as the reversible work necessary to separate a unit area of two identical surfaces with a surface energy $(\gamma)$ [1]. When the composition of the particle and the surface are different, the work of adhesion is defined as the reversible work necessary to separate a unit area of two different materials (1 and 2) [1], and is defined as:

$W_{a}=\gamma_{1}+\gamma_{2}-\gamma_{12}$

Where $\gamma_{1}, \gamma_{2}$ are the surface energies of the materials 1 and 2, respectively, and $\gamma_{12}$ is the interfacial energy between the two materials.

\subsection{JKR theory application to predict scenarios of loose contamination removal on surfaces exposed to environmental conditions}

In practice, experiments have shown that adhesion between solids is not easily observed nor measured [19]. The reason for this lack of visible adhesion is the surface roughness of the interacting bodies. In the case of small contaminants on large surfaces, the roughness

of the surface is a factor that it needs to be taken into consideration. Actually, the area of contact predicted by equation 2 does not account for this phenomena, and in presence of 
roughness, the observed area of contact is smaller than the one predicted by the JKR equation (Figure 12).

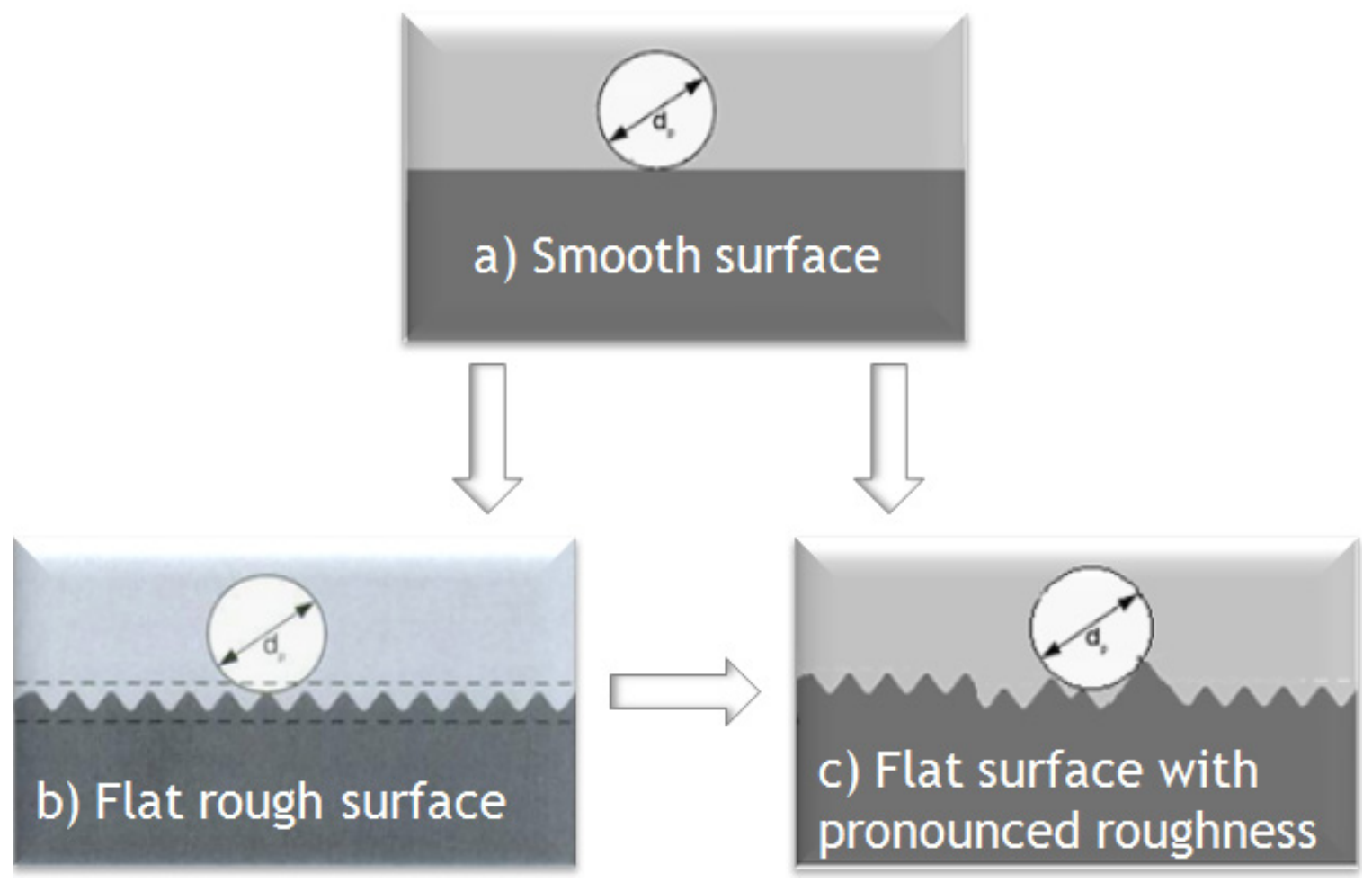

Figure 12 Contact area and surface roughness model, a) atomically smooth surface with a contact area that match the one predicted by the JKR theory, b) flat rough surface, the contact area is less than the one predicted by the JKR theory, c) flat surface with pronounced roughness that generates several points of contact.

The JKR theory provides an equation (equation 4) to predict the detachment force to remove a contaminant from the surface based on their physical and chemical characteristics, but this equation does not provide information about the amount of contamination removed from an area that is under decontamination efforts nor predicts the amount of contamination removed based on the roughness of the surface. 


\subsection{Flat rough surfaces}

The effect of surface roughness (Figure 12 items b. and c.) creates a discontinuous contact between the contaminant and the surface. Also, roughness in "real" surfaces (surfaces that are not treated for special conditions) are not regular. Thereby, the heights of the asperities vary in a randomly pattern (Figure 13).

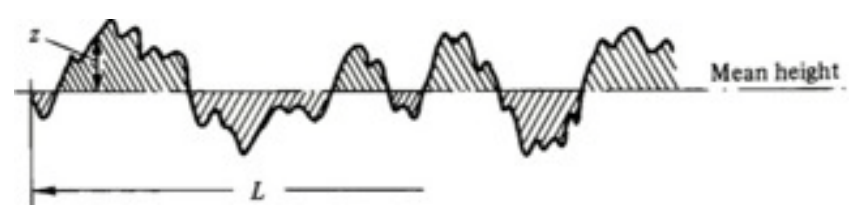

Figure 13 Profilometer trace of a flat rough surface [19].

From Figure 12, the followings conditions can be obtained assuming that the particles are spheres and are perfectly smooth:

1. If the surface roughness is a lot less than the radii of contact $\left(\mathrm{R}_{\mathrm{a}}<<\mathrm{a}\right)$ then the surface can be treated as a perfectly smooth surface (Figure 12a),

2. If the surface roughness is less than the radii of contact $\left(\mathrm{R}_{\mathrm{a}}<\mathrm{a}\right)$, then the surface can be treated as a flat rough surface (Figure 12b),

3. If the surface roughness is greater than the radii of contact $\left(R_{a}>a\right)$ but less than the particle radii $\left(\mathrm{R}_{\mathrm{a}}<\mathrm{R}_{\mathrm{p}}\right)$ then the surface is a flat rough surface in which several contact points might occur (Figure 12c).

The conditions mentioned above (Figure $12 \mathrm{~b}$ and 12c) are presented in surfaces more likely to be exposed to environmental conditions, in which oxidization, agglomeration of particles, surface inhomogeneities, etc., can be observed regularly. The study of the combined effect of the radii of the contaminant and the roughness of the surface is a 
phenomenon that severely affects the detachment force of a contaminant to a surface, and is not describe by the Hertz, JKR or any theory developed so far. This is why it is very important to address this issue in order to predict scenarios of removal of loose contaminants from surfaces. It is necessary to find an empirical model able to predict not the detachment force but the amount of contamination removed from surfaces that are under decontamination efforts.

In addition, in real life scenarios, decontamination of surfaces is carried out by wiping procedures. Thus, it is necessary to add the wipe-particle interaction in the decontamination effort. For example, most of the wipes take advantages of the van der Waals interactions to remove contamination (microfiber cloth). The extension of the particle-wipe interaction can be quantified by taking comparative values of the amount of particles removed. This magnitude can be estimated by the efficiency of removal of loose contamination and relates the contamination that remains on the surface before and after decontamination by wiping procedures, and can be used to estimate the influence of the particle-surface interaction and particle-wipe interaction on the decontamination procedures.

Finally, the amount of loose contamination removed by cleaning a surface depends on the surface roughness, the particle size of the contaminant, and the physical characteristics of the contaminant and the surface, represented by their surface energy values (Figure 14). 


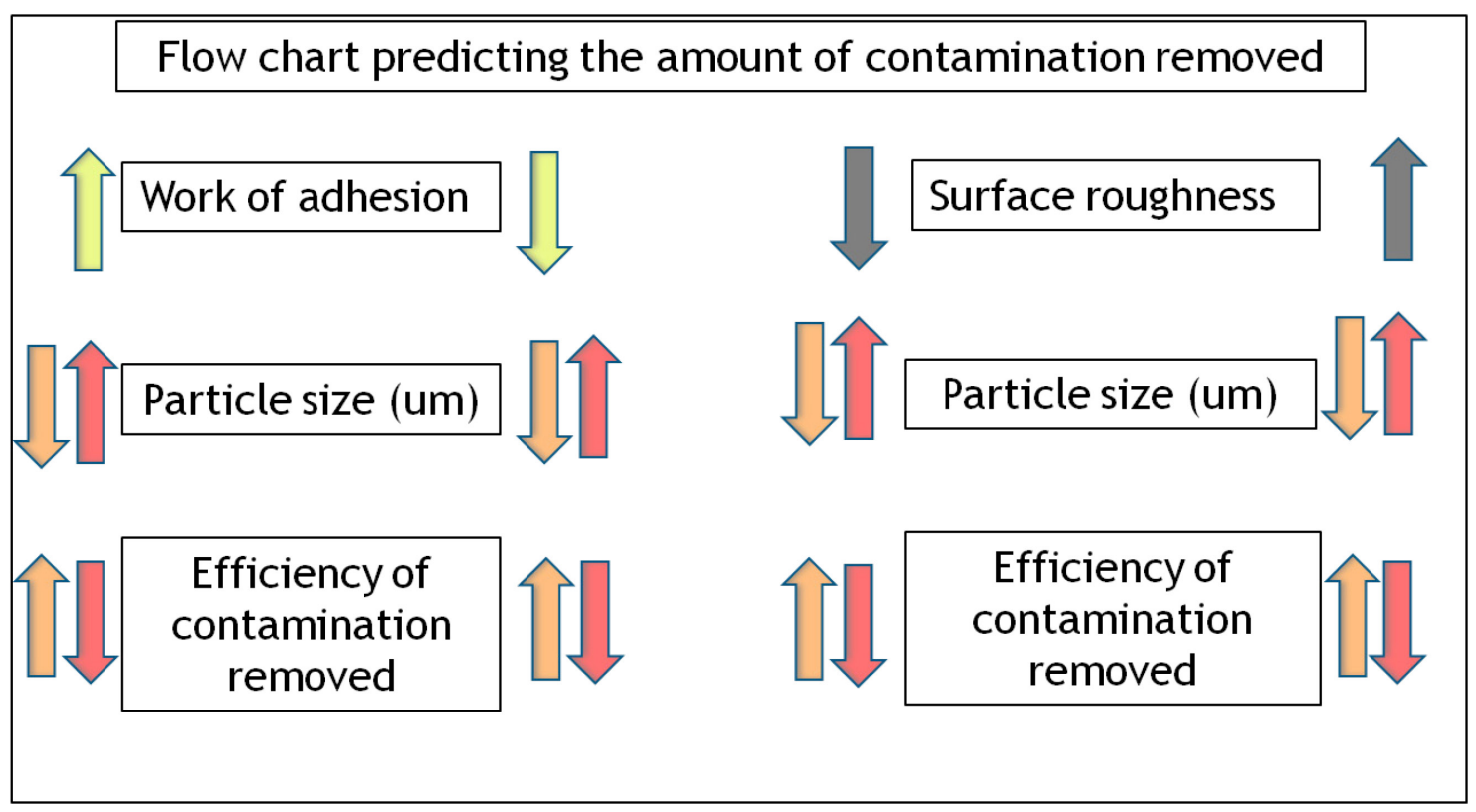

Figure 14 Flow chart predicting the amount of contamination removed The empirical model presented in Figure 14 predicts the amount of contamination removed, taking into consideration the terms of equation 4 that are directly proportional to the detachment force (particle radii and work of adhesion) and the roughness of the surface in which the contamination is present. 


\subsection{STATEMENT OF PURPOSE}

Dealing with scenarios in which loose contaminants are presented is a very complicated process because exposure to loose contaminants can cause internal deposition of hazardous chemicals (or unstable isotopes) to workers exposed directly or indirectly to the contaminated areas. Therefore, predicting the amount of contamination removed beforehand can help reduce this type of exposure. Mathematical theories developed so far explain the detachment force necessary to remove a particle from a surface but those theories neither account for the amount of contamination removed when cleaning a surface by wiping procedures nor account for the influence of surface roughness in the process of decontamination of surfaces exposed to environmental conditions. As a result, it is necessary to develop an empirical model able to predict the amount of contamination removed in terms of efficiency of loose contamination removal based on the variation of the factors explained by the JKR theory that determines the strength of the detachment force necessary to remove a particle from a surface, and the roughness of the surface.

\subsection{Research objectives}

The objective of this research is to evaluate the influence of the factors identified by the JKR theory (surface energy and particle size of the contaminant) that affect the strength of the detachment force necessary to remove a particle from a surface, and the roughness of the surface in which the contaminant is present, on the efficiency of removal of loose contamination. Achieving this goal will help to predict the values of removal for loose contamination based on similar scenarios and environmental conditions in which contamination is present. 


\subsection{Hypothesis}

It is hypothesized that: the interaction contaminant-surface (expressed by the surface energy, the roughness of the surface, and the particle size of the contaminant), and the interaction particle-wipe involved in the decontamination process, are predictors of the efficiency of loose contamination removal.

\subsection{Experimental hypothesis}

It is hypothesized that:

1. The effect of surface roughness contributes significantly to the efficiency of removal of loose contamination.

2. The effect of the particle size of the contaminant affects the efficiency of removal of loose contamination.

3. The type of wipe chosen in the process of cleaning influences the efficiency of removal of loose contamination.

4. The effect of the surface energy of the surface affects the efficiency of removal of loose contamination.

5. The interaction of the particle size, surface roughness, and wipe used significantly influences the efficiency of removal of loose contamination.

6. The interaction of the particle size, surface energy, and wipe used significantly influences the efficiency of removal of loose contamination. 


\subsection{MATERIALS AND METHODS}

The quantification of the contamination was determined by two methods. The first of the methods quantified the contamination removed by weighing the wipe before and after it was used to clean the surface; the contamination removed was the weight of the wipe after cleaning the surface minus the weight of the wipe before cleaning the surface. The second method involved the quantification of the contamination by radioactive detection of the contaminant on the surface and on the wipe. The quantification of the contamination removed by radioactive detection was achieved using two different types of disintegration modes: alpha and gamma. The alpha contamination was created using a powder of uranium oxide of particle size of $38 \mathrm{um}$. Uranium oxide is a natural alpha emitter which contains approximately $99.3 \%$ of U-238. The radioactive material in general has a low specific activity $(\sim 0.60 \mathrm{uCi} / \mathrm{g})$. The gamma contamination was created by technetium-99m which is a pure gamma emitter with gamma photons of an average energy of $140 \mathrm{KeV}$ and a half life of 6.02 hours. Technetium-99m was labeled to a Dowex 50Wx2 cationic resin in two particle sizes, $200(74-149 \mu \mathrm{m})$ and $400(37-74 \mu \mathrm{m})$ mesh.

In both radiation detection methods, the contamination was evaluated by the efficiency of loose contamination removal as defined the contamination before minus the contamination removed after cleaning the surface, divided by the contamination before cleaning the surface.

Before developing the methods to quantify the amount of contamination removed, the factors involved in the contaminant-surface and wipe-particle interaction were analyzed. 
This analysis involved an optical study of the wipes, an analysis of the surface roughness, and a determination of the surface energy for the surfaces used in the experiment.

\subsection{Method for characterization of the wipes}

The wipes were cut to squares, $7 \mathrm{~cm}$ by $7 \mathrm{~cm}$. All of the wipes used were composed of microfibers. The microfibers in wipes 1 and 3 are arranged in an organized pattern like a fish net, while the microfibers in wipe 2 (Chicopee-Masslin) and wipe 4 (Scott Multifold Towel manufactured by Kimberly-Clark) are randomly arranged. Also, wipe 2 has a surfactant attached to its microfibers. Those wipes were the top rag technology available in the market and are used by the Y-12 National Security Complex (in Oak Ridge, TN) to enhance the decontamination of surfaces by wiping procedures. The change in structure and composition of the wipes affect their surface energy, thus affecting the interaction between the contaminant and the wipe, and ultimately the amount of contamination removed from the surfaces.

The characterization method consisted of cutting each wipe into strips $(1 \mathrm{~cm}$ by $7 \mathrm{~cm})$ placing a drop of water on top of each wipe, and then, with the Drop Master equipment (used to measure contact angle, surface energy, and work of adhesion), evaluating the contact angle between the drop of water and the wipe. This study was carried out on wipes 1,2 , and 3 only, because wipe 4 instantly wet when the drop of water was placed on the surface of that wipe. The wipes were also studied under optical microscopy before and after being contaminated to see the distribution of the contaminant around the microfibers of the wipes. In addition, their behaviors in two media were studied. The first 
media was composed of a polar agent (water), and the second media was composed of a non-polar agent (a wood oil called Mounting Fluid Oil).

The procedure followed was to put three drops, $10 \mu 1$ each, of deionized water on each wipe sample and analyze under the microscope. Separately, another set of wipes were prepared and three drops, $10 \mu l$ each, of mineral oil was placed on top of each wipe. After that, another three drops of deionized water were placed on top of the wipes containing mineral oil. The observations were then conducted and the wipes analyzed. The microscope used was a MEIJI with a zoom of 10x in the lens and 10x ocular (Figure 15).

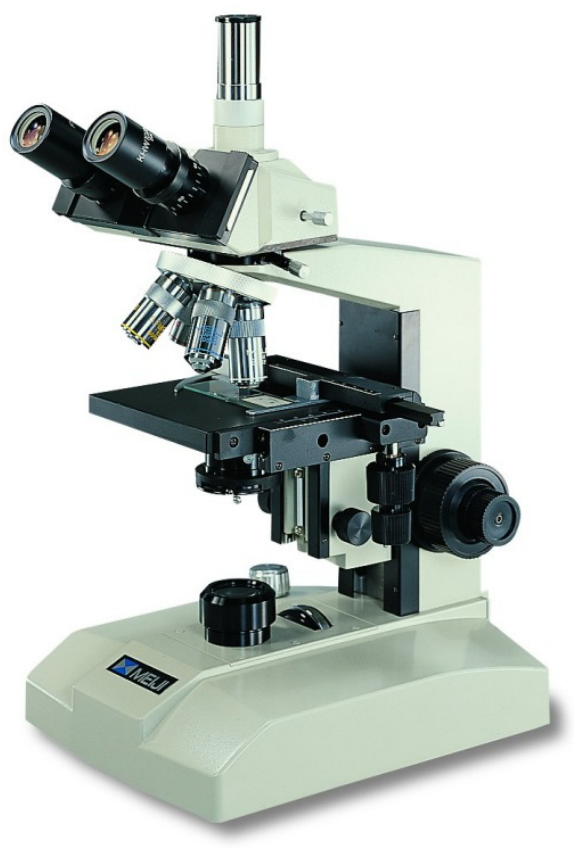

Figure 15 Wipes prepared to be studied under optical microscope Additionally, wipes 1, 2, and 3 were placed in a tube of $40 \mathrm{ml}$ with deionized water. The tubes were agitated manually and by a sonicator for $10 \mathrm{~min}$. After this, the wipes were separated from the water that was originally on the test tubes. Only the residual water that came from the Chicopee wipes changed in color (from transparent to yellow). Then it 
was suspected that the Chicopee wipes had a surfactant adhered to its microfibers. A Fisher Semiautomatic Model 21 Tensiomat (Figure 16) was used to measure the surface tension of the residual water. Ten measurements per water residual from each wipe were taken and a control was created with deionized water. This method helped to characterize the chemical adhered to the structure of the wipes. Also to study if this chemical present on the wipes affected the amount of contamination removed from the surfaces.

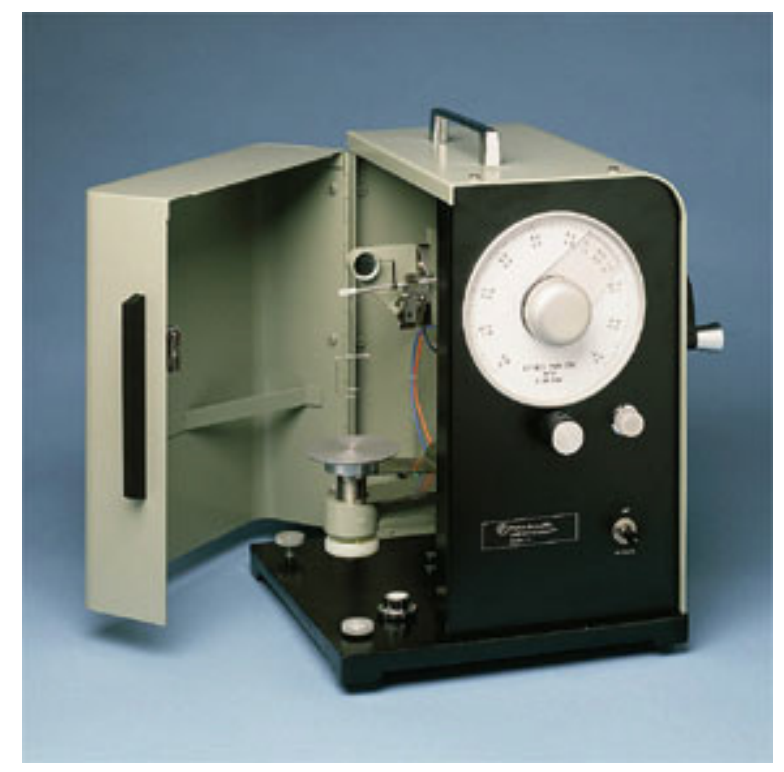

Figure 16 Fisher Semiautomatic Model 21 Tensiomat used to measured surface tension

\subsection{Preparation and selection of the surfaces}

The roughness of the surface is a factor that severely affects the removal of loose contamination from surfaces undergoing decontamination. Environmental surfaces are not perfectly smooth (Figure 12, section 2). Four surfaces were selected for this study: glass, PVC, tile and concrete (Figures 17 and 18, respectively). The surface roughness was measured using an Atomic Force Microscope (AFM). The microscope works on the principle of deflections of the cantilever and data acquisition when the cantilever gets 
closer to the surfaces. The method used to study the surface roughness under AFM was to select 2 pieces of each sample surface and perform a scan 5 times at randomly selected areas on each piece selected. An area of $2500 \mu \mathrm{m}^{2}$ was scanned on each of the sample analyzed. In the case of concrete, due to its high roughness, only 5 roughness analyses were made. The use of very rough surfaces in the AFM affected the cantilever to a point that could break it.

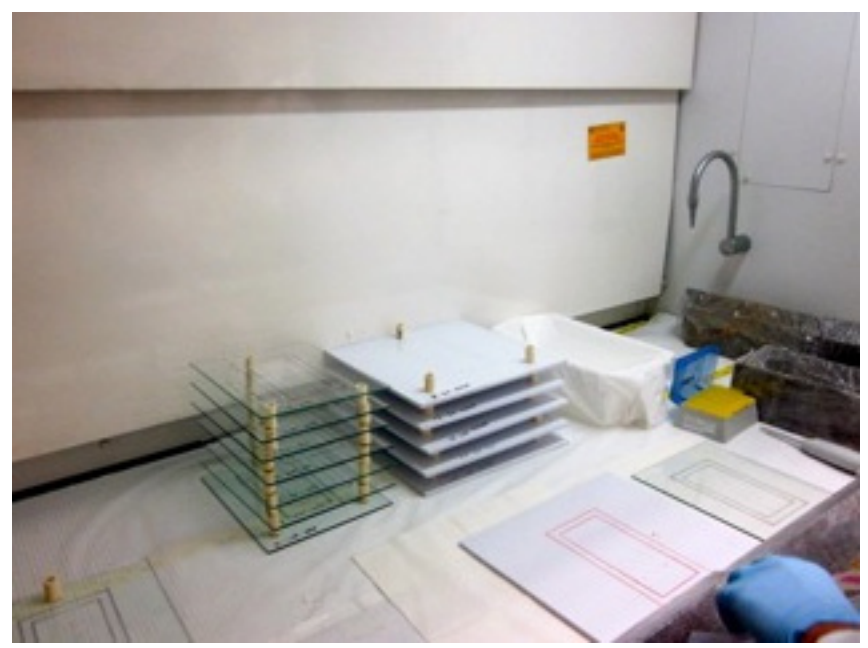

Figure 17 Surfaces of PVC and silica while being contaminated with the radioactive resin

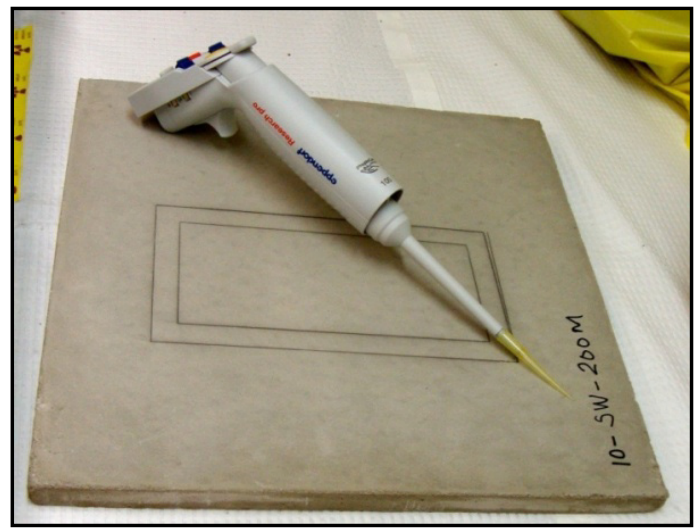

Figure 18 Surface of concrete ready to be used 
Additionally, the surface energy of the different surfaces was analyzed with the Drop Master equipment. The surfaces analyzed were PVC, Formica, tile, glass and concrete. Surface energy in solids (as explained in section 2.3.4) can be quantified by the contact angle formed between the sessile drop and the surface (Figure 12, section 2).

Samples were cut to a size able to fit into the Drop Master equipment from Kyowa Instruments (Figure 11, section 2). Ten pieces of PVC, Formica, tile, concrete (Figure 19), and glass were selected at random for each surface type and their wetting behavior were studied by measuring the angle formed by the drop and the surface at 10 locations selected by chance.

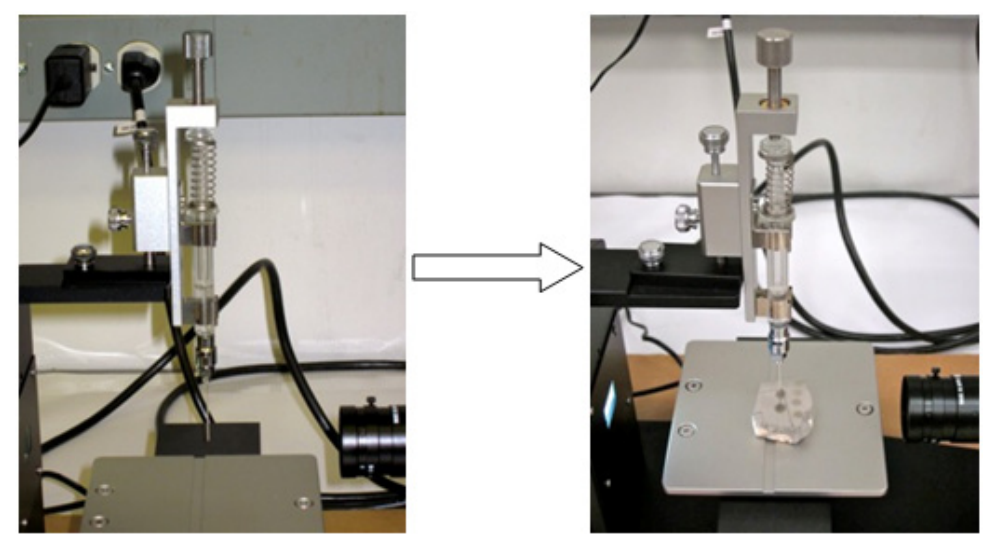

Figure 19 Drop Master Instrument, concrete surface under testing

\subsection{Method to quantify the contamination by weight}

This method was useful to test the hypothesis of the research by analyzing the interactions contaminant-wipe and contaminant-surface. The contaminant used was an insoluble thermoplastic pigment with an average size between $4-5 \mu \mathrm{m}$, and specific gravity of 1.36. The wetting behavior of the surfaces from PVC to tile (hydrophobic to 
hydrophilic) and the type of wipe used (a hydrophobic wipe, like wipe 1, and a absorbent wipe, like wipe 4) was varied.

This method was conducted into two phases: the first phase consisted of the preparation and spread of the contamination on the selected surfaces, and the second phase consisted of evaluating the contamination removed.

\subsubsection{Preparation and spread of the contaminant}

The contaminant used was a fluorescent powder in a particle size of $5 \mu \mathrm{m}$. The powder was a DayGlo A-594-5 blue fluorescing thermoplastic pigment (Figure 20). A weight of approximately $0.8 \mathrm{~g}$ of powder was mixed in $30 \mathrm{ml}$ of ethanol. Drops of $23 \mu 1$ were placed on each surface thirty times. The powder and ethanol mixture was manually agitated prior to each extraction. The surfaces were dried at room temperature for 24 hours.
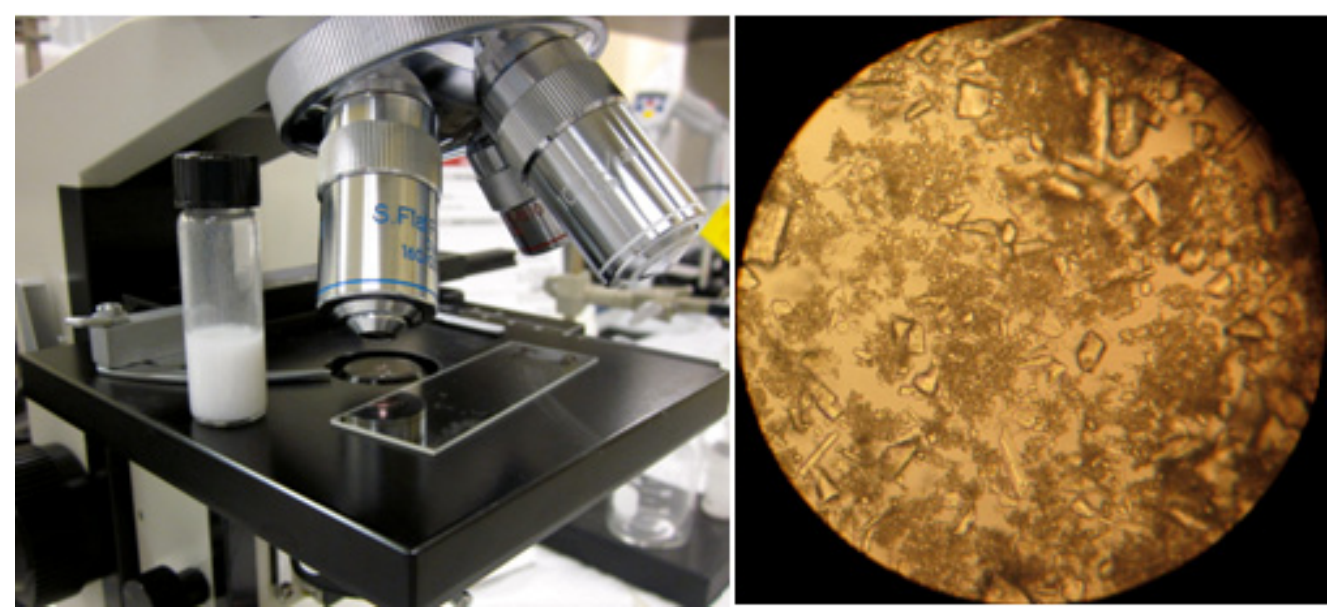

Figure 20 Fluorescent powder in ethanol solution (left), image in a 100x magnification of the same powder (right)

The wipes used for the evaluation were a microfiber wipe and a Scott Multifold Towel manufactured by Kimberly-Clark (control wipes). The wipes were cut to a size of $4 \mathrm{~cm}$ 
by $7 \mathrm{~cm}$ each. Ten test coupons each of Formica, tile and PVC were prepared for the experiment. The preparation consisted of labeling the area on each surface where the contamination was to be spread. Additionally, the test coupons were cleaned so that no dust remained on the surface prior to the process. They were then covered and stored prior to use.

\subsubsection{Experimental design}

For this experiment, the fluorescent powder was used to determinate the quantity of loose contamination removal. Statistical analysis followed a two by one factorial design (i.e. an Analysis of Variance (ANOVA)).

The factors and factor levels are shown in Table 1.

Table 1 Summary of Factors and Factors Levels

\begin{tabular}{|l|l|}
\hline Factor & Levels \\
\hline \multirow{2}{*}{ Wipes (A) } & Microfiber wipe (high level) \\
\cline { 2 - 2 } & Control Wipes (low level) \\
\hline
\end{tabular}

The experimental procedure for each technology (i.e., microfiber wipe and Scott Multifold Towels manufactured by Kimberly-Clark) was repeated in replications of ten for each of the three surfaces tested. A total of sixty surfaces were contaminated and decontaminated under this scenario. The results were then recorded in a table as depicted below for each surface and wipe used (Table 2).

Measurement of the contamination removed was determined by the difference between the weight of the wipe after wiping and the weight of the wipe before wiping the surface. The equipment used to measured the wipes before and after being contaminated was an analytical balance brand Toledo meter XS205. 
This was represented by:

$\rho=W P-W O P$

Where:

$\rho$ : is the amount of particles trapped by the wipe (in micrograms)

WP is the weight of the wipe after wiping the surface (in micrograms)

WOP is the weight of the wipe before wiping the surface (in micrograms)

Table 2 Data Collection per Surfaces

\begin{tabular}{|l|l|l|l|l|l|l|l|l|l|l|}
\hline \multicolumn{10}{|c|}{ Surface type } \\
\hline \multirow{2}{*}{ Wipes } & \multicolumn{1}{|c|}{ Replications } \\
\cline { 2 - 12 } & 1 & 2 & 3 & 4 & 5 & 6 & 7 & 8 & 9 & 10 \\
\hline Microfiber wipe & & & & & & & & & & \\
\hline Scott wipes & & & & & & & & & & \\
\hline
\end{tabular}

The hypothesis was given by:

$H_{o}: \mu_{1}=\mu_{2}$

$H_{1}: \mu_{1} \neq \mu_{2}$

where:

$\mu_{1}:$ is the mean value of the relative microfiber wipe (wipe 1) powder trapped

$\mu_{2}:$ is the mean value of the relative control wipe's (wipe 4) powder trapped

\subsection{Method for quantification of the contamination removed using ${ }^{238} \mathrm{U}$ presented in uranium oxide powder as contaminant}

This method helped to test the main hypothesis of the experiment in particles of $38 \mathrm{um}$ size. The contaminant used was uranium oxide $\left(\mathrm{U}_{3} \mathrm{O}_{8}\right)$. The alpha particles detected came 
from ${ }^{238} \mathrm{U}$ which constituted $99.3 \%$ by weight of the composition of uranium oxide. This form of uranium oxide is insoluble in water and the particles haves a size of $38 \mu \mathrm{m}$ in average. The wetting behavior of the surface was varied, as well as the type of wipe used. Wipes 1, 2, and 3 were used. The effect of the surface roughness on the amount of contamination collected on flat surfaces of concrete and non-polished stainless steel was also analyzed.

Prior to the deposition of the uranium oxide powder on the surfaces, the estimated number of counts per gram of uranium oxide needed to obtain a good signal to noise ratio was determined. Natural uranium has a specific activity of $0.7 \mu \mathrm{Ci} / \mathrm{g}$. The specific activity is defined as the amount of radioactive molecules divided by the number of total molecules present in a compound. Then, the specific activity of uranium oxide 0.71 (natural uranium specific activity) x 714 (molecular mass of uranium 238 present in the component) divided by 842.09 (the total molecular mass of the component) is $0.60 \mathrm{uCi} / \mathrm{g}$. This value represented the specific alpha activity within the uranium oxide powder.

Then, assuming that the minimum amount of contamination spread per surface was $1 \mathrm{mg}$, it can be obtained that:

$1 \mathrm{mg}=0.6 \times 10^{-3} \mathrm{uCi}$

$1 \mathrm{~Bq}=1$ disintegration per second

$1 \mathrm{Ci}=3.7 \times 10^{10} \mathrm{~Bq}$ from here $1 \mathrm{uCi}=3.7 \times 10^{4} \mathrm{~Bq}$

Then, the estimated counts per minute (cpm) in $1 \mathrm{mg}$ were: 
$C_{1 \mathrm{mg}}=\left(0.6 \times 10^{-3} \times 3.7 \times 10^{4} \mathrm{~Bq} \times 60\right) \mathrm{dpm} \times \eta($ detector efficiency $)=1332 \mathrm{dpm} \times$ $0.24=323 \mathrm{cpm}$

The detector efficiency was estimated by using a reference alpha source of ${ }^{239} \mathrm{Pu}$ with an estimated activity of $22732.8 \mathrm{dpm}$. After 15 measurements at different zones of the detector the mean numbers of counts were of 5514.72, which represented $24 \%$ of the real activity of the source used. This percentage is the detector efficiency.

The alpha background was between 1-10 cpm. Thus, with a small amount of powder, it was found to have a good signal to noise ratio. Additionally, as the U-238 has a long halflife, a counting time of 2 min per surface measurement was set for all the samples in order to decrease the uncertainty related to the decay mode. The amount of uranium oxide used never exceeded $10 \mathrm{mg}$.

\subsubsection{Procedure to contaminate the surfaces with uranium oxide.}

Due to the potential hazard of the contaminant used, this procedure was conducted under controlled conditions at the Radiological Laboratory OU-108 inside the glove box as shown in Figure 21.

ARC's Radiological Laboratory is equipped with state-of-the-art glove boxes, a filtration system, a fume hood, and a lead shielded enclosure for storage. The laboratory is licensed by the Florida Bureau of Radiation Control.

In this experiment, three different microfiber wipes were used. The wipes were cut to a 7 $\mathrm{cm}$ by $7 \mathrm{~cm}$ size and preserved in zip bags. 


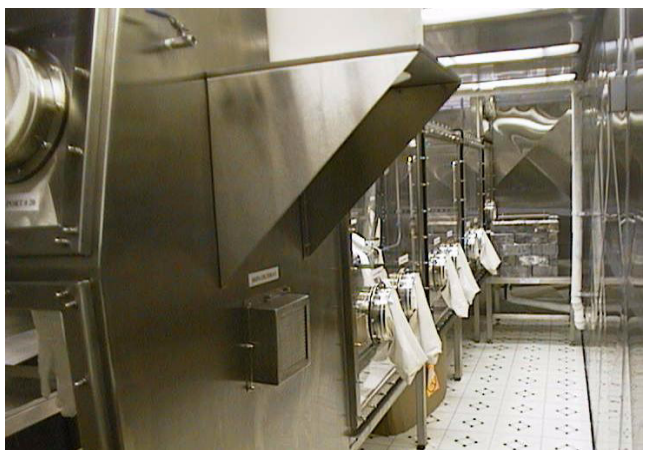

Figure 21 Glove box inside Radiological Laboratory OU-108

The surfaces selected for this evaluation included Formica, stainless steel, flat concrete, tile, and plastic. Each surface had dimensions of approximately $30 \mathrm{~cm}$ by $30 \mathrm{~cm}$.

The uranium oxide $\left(\mathrm{U}_{3} \mathrm{O}_{8}\right)$ was transported and opened inside the glove box to prevent any exposure during the experiments. Then, with a lab spoon, an activity in a range of 300 to 500 counts per two minutes was spiked on each surface in an area within the size of the detector. The alpha probe of the detector has an active area of $126 \mathrm{~cm}^{2}(16.6 \mathrm{~cm}$ length and $7.6 \mathrm{~cm}$ width). The alpha probe used was a scintillator $\mathrm{Zn}(\mathrm{Au})$ Ludlum model 43-90 with a Ludlum 2241-3 digital scaler.

Direct scans of the surfaces were performed to identify the amount of contamination present on the surface of the test coupons. When scanning for contamination, the detector was positioned to keep the same geometry of counts for all of the surfaces evaluated. The entire contaminated surface was measured using the digital survey meter (DSM) in the scalar mode for a 2 minute counting time. After that, the cleaning of the surface was performed following the trajectory shown in Figure 22 and the contaminated area was scanned again. 


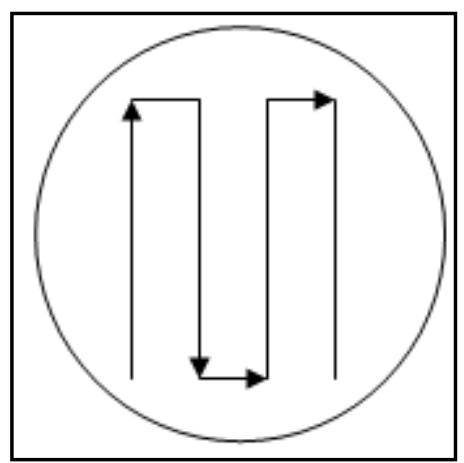

Figure 22 Wipe trajectory followed to decontaminated the surfaces

The counts were recorded before and after the wiping process. The ratio of these two counts was used to obtain the efficiency of loose contamination removal per surface and wipe used. Additionally, an air sampling pump (H-IQ CF model 901) was used for airborne measurement. The filter media air sampler was $4.7 \mathrm{~cm}$ in diameter and was supplied by Whatman International. The pumps were running before wiping the surfaces and ran until the wiping process was complete. Then, the contaminated filter paper was counted with the Ludlum probe to see if any airborne contamination was generated by the cleaning process.

A total of 75 surfaces were analyzed. An ANOVA method was used to compare the efficiency of contamination removal per surface contaminated and wipe used.

\subsection{Method to quantify the contamination removed using Dowex 50Wx2 labeled with $99 \mathrm{mTc}$ as contaminant}

The quantification of the contamination on the surfaces and on the wipes was obtained by the radioactive detection of technetium $99 \mathrm{~m}\left({ }^{99 \mathrm{~m}} \mathrm{Tc}\right)$ which is a pure gamma emitter with a half-life of 6.02 hours, and a gamma peak at $140 \mathrm{keV}$. The ${ }^{99 \mathrm{~m}} \mathrm{Tc}$ was attached to an inert cationic resin in two different particle sizes (200 and 400 mesh). The resin used was 
a Dowex 50Wx2 strong acid cation resin composed of a sulfonated polymer of styrene, ethylstyrene, $2 \%$ divinylbenzene (DVB) in hydrogen form and water. Once the resin was labeled with ${ }^{99 \mathrm{~m}} \mathrm{Tc}$, it was mixed with ethanol to accelerate the drying process, creating a homogeneous solution. After that, it was spread on the selected surface with a micropipette in drops of 10 microliters each, twenty drops in a selected area (Figure 23). The drops, after they were placed on the selected area, were allowed to dry for a day at room temperature.

Once the ethanol in the contaminant dried, the efficiency of loose contamination removed was obtained by measuring the number of counts before and after cleaning the surface. The efficiency of loose contamination removal was evaluated in two ways: first, by evaluating the ratio of the initial count rate on the surface minus the count rate on the surface after wiping and dividing by the initial count rate on the surface; and second, by calculating the ratio of the initial count rate on the surface minus the count rate present in the wipe after wiping, and dividing by the initial count rate on the surface. The experiment was divided into two phases. The first phase involved labeling the resin with the ${ }^{99 \mathrm{~m}} \mathrm{Tc}$ and testing the homogeneity of the contaminant between different particle sizes.

The second phase entailed the placement of the contamination on the surfaces in a known area, and finally decontamination of the surfaces.

\subsubsection{Method to label the Dowex $50 \mathrm{~W} \times 2$ resin with ${ }^{99 \mathrm{~m}} \mathrm{Tc}$ : radioactive resin generation}

The procedure followed to label the ${ }^{99 \mathrm{~m}} \mathrm{Tc}$ to the Dowex $50 \mathrm{~W} \times 2$ resin was divided into two phases. The first phase involved the chemical preparation of the stannous chloride II 
and the cleaning of the Dowex resin, while the second phase was for attaching the ${ }^{99 \mathrm{~m}} \mathrm{Tc}$ to the resin.

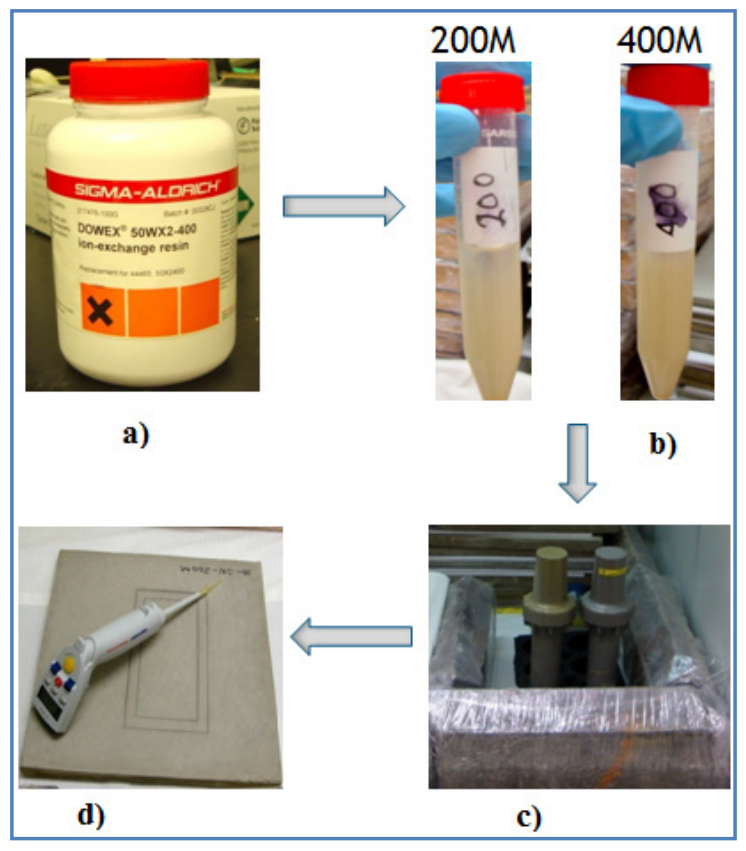

Figure 23 Experimental process of loose contamination generation. The resin was separated by particle size (74-149 $\mu \mathrm{m}$ and 37-74 $\mu \mathrm{m})$ and labeled with Tc-99m (items a. and b.). Then, it was shielded and spread in drops of $10 \mu 1$ on a selected area of a surface (items c. and d.)

\subsubsection{Chemical preparation and cleaning of the resin}

The chemical preparation was made by creating a $100 \mathrm{ml}$ solution of $0.1 \mathrm{M} \mathrm{HCl}$. Then, $200 \mathrm{mg}$ of stannous chloride was added to it. Separately, 1 gram of resin of size 200 and 400 mesh, respectively, were put in two centrifuge vials, along with a $5 \mathrm{ml}$ solution of $0.9 \% \mathrm{NaCl}$. Both tubes were centrifuged at $4500 \mathrm{rpm}$ and the supernatants were separated from the resins for each size. Finally, a second $5 \mathrm{ml}$ solution of $0.9 \% \mathrm{NaCl}$ was added to each vial. These procedures were conducted 24 hour before the labeling process started. At the moment the experiment started, the resins were centrifuged again and the supernatant extracted. 
4.5.1.2 Method followed to attach the ${ }^{99 \mathrm{~m}} \mathrm{Tc}$ to the cationic resin

Generally, the ${ }^{99 \mathrm{~m}} \mathrm{Tc}$ came in a pertechnetated $\left(\mathrm{TcO}_{4}{ }^{-}\right)$form in a $0.9 \%$ saline solution. The dose ordered each time never surpassed $5 \mathrm{mCi}$, and the volume in which the solution came ranged from $0.1 \mathrm{ml}$ to $5 \mathrm{ml}$. Once the dose arrived, the activity of ${ }^{99 \mathrm{~m}} \mathrm{Tc}$ and the time was recorded, then two sample volumes were extracted, equivalent to an activity of $1 \mathrm{mCi}$ each. Those volumes, together with $10 \mu \mathrm{l}$ of the stannous chloride mix (prepared in section 4.4.1.1), were added to each resin's vial prepared on the previous day. The volume of each vial was extended to $5 \mathrm{ml}$ with $0.9 \% \mathrm{NaCl}$. After that, the vials were centrifuged and the supernatant and resin in each vial were separated and measured. Finally, $10 \mathrm{ml}$ of ethanol (absolute, 200 proof) was added to each vial (Figure 24).

\subsubsection{Method to determine the appropriate ratio of concentration of $\mathrm{SnCl}_{2}$ to $\mathrm{TcO}_{4}{ }^{-}$ ions to yield a high tag of ${ }^{99 \mathrm{~m}}$ Tc to the cationic resin Dowex $50 \mathrm{~W}$ x 2}

The activity of $1 \mathrm{mCi}$ of ${ }^{99 \mathrm{~m}} \mathrm{Tc}$ ions presented in the solution was calculated by:

$$
A=\lambda \times N
$$

Where $\mathrm{A}$ is the activity of the sample in disintegration per seconds, $\lambda$ is the decay

constant of ${ }^{99 \mathrm{~m}} \mathrm{Tc}$, and $\mathrm{N}$ is the number of atoms of ${ }^{99 \mathrm{~m}} \mathrm{Tc}$ presented in a radioactive solution of technetium pertechnetate $\left(\mathrm{TcO}_{4}{ }^{-}\right)$. 


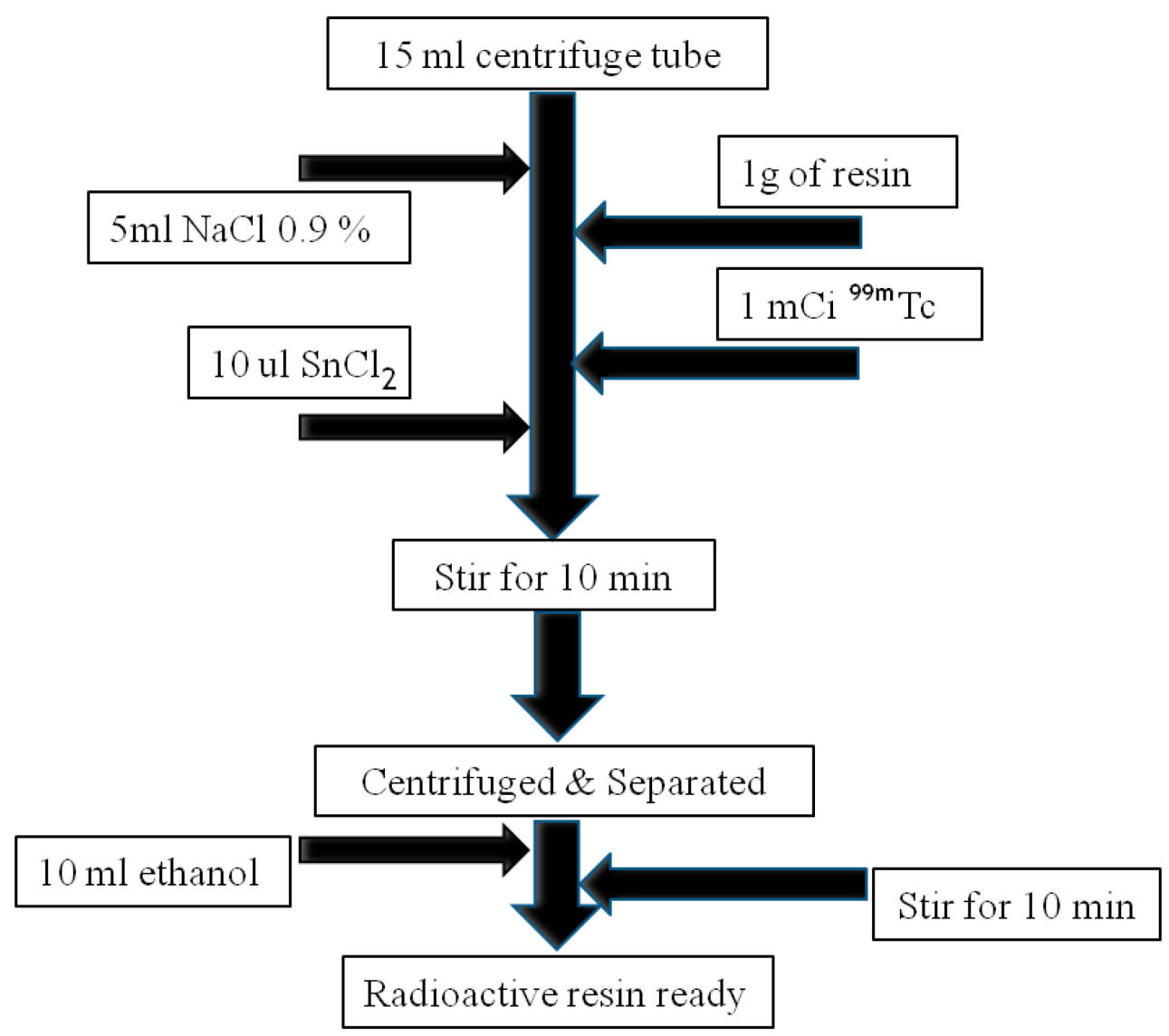

Figure 24 Summary of the resin labeling procedure

From equation 6 , the number of radioactive particles is:

$\mathrm{N}\left(\mathrm{TcO}_{4}^{-}\right)=\frac{\mathrm{A}}{\lambda}$

The number of moles was calculated by:

$\mathrm{n}\left(\mathrm{TcO}_{4}{ }^{-}\right)=\frac{\mathrm{N}\left(\mathrm{TcO}_{4}^{-}\right)}{\mathrm{N}_{\mathrm{A}}}$

Where $\mathrm{N}_{\mathrm{A}}$ is the number of Avogadro expressed in $\mathrm{mol}^{-1}$ (number of atoms in 1 gram mole). 
The concentration of technetium pertechnetate $\left(\mathrm{TcO}_{4}{ }^{-}\right)$is the number of moles divided by the volume of the solution of technetium pertechnetate $\left(\mathrm{TcO}_{4}{ }^{-}\right)$, then:

$\mathrm{C}\left(\mathrm{TcO}_{4}^{-}\right)=\frac{\mathrm{N}\left(\mathrm{TcO}_{4}^{-}\right)}{\mathrm{V}\left(\mathrm{TcO}_{4}^{-}\right)}$

Additionally, the number of moles of $\mathrm{SnCl}_{2}$ in the solution was found to be:

$\mathrm{n}\left(\mathrm{SnCl}_{2}\right)=\frac{\mathrm{m}\left(\mathrm{SnCl}_{2}\right)}{\mathrm{M}\left(\mathrm{SnCl}_{2}\right)}$

Finally, using the volume extracted from this solution after it was extended to $5 \mathrm{ml}$ with saline solution at $0.9 \% \mathrm{NaCl}$ (Figure 24), resulted in a final concentration of:

$\mathrm{C}\left(\mathrm{SnCl}_{2}\right)=\frac{\left[\mathrm{n}\left(\mathrm{SnCl}_{2}\right)\right] \mathrm{V}_{\mathrm{o}}}{\mathrm{V}_{\mathrm{f}}}$

Where $V_{o}$ and $V_{f}$ were the volume extracted from solution of equation 10 and the volume to which the solution was extended (in this case $5 \mathrm{ml}$ ), respectively.

Once the final concentration of stannous chloride II was obtained, the ratio of the concentration of stannous chloride II to pertechnatate ions was determined. The literature suggested that this ratio needed to be 1.5 to obtain a high number of reductions of the Tc ions [16].

\subsubsection{Particles preparation and characterization}

In order to enhance the ionic bond of the resin to the ${ }^{99 m} \mathrm{Tc}$, twenty-four hours prior to the experiment, one gram of resin was weighed and separated in two vials containing the particle size of 200 and 400 mesh, respectively. After that, $10 \mathrm{ml}$ of deionized water were added to both vials; soon the resins hydrated and also got rid of in-homogeneities present in the resins container (Figures 25 and 26). When the experiment began, both vials were 
centrifuged ( $3 \mathrm{~min}$ at $4200 \mathrm{rpm}$ ) and the suspended in-homogeneities (supernatant) were separated from the precipitated resin.

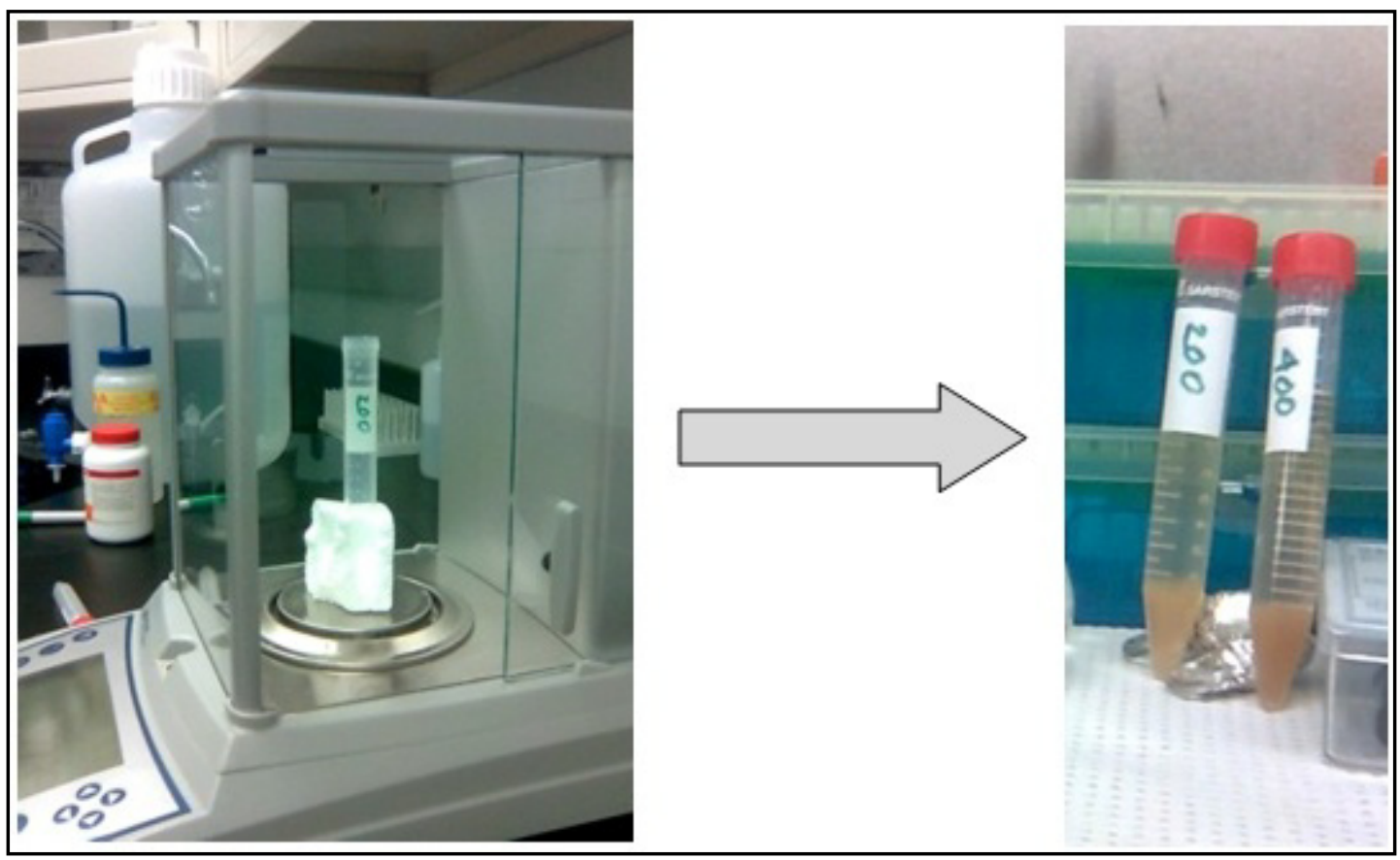

Figure 25 Separation by weight of approximately one gram of resin (left side) and two centrifugation vials with $10 \mathrm{ml}$ of deionized water with one gram of 200 and 400 mesh resins in each, respectively (right side)

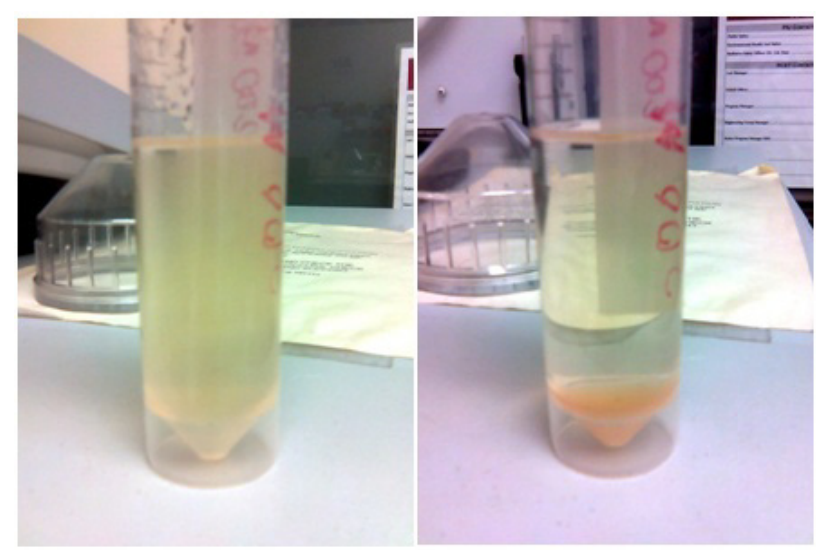

Figure 26 Resin of 200 mesh in deionized water after agitation (left side). Same resin after being centrifuged for $3 \mathrm{~min}$ at $4200 \mathrm{rpm}$ 


\subsubsection{Approach to evaluate the efficiency of labeling of ${ }^{99 m}$ Te to resins of 200 and 400 mesh, respectively}

The technetium $99 \mathrm{~m}\left({ }^{99 \mathrm{~m}} \mathrm{Tc}\right)$ isotope was received in a vial with an activity close to 5 $\mathrm{mCi}$ in a volume of approximately $5 \mathrm{ml}$. Two samples, with a volume equivalent to 1 $\mathrm{mCi}$ each, were extracted from the vial containing the isotope. After that, each sample was added to the vial containing the resin in a 200 and 400 mesh particle size (Figure 28). Then, a volume of $\mathrm{SnCl}_{2}$ capable of reducing the $\mathrm{Tc}^{99 \mathrm{~m}}$ ions to the values specified in section 4.5.2 was added. Both vials were stirred for 10 min at 25 degree Celsius; after that, they were centrifuged and the supernatant was separated from the resin each time for particles of 200 and 400 mesh, respectively. The activity of the supernatant and the resin were measured and these values were used to find the efficiency of the labeling process for the resins of 200 and 400 mesh by using equation 12 and 13, respectively.

$\eta_{200}=\frac{A_{i 200}-A_{s 200}}{A_{i 200}}$

$\eta_{400}=\frac{A_{i 400}-A_{s 400}}{A_{i 400}}$

where $A_{i 200}$ and $A_{i 400}$ were the initial activities of ${ }^{99 m}$ Tc in the solutions with particles of mesh sizes 200 and 400 respectively; and $A_{s 200}$ and $A_{s 400}$ were the activities of ${ }^{99 m}$ Tc in the supernatant of the solutions with particles of mesh sizes 200 and 400, respectively. Finally, $\eta_{200}$, and $\eta_{400}$ were the efficiency of the labeling of the ${ }^{99 \mathrm{~m}} \mathrm{Tc}$ to the resin in the particle size of 200 and 400 mesh, respectively. 


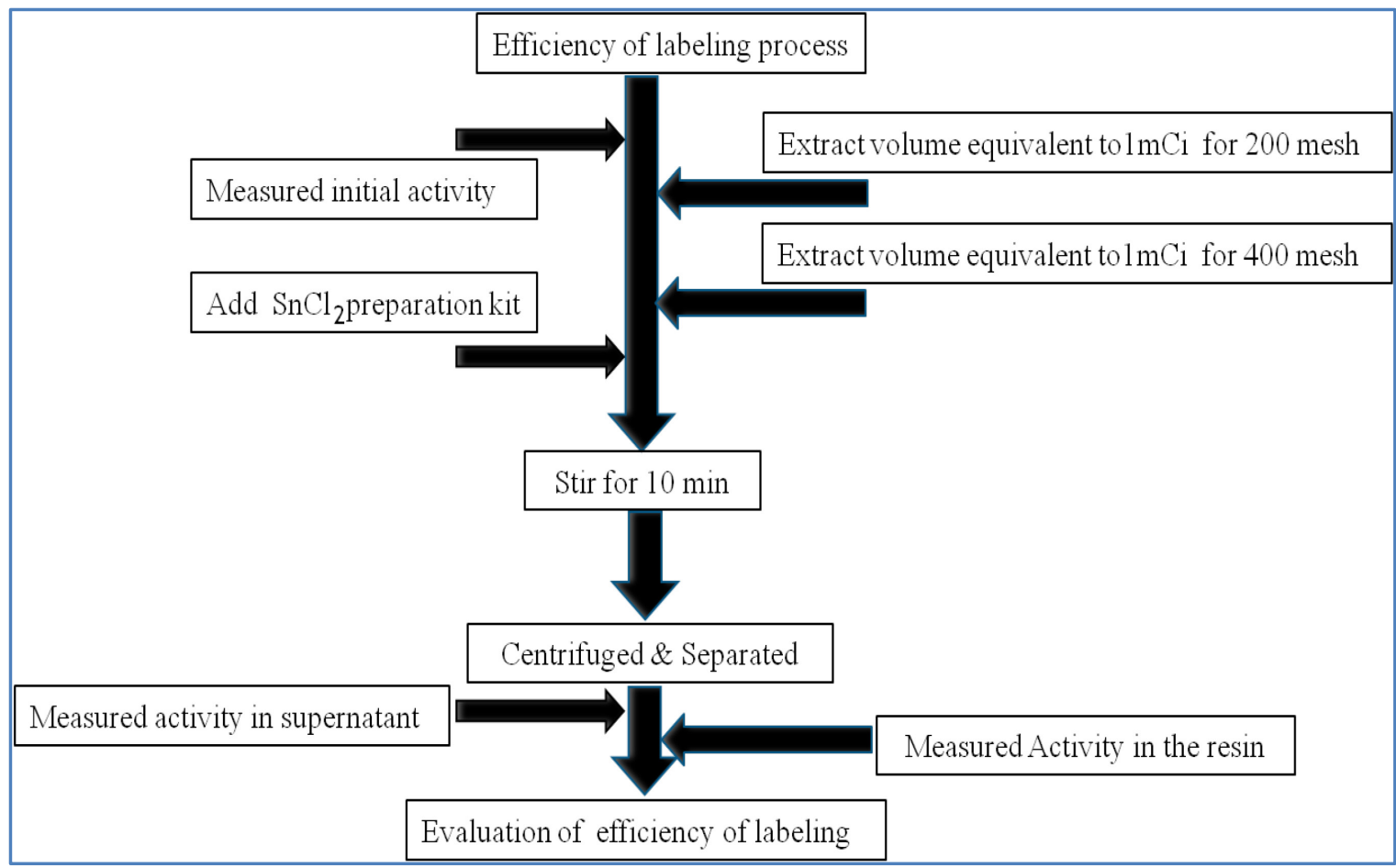

Figure 27 Flow chart followed to evaluate the efficiency of the labeling process of the Tc-99m to the Dowex 50Wx2 resin

\subsubsection{Method to evaluated the influence of ethanol in the ${ }^{99 \mathrm{~m}} \mathrm{Tc}$-resin bonding}

For the following experiment, the media for the labeling process was changed from deionized water to ethanol. It was conducted to see if this change would cause a rupture of the tagging between the ${ }^{99 \mathrm{~m}} \mathrm{Tc}$ and the cationic resin. Once the radioactive material was mixed in ethanol, it was centrifuged and the ethanol (supernatant) was separated from the resin (precipitated). Finally, the efficiency was calculated by:

$\eta=\frac{\mathrm{A}_{\mathrm{ir}}-\mathrm{A}_{\mathrm{se}}}{\mathrm{A}_{\mathrm{ir}}}$

Where $A_{\text {ir }}$ is the activity present in the resin with ethanol; and $A_{s e}$ is the activity of the supernatant (ethanol). Both activities were expressed in microcuries. 


\subsubsection{Method to determine the dispersion in number of counts of the contamination generated by the radioactive resin of 200 and 400 mesh}

As the labeling process was carried out for contaminants of different particle sizes, it was a concern if there were a significant difference between the mean value in count of the contaminant for $200(74-149 \mu \mathrm{m})$ and $400(37-74 \mu \mathrm{m})$ mesh. To evaluate this concern, a $10 \mu 1$ droplet of contaminant solution was added to each of forty test tubes (Figure 28). Twenty test tubes each contained $10 \mu 1$ of the contaminant solution with particles of mesh size 200 , while the remaining twenty contained $10 \mu 1$ of contaminant solution with particles of mesh size 400. A t-test was then performed to determine if there was a significant difference between mean values derived for each sample set. The t-test formulation was expressed as [9]:

$\mathrm{t}_{\mathrm{o}}=\frac{\overline{\mathrm{y}_{200}-\mathrm{y}_{400}}}{\mathrm{~s}_{\mathrm{p}} \sqrt{\frac{1}{\mathrm{n}_{200}}+\frac{1}{\mathrm{n}_{400}}}}$

And

$\mathrm{S}_{\mathrm{p}}=\frac{\left(\mathrm{n}_{200}-1\right) \mathrm{S}_{200}^{2}+\left(\mathrm{n}_{400^{-1}}\right) \mathrm{S}_{400}^{2}}{\mathrm{n}_{200}+\mathrm{n}_{400}-2}$

Where $\overline{y_{200}}$ and $\overline{y_{400}}$ are the mean value of counts recorded for the $10 \mu 1$ droplets of solution with particle mesh sizes of 200 and 400 , respectively; $n_{200}$ and $n_{400}$ are the number of test tubes with solutions of particle mesh sizes of 200 and 400, respectively.

Finally $\mathrm{S}_{200}$ and $\mathrm{S}_{400}$ are the standard deviations from the mean of the particle mesh sizes of 200 and 400, respectively. The null hypothesis was formulated as if the mean value of counts for the solutions with size 200 mesh was equal to the mean value of counts for the solutions with the size 400 mesh $\left(\mathrm{H}_{\mathrm{o}}: \overline{\mathrm{y}_{200}}=\overline{\mathrm{y}_{400}}\right)$. The alternative hypothesis was 
formulated as if the mean value of counts for the solutions with size $200(74-149 \mu \mathrm{m})$ mesh was different from the mean value of counts for the solutions with size 400 (37-74 $\mu \mathrm{m})$ mesh. $\left(\mathrm{H}_{1}: \overline{\mathrm{y}_{200}} \neq \overline{\mathrm{y}_{400}}\right)$.

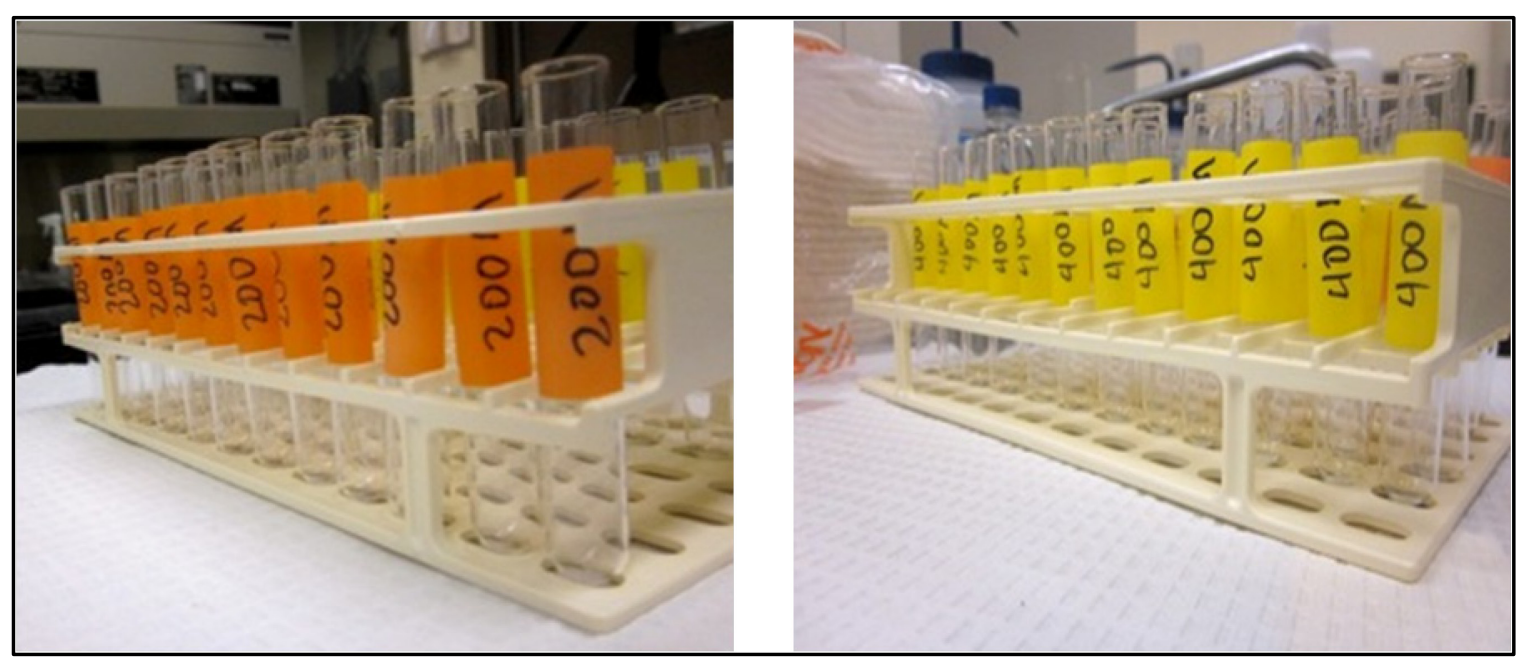

Figure 28 Test tubes used to check the variability in the mean number of counts of contamination of particles of $200(74-149 \mu \mathrm{m})$ and $400(37-74 \mu \mathrm{m}) \mathrm{mesh}$.

\subsubsection{Method to optimize the sample/background ratio based on number of counts}

As the contaminant was in a solution of ethanol, time for evaporation was necessary in order to allow the ethanol to evaporate and the contaminant to dry upon the surface. Then, the activity of the contaminant per surface (ion exchange resin labeled with ${ }^{99 \mathrm{~m}} \mathrm{Tc}$ ) was estimated prior to the experiment to allow a good sample to background ratio.

In the experiment, the concentration of resin in the solution was $0.066 \mathrm{~g} / \mathrm{ml}$ ( 1 gram in 15 milliliter), the volume of the drop was $0.01 \mathrm{ml}$, and the number of drops placed on the surface was 20 .

The total amount of the contaminant on the surface can be predicted by multiplying the concentration of resin times the volume of the drop times the number of drops per surface. Thus, the mass of resin per surface was approximately $13 \mathrm{mg}$. 
If it is assumed that the activity per unit mass of the resin is constant, then the activity placed on the surface can also be predicted by:

$A_{i} \times m_{f}=A_{f} \times m_{i}$

Where $A_{i}$ is the initial activity present in $m_{i}$ grams of resin and $A_{f}$ is the activity present in $\mathrm{m}_{\mathrm{f}}$ grams of resin. The activity per surface, $\mathrm{A}_{\mathrm{f}}$, corresponding to a mass, $\mathrm{m}_{\mathrm{f}}$, can be expressed in Becquerel (Bq). One $\mathrm{Bq}$ is equal to one disintegration per second. The number of disintegrations in $1 \mathrm{~min}$ (60 seconds) is:

$A_{f 60 \min }=A_{f} \times 60 \mathrm{~s}$,

Where $\mathrm{A}_{\mathrm{f} 60 \mathrm{~min}}$ is expressed in disintegrations per minute.

Then, the observed number of counts will be:

$\mathrm{A}_{\mathrm{o}}=\mathrm{A}_{\mathrm{f} 60 \mathrm{~min}} \mathrm{x} \eta_{\text {detector }}$

Where $\eta_{\text {detector }}$ is the detector efficiency.

After a drying period of 24 hours, the total counts per minute on each surface can be estimated by:

$$
\mathrm{A}=\mathrm{A}_{\mathrm{o}} \mathrm{e}^{-\lambda \mathrm{t}} \mathrm{cpm}
$$

Where $\mathrm{A}$ is the activity of the contamination (in counts per minutes) on the selected surfaces after 24 hour of drying that can be detected by the detector with efficiency $\eta_{\text {detector. }}$

In order to accurately estimate the efficiency of the detectors for Tc-99m, a pilot experiment was designed to obtain this value. The experiment was developed for the Eberline SHP 380 AB probe and the Packard Cobra 5003 detectors. 
4.5.7.1 Method to determine the efficiency of the SHP 380 AB probe and the Packard detector to Tc-99m

A dose of $1 \mathrm{mCi}$ was prepared per resin vial in $10 \mathrm{ml}$ of ethanol (see section 4.5.1), the activity per vial was measured in the dose calibrator Atom-Lab 100. The value of activity (mCi) equivalent to a volume of $10 \mu 1$ was reported. This value was multiplied by 20 which is the number of drops per surface used and expressed in disintegrations per minutes. Then, twenty two surfaces of silica and PVC (11 each) were spiked with the radioactive solution prepared in section 4.5.1 Twenty four hours later, the number of counts per minute on each surface every two minutes was recorded and at the end, a time correction was made respective to the initial reading recorded. Finally, the initial activity (measured by the dose calibrator) was decayed to the time the measurements on the surfaces were taken and the efficiency of the detector was calculated by:

$\eta_{S H P}=\frac{O}{R} * 100$

Where R,O accounted for the real number of counts per minute and the mean value of observed counts per minute, respectively

Separately, fifteen test tubes, each with a drop of $10 \mu$ of radioactive solution, were measured in the Packard detector twenty-four hours after the initial activity was measured. The value reported by the Packard detector on each test tube was averaged and multiplied by twenty. Finally, the efficiency of the detector was found by equation 21 . 


\subsubsection{Methods used to calculate the efficiency of loose contamination removed}

The efficiency of removal of loose contamination can be obtained experimentally by direct and indirect measurement methods. The first method calculates the contamination remaining on the surface by the following equation:

$\eta_{\text {direct }}=\frac{B-A_{S}}{B}$

where $\mathrm{B}$ is the amount of contamination before cleaning the surface, and $\mathrm{A}_{\mathrm{s}}$ is the amount of contamination remaining on the surface after it is decontaminated. The second method calculates the efficiency of removal contamination by calculating the amount of contamination on the wipe by:

$\eta_{\text {indirect }}=1-\left(\frac{B_{i}-A_{w}}{B_{i}}\right)$

where $B_{i}$ is the estimated initial amount of contamination and $A_{w}$ is the amount of contamination remaining on the wipe after cleaning the surface.

$\mathrm{B}_{\mathrm{i}}$ can be defined as:

$\mathrm{B}_{\mathrm{i}}=\mathrm{A}_{\mathrm{T}} \times \mathrm{N}_{\mathrm{D}}$

Where $A_{T}$ is the average number of counts detected in forty test tubes from section 4.1.4 and $\mathrm{N}_{\mathrm{D}}$ is the number of drops placed per surface.

If, from the homogeneity test presented in section in section 4.5.6, the mean number of counts per resin size is statistically different, then $\mathrm{B}$ is divided into two terms $\mathrm{B}_{\mathrm{i} 200}$ and $\mathrm{B}_{\mathrm{i} 400}$, representing the mean number of counts of particles of 200 and 400 mesh, 
respectively. Consequently, the efficiency of loose contamination measured by the indirect method is calculated by:

$\eta_{\text {indirect } 200}=1-\left(\frac{B_{i 200}-A_{w 200}}{B_{i 200}}\right)$

And

$\eta_{\text {indirect } 400}=1-\left(\frac{B_{i 400}-A_{w 400}}{B_{i 400}}\right)$

Where $A_{w 200}$, and $A_{w 400}$ is the amount of contamination remaining on the wipe after cleaning the surface with contaminants of 200 and 400 mesh, respectively.

\subsubsection{Experimental design \& Model generation}

To test the hypothesis, the experiment will follow a $2^{3}$ factorial design following an ANOVA method. Factorial designs are extensively used in experiments involving several factors where it is necessary to study the combined effect of the factors on a response [9]. Also, these types of designs allow making comparisons between significant interactions that might be discovered from the data obtained.

The factors and factor levels used to test the hypothesis are presented in Table 1. The null and alternative hypotheses of the experiment were tested by using a regression equation of the form:

$y=\beta_{o}+\beta_{1} x_{1}+\beta_{2} x_{2}+\beta_{3} x_{3}+\beta_{4} x_{1} x_{2}+\beta_{5} x_{1} x_{3}+\beta_{6} x_{2} x_{3}+\beta_{7} x_{1} x_{2} x_{3}$

where $y$ is the efficiency of loose contamination removal, $\beta_{\mathrm{o}}$ is the regression coefficient representing the mean value of the observations, $\beta_{1}, \beta_{2}, \beta_{3}$ are the regression coefficients of the effects of the particle size of the contaminant, roughness of the surface in which 
the contaminant is present, and the wipe used to decontaminated the surface, respectively. The rest of the regression coefficients $\left(\beta_{4}, \beta_{5}, \beta_{6}, \beta_{7}\right)$ reflect the contributions of all their possible interactions. Finally, $\mathrm{x}_{1}, \mathrm{x}_{2}, \mathrm{x}_{3}$ are the coded variables representing the particle size, the surface roughness and the wipe used, respectively.

A test for significance of regression was conducted to determine if there exists a linear relationship between the regression variables and the response. Then, the hypothesis can be set as:

$\mathrm{H}_{\mathrm{o}}: \beta_{1}=\beta_{2}=\ldots=\beta_{\mathrm{k}}=0 \quad 28$

$\mathrm{H}_{1}$ : at least one $\beta_{\mathrm{k}} \neq 0 \quad 29$

The null hypothesis $\mathrm{H}_{\mathrm{o}}$ states that none of the regression variables $x_{1}, x_{2} \ldots \ldots x_{k}$ contributes significantly to the model. The alternative hypothesis, $\mathrm{H}_{1}$, states otherwise.

The Analysis of Variance (ANOVA) test used to check the validity of $\mathrm{H}_{\mathrm{o}}$ was implemented by:

$$
S S_{T}=S S_{R}+S S_{E}
$$

Where: $\mathrm{SS}_{\mathrm{T}}$ is the total sum of squares, $\mathrm{SS}_{\mathrm{R}}$ is the regression sum of squares and $\mathrm{SS}_{\mathrm{E}}$ is the error sum of squares.

The table containing the experimental design is shown in Table 4. For the second row, starting in the first column, (1) represents the contamination removal value when using the factors A, B, and C (wipes, particle size and surface, respectively) at their low levels (which is the contamination removal value using the wipe 2, the particle size between 74 and 149 microns, and the rough surface such as concrete). The third row of the table 
represents the contamination removal value with factor A at high level (wipe 1) and factor B (particles between 74 and 149 microns) and C (concrete surface) at low levels, respectively, and so on, until the last row of the table in which the value of the contamination is recorded by using the highest value of the factors $\mathrm{A}, \mathrm{B}$, and $\mathrm{C}$ (wipe 1 , particles between 37-74 microns, and the plastic surface, respectively). The subsequent replications will be conducted in the same way and the order in which the samples will be recorded will be completely randomized. Once the roughness effect was characterized within the regression model, factor B was replaced by the surface energy effect

Table 3 Factor effect levels

\begin{tabular}{|l|l|}
\hline Factors & Factors Levels \\
\hline \multirow{2}{*}{ Wipes (A) } & Wipe 1 (high level) \\
\cline { 2 - 2 } & Wipe 2 (low level) \\
\hline \multirow{2}{*}{ Surfaces roughness(B) } & Smooth: PVC (high level) \\
\cline { 2 - 2 } & Rough: Concrete (low level) \\
\hline Particles size (C) & $37-74$ microns (high level) \\
\cline { 2 - 2 } & 74-149 microns (low level) \\
\hline
\end{tabular}

Table 4 Experimental design matrix

\begin{tabular}{|l|l|l|l|l|l|l|}
\hline Factor/ Combination & A & B & C & \multicolumn{3}{|l|}{ Efficiency of Contamination Removed } \\
\cline { 5 - 9 } & & & & Replication 1 & Replication 2 & Replication 3 \\
\hline$(1)$ & -1 & -1 & -1 & & & \\
\hline $\mathrm{a}$ & 1 & -1 & -1 & & & \\
\hline $\mathrm{b}$ & -1 & 1 & -1 & & & \\
\hline $\mathrm{c}$ & -1 & -1 & 1 & & & \\
\hline $\mathrm{ab}$ & 1 & 1 & -1 & & & \\
\hline $\mathrm{ac}$ & 1 & -1 & 1 & & & \\
\hline $\mathrm{bc}$ & -1 & 1 & 1 & & & \\
\hline $\mathrm{abc}$ & 1 & 1 & 1 & & & \\
\hline
\end{tabular}

This effect was evaluated in smooth glass (silica) and PVC surfaces. From the literature, glass surfaces had a marked hydrophilic behavior (high value of surface energy) while PVC surfaces had a hydrophobic (low value of surface energy) behavior. 


\subsubsection{Contamination \& decontamination procedures followed to populate the experimental design matrix}

The surfaces were contaminated with 20 (10 $\mu 1$ each) droplets of the solution prepared as described in section 4.5.1. The drops were placed in a confined area of the surfaces, within the size of the detector, then allowed to dry under the fume hood ventilation for 24 hours. The sample contamination as well as the decontamination was conducted in a random order. The cleaning of the surfaces was made matching the order imposed by the factorial method explained in section 4.5.9. The time at which each measurement was made, the type of wipe used, the surface number and the particle size of the contaminant was recorded. An overall distribution of the fume hood in which the process took place is presented in Figure 29. The green squares denote the spaces dedicated to placing the contaminated surfaces while the left side of the hood was used to decontaminate and take the readings.

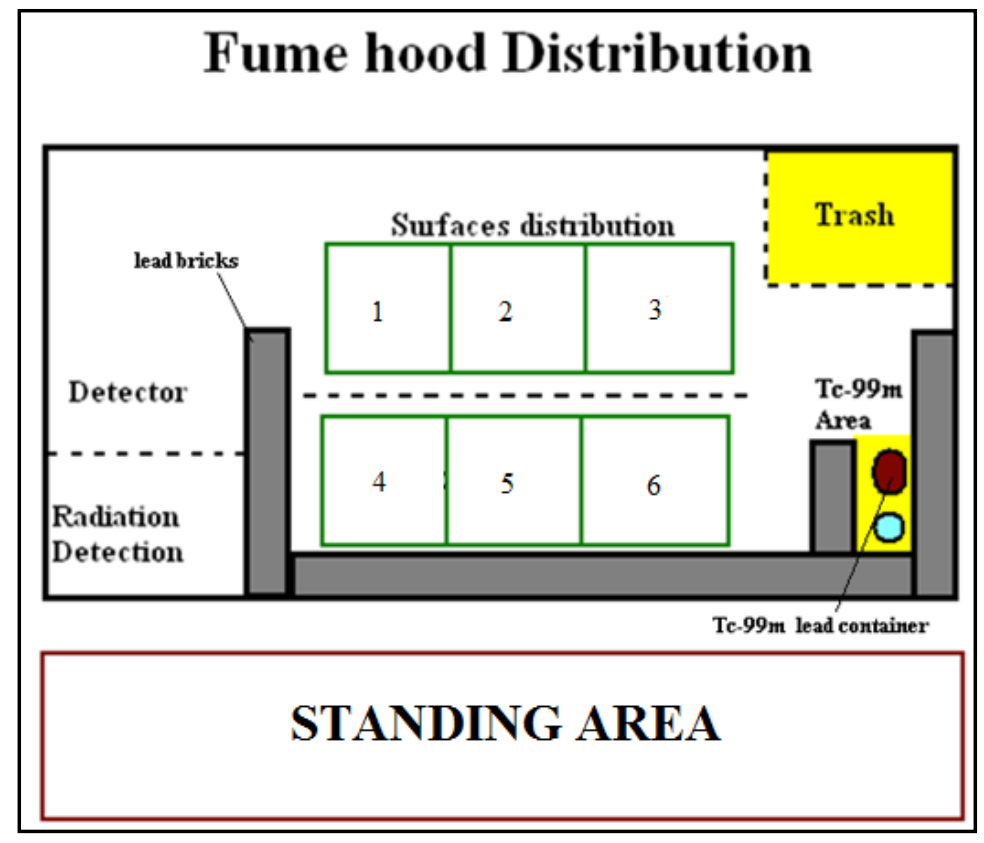

Figure 29 Fume hood work distribution area (top view) 


\subsubsection{Instrumentation used to quantify the contamination removed from the surfaces}

The detector used for detecting the contamination on the wipes was a Packard Cobra5003 multisampling well detector. It has a 3" x 3" NaI(Tl) crystal placed inside of a 2inch lead enclosure (Figure 30, right). The wipes were introduced into a test tube where the sample was analyzed. The detector for detecting the contamination on the surfaces was an Eberline SHP 380 AB probe with an Eberline E-600 instrument (Figure 30, left). It has a scintillation probe (SHP 380) made of $\mathrm{ZnS}(\mathrm{Ag})$. The active area is approximately $102 \mathrm{~cm}^{2}(68 \times 150 \mathrm{~mm})$.
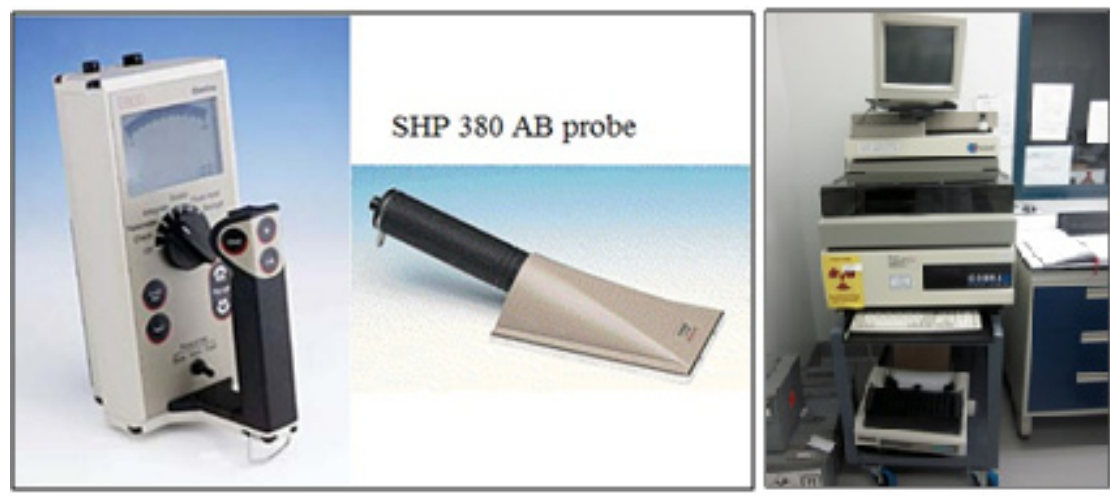

Figure 30 Eberline-E600 (left) and Cobra Packard II multisampling (right) detectors used on the experiments. 


\subsection{RESULTS}

The evaluation of the efficiency of loose contamination removal was achieved after setting a series of analytical and experimental conditions to fully characterize the process of contamination removal. Those conditions included physical characterization of the wipe and surface used. In addition, the influence of the factors predicted by the JKR theory was tested on the efficiency of loose contamination removal by wiping procedures on flat surfaces.

\subsection{Wipes and surfaces characterization.}

The wipes were studied under optical microscopy as explained in section 4 . When a drop of water was placed on wipe 1 and 3, the drop remained on the surface without any visual sign of interaction (Figure 31); however, when a drop of water was placed on wipe 2 and 4 (Chicopee-Masslin), they wetted completely. Additionally, when a drop of mounting oil was placed on another set of clean wipes, the microfibers of the wipes interacted with the mineral oil also (Figure 32).

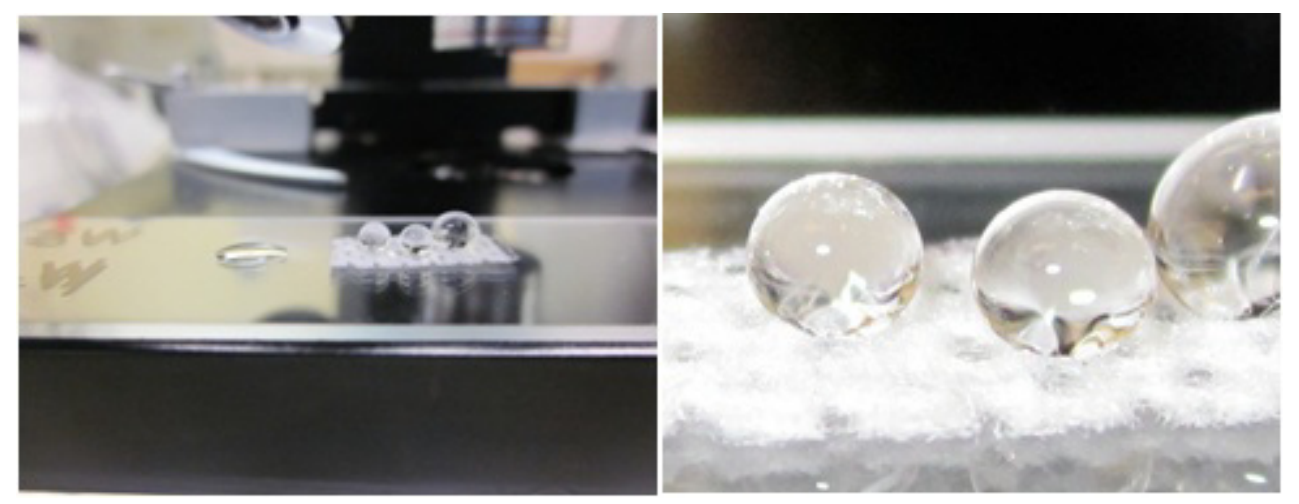

Figure 31 Wipe 1 with a 10 ul drop of deionized water. 
Wipe 2 interacted with a polar and a non polar media, absorbing the liquid both times. In order to explain this, each wipe was put in a $40 \mathrm{ml}$ glass, submerged in water and agitated with a sonicator for ten minutes (Figure 33). The water was then extracted from each vial (Figure 33, right) and the surface tension of the residual water was measured in the Fisher tensiomat 21 .

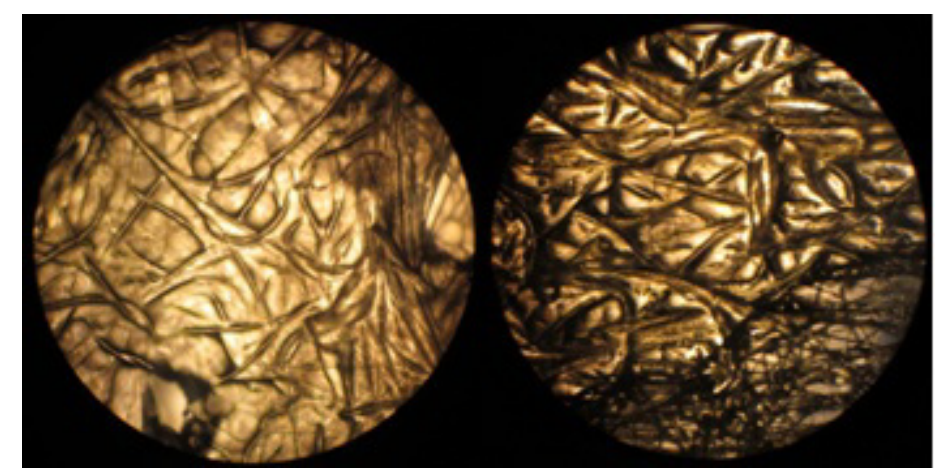

Figure 32 Wipe 1 and 2 (left and right, respectively) 100x magnification in presence of mounting oil

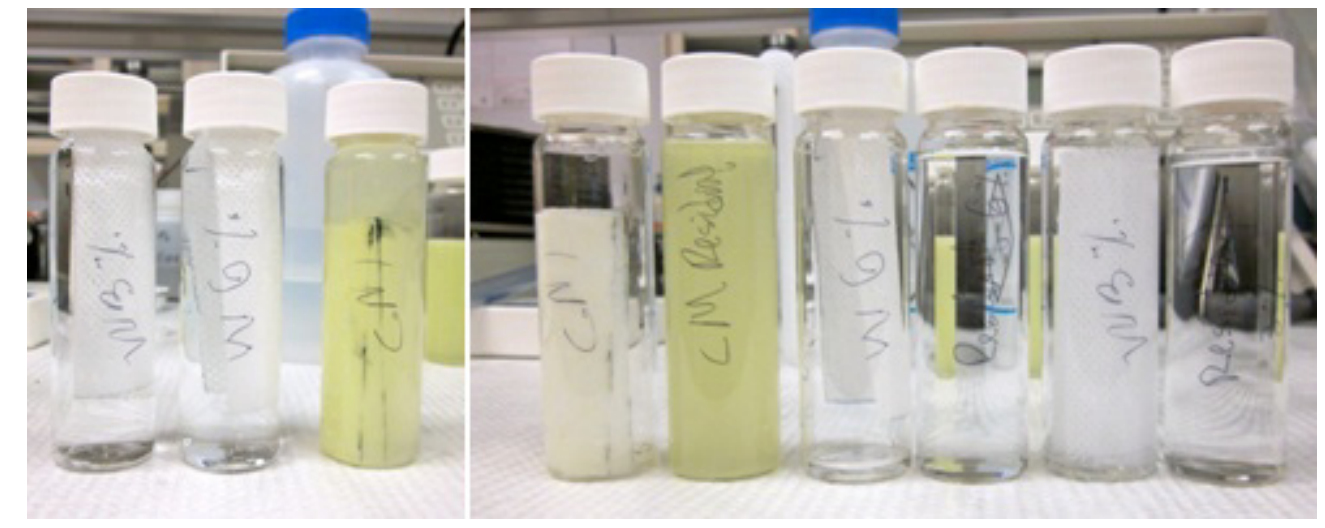

Figure 33 Wipes 3,1, and 2 submerged in water after being agitated with the sonicator for 10 min (left). After extracting the water from the wipes 2, 1 and 3 (right)

Table 5 summarized the results obtained with the tensiomat 21 equipment. The temperatures of the analyzed samples were each approximately $22^{\circ} \mathrm{C}$. From table 5 , the second column represents the apparent surface tension measurements made to a clean 
sample of deionized water (control), while the rest of the columns are the surface tension measurements from the residual water from each wipe. As it can be seen, the surface tension of the residual from wipe 2 has a value lower than that of water, while the surface tension of the residual from wipe 1 and 3 are similar to that of water.

Table 5 Summary of the surface tension values of the residuals from wipe exposure

\begin{tabular}{|c|c|c|c|c|}
\hline & \multicolumn{4}{|c|}{ Apparent Surface Tension (dyn/cm) } \\
\hline Sample & Deionized water (control) & Wipe 1 & Wipe 2 & Wipe 3 \\
\hline 1 & 71.9 & 73.8 & 45.9 & 71.9 \\
\hline 2 & 71.9 & 71.8 & 45.9 & 71.7 \\
\hline 3 & 72.0 & 71.8 & 45.9 & 72.4 \\
\hline 4 & 72.0 & 71.8 & 45.2 & 71.8 \\
\hline 5 & 72.0 & 71.8 & 45.2 & 71.9 \\
\hline 6 & 72.0 & 71.8 & 46.1 & 71.8 \\
\hline 7 & 72.0 & 71.9 & 45.2 & 71.7 \\
\hline 8 & 72.1 & 71.7 & 46.9 & 72.3 \\
\hline 9 & 72.0 & 71.7 & 46.2 & 71.7 \\
\hline 10 & 71.9 & 71.7 & 46 & 71.6 \\
\hline Average & $\mathbf{7 1 . 9}$ & $\mathbf{7 1 . 9}$ & $\mathbf{4 5 . 9}$ & $\mathbf{7 1 . 9}$ \\
\hline Stdev & $\mathbf{0 . 1}$ & $\mathbf{0 . 6}$ & $\mathbf{0 . 5}$ & $\mathbf{0 . 3}$ \\
\hline CV (\%) & $\mathbf{0 . 1}$ & $\mathbf{0 . 8}$ & $\mathbf{1 . 2}$ & $\mathbf{0 . 4}$ \\
\hline
\end{tabular}

This result, together with the optical evaluation, indicates the presence of a surfactant impregnated in wipe 2. Surfactants decrease the surface tension of water and in their composition have a polar and a non-polar head, which confirmed the absorption of the wipe in both polar and non polar media. The non-polar heads of the surfactant are attached to the hydrophobic microfiber of the wipe, while the polar heads of the surfactant remained unbounded, creating a polar cloth.

In order to confirm the statement of the paragraph above, the cleaned wipe 2 (presented in Figure 33, right side) was dried under vacuum for $30 \mathrm{~min}$ and later flushed with air for $10 \mathrm{~min}$. The resultant wipe, together with normal wipes 1,2 and 3 were taken under the 
Drop Master equipment to evaluate the contact angle. Table 6 summarizes the results obtained. Wipes 1 and 3 had a high contact angle which confirmed their hydrophobic behavior, while in the cleaned wipe 2 (Figure 34 and Table 6), drops started to appear but variations of the contact angle measured were high also (an indication of the surfactant remaining in the wipe). Wipe 2 was also measured as it came from the manufacturer; when the drops were placed on the surface of the wipe, they wetted completely, allowing no time to measure the contact angle (Figure 34).

Table 6 Contact angle measurement for wipe 1,2 (cleaned), and 3

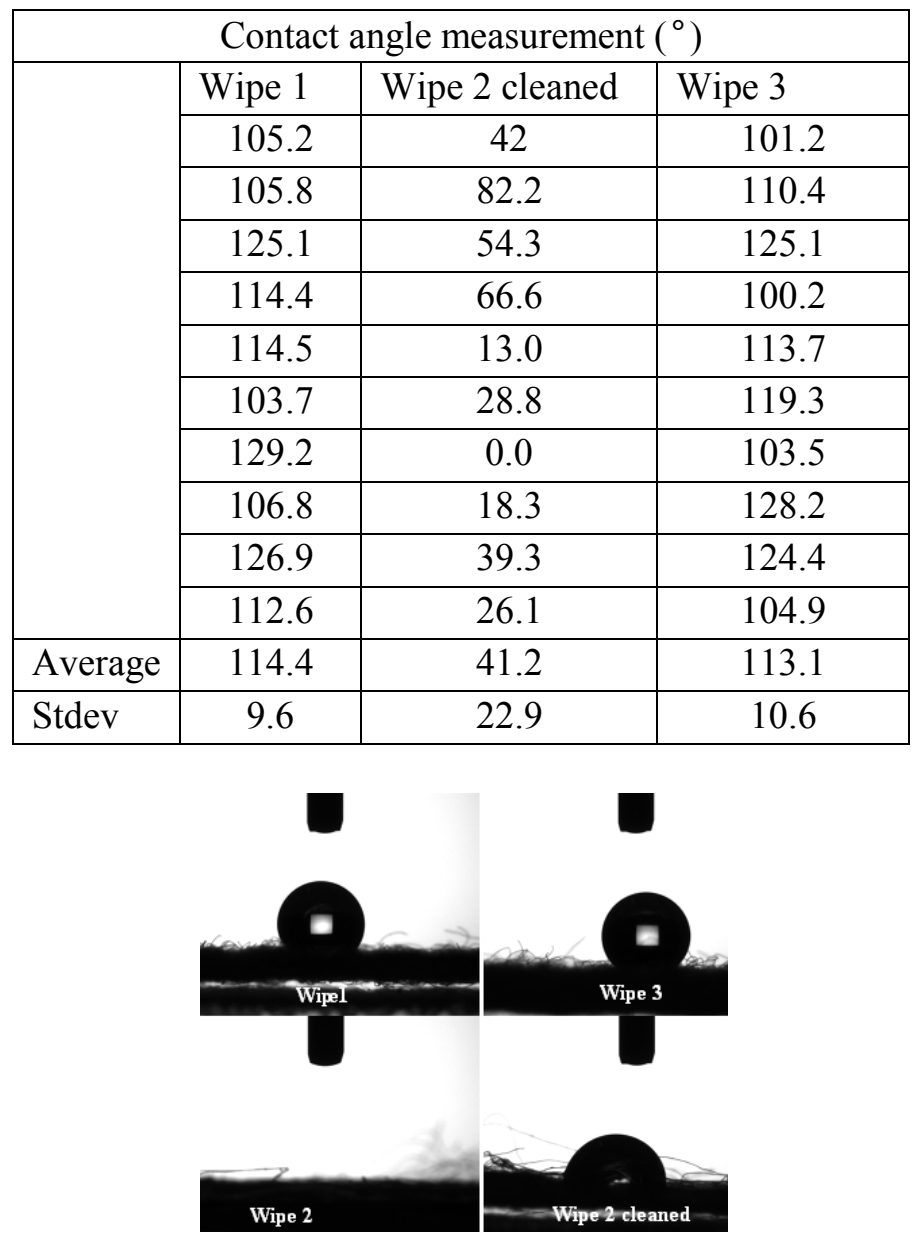

Figure 34 Wipes 1,2 and 3 study under the Drop Master equipment. Wipe 1 and 3 upper part of the image, wipe 2 lower part 


\subsubsection{Roughness analysis}

The roughness of the surface was characterized on four surfaces: PVC, silica, tile and concrete (Figure 35). The parameters presented in Table 7, $\mathrm{Rq}$ and $\mathrm{Ra}$, are indicators of the surface roughness [18]. In total, 25 scans were performed, 5 of concrete and 10 of silica, tile and $\mathrm{PVC}$, respectively. A high variability ( $\mathrm{CV}$ values in table 7) of the roughness of the surfaces evaluated was observed. This was expected because surfaces under environmental conditions are non-uniform, but it was also observed that the difference in roughness found between the concrete surfaces and the rest of the surfaces were approximately ten times higher (excepted for ceramic) which mean that concrete has a roughness significantly greater than the rest of the surfaces studied. However, for PVC and silica surfaces the difference in roughness was on the same order of magnitude, which indicated that in terms of roughness they were similar.

Table 7 Summary of roughness analysis made on the surfaces of concrete, PVC, tile and silica

\begin{tabular}{|l|l|l|l|l|l|l|l|l|}
\hline \multicolumn{4}{|l|}{ Concrete } & \multicolumn{2}{l|}{ PVC } & \multicolumn{2}{l|}{ Glass } & \multicolumn{2}{l|}{ Ceramic (Tile) } \\
\hline No. & Rq (um) & Ra (um) & Rq (um) & Ra (um) & Rq (um) & Ra (um) & Rq (um) & Ra(um \\
\hline 1 & 0.802 & 0.640 & 0.104 & 0.076 & 0.011 & 0.007 & 0.360 & 0.290 \\
\hline 2 & 0.708 & 0.517 & 0.092 & 0.064 & 0.009 & 0.007 & 0.240 & 0.180 \\
\hline 3 & 1.106 & 0.871 & 0.103 & 0.082 & 0.009 & 0.007 & 0.240 & 0.170 \\
\hline 4 & 0.968 & 0.788 & 0.104 & 0.074 & 0.01 & 0.008 & 0.210 & 0.150 \\
\hline 5 & 1.237 & 0.996 & 0.082 & 0.063 & 0.017 & 0.007 & 0.270 & 0.210 \\
\hline 6 & & & 0.128 & 0.097 & 0.013 & 0.008 & 0.250 & 0.210 \\
\hline 7 & & & 0.073 & 0.053 & 0.008 & 0.007 & 0.270 & 0.220 \\
\hline 8 & & & 0.088 & 0.062 & 0.008 & 0.007 & 0.170 & 0.120 \\
\hline 9 & & & 0.086 & 0.066 & 0.008 & 0.007 & 0.130 & 0.110 \\
\hline 10 & & & 0.116 & 0.094 & 0.009 & 0.007 & 0.290 & 0.230 \\
\hline Avera & $\mathbf{0 . 9 6 0}$ & $\mathbf{0 . 7 6 0}$ & $\mathbf{0 . 0 9 7}$ & $\mathbf{0 . 0 7 3}$ & $\mathbf{0 . 0 1 0}$ & $\mathbf{0 . 0 0 7}$ & $\mathbf{0 . 2 4 3}$ & $\mathbf{0 . 1 8 9}$ \\
\hline STDE & $\mathbf{0 . 2 1 0}$ & $\mathbf{0 . 1 8 0}$ & $\mathbf{0 . 0 1 6}$ & $\mathbf{0 . 0 1 4}$ & $\mathbf{0 . 0 0 3}$ & $\mathbf{0 . 0 0 0 4}$ & $\mathbf{0 . 0 6 3}$ & $\mathbf{0 . 0 5 4}$ \\
\hline CV & $\mathbf{0 . 2 2 4}$ & $\mathbf{0 . 2 4 7}$ & $\mathbf{0 . 1 7 0}$ & $\mathbf{0 . 1 9 6}$ & $\mathbf{0 . 2 8 0}$ & $\mathbf{0 . 5 8 5}$ & $\mathbf{0 . 2 6 2}$ & $\mathbf{0 . 2 8 8}$ \\
\hline
\end{tabular}




\subsubsection{Surface energy analysis}

Table 8 summarizes the results obtained from measuring the contact angle of a drop of water on surfaces of PVC, glass, tile, Formica and concrete (Figure 37).
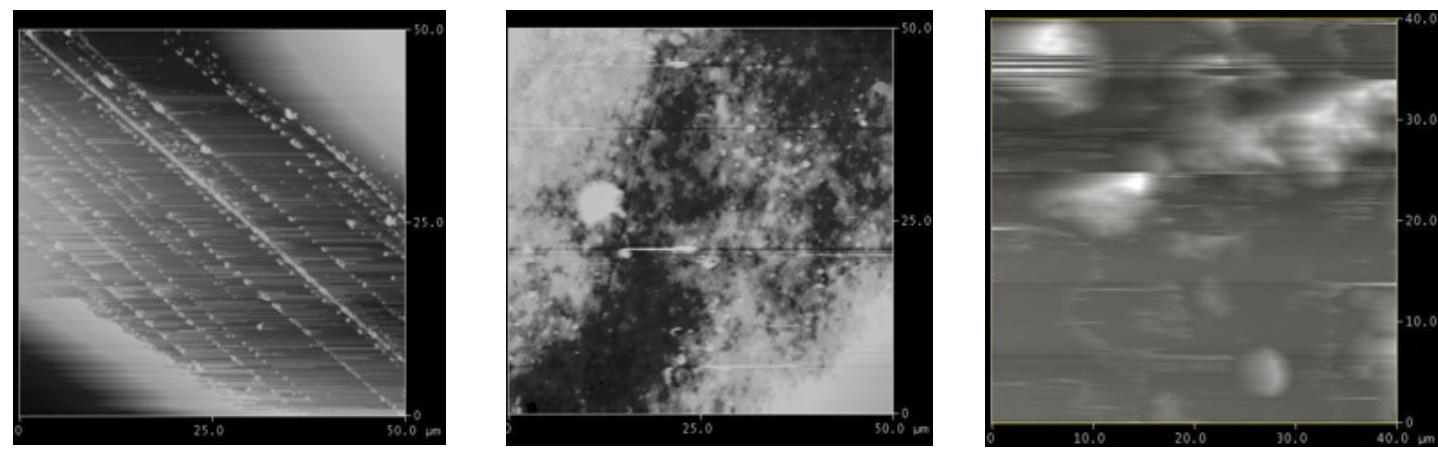

Figure 35 Atomic force microscopy of silica, PVC and concrete (left to right respectively)

Table 8 Contact angle for ten random selected surfaces of PVC, silica, and concrete, respectively

\begin{tabular}{|l|c|c|c|c|c|}
\hline \multirow{5}{*}{} & \multicolumn{5}{|c|}{ Contact angle $\left(^{\circ}\right)$} \\
\cline { 2 - 6 } & PVC & Glass & Concrete & Ceramic & Formica \\
\cline { 2 - 6 } & 95.1 & 50.0 & 44.9 & 40.7 & 69.9 \\
\cline { 2 - 6 } & 95.9 & 56.2 & 36.8 & 26.0 & 51.3 \\
\cline { 2 - 6 } & 92.1 & 55.3 & 36.4 & 24.9 & 53.7 \\
\cline { 2 - 6 } & 97.2 & 49.5 & 40.3 & 25.4 & 57.1 \\
\cline { 2 - 6 } & 98.2 & 45.4 & 51.0 & 24.0 & 48.2 \\
\cline { 2 - 6 } & 97.8 & 45.5 & 45.8 & 25.8 & 56.0 \\
\cline { 2 - 6 } & 99.4 & 44.4 & 40.8 & 25.5 & 64.8 \\
\cline { 2 - 6 } & 86.8 & 43.4 & 34.9 & 29.8 & 59.5 \\
\cline { 2 - 6 } & 89.3 & 38.3 & 34.9 & 24.6 & 69.7 \\
\cline { 2 - 6 } & 91.0 & 33.5 & 61.2 & 27.4 & 70.7 \\
\hline Mean & 94.3 & 46.2 & 42.7 & 27.4 & 60.1 \\
\hline STDEV & 4.2 & 7.0 & 8.4 & 4.9 & 8.2 \\
\hline
\end{tabular}

A high angle of contact between the sessile drop and the surface means a low value of surface energy (this indicates a surface with a remarkable hydrophobic behavior as shown in Figures 36 and 37) while a low angle of contact means a high value of surface energy 
(this indicates a surface with a remarkable hydrophilic behavior as shown in Figures 37 and 38 silica and concrete).

The JKR theory predicts that the detachment force depends on the contaminant size and on the work of adhesion between the particle and the surface. The work of adhesion is higher for particle-surfaces with similar values of surface energy than when they differ greatly from each other (a hydrophobic particle in a hydrophilic surface). As a result, it should be expected that this "lack of adhesion" will cause a difference in the amount of contamination removed from the surfaces. This statement was demonstrated in later sections.

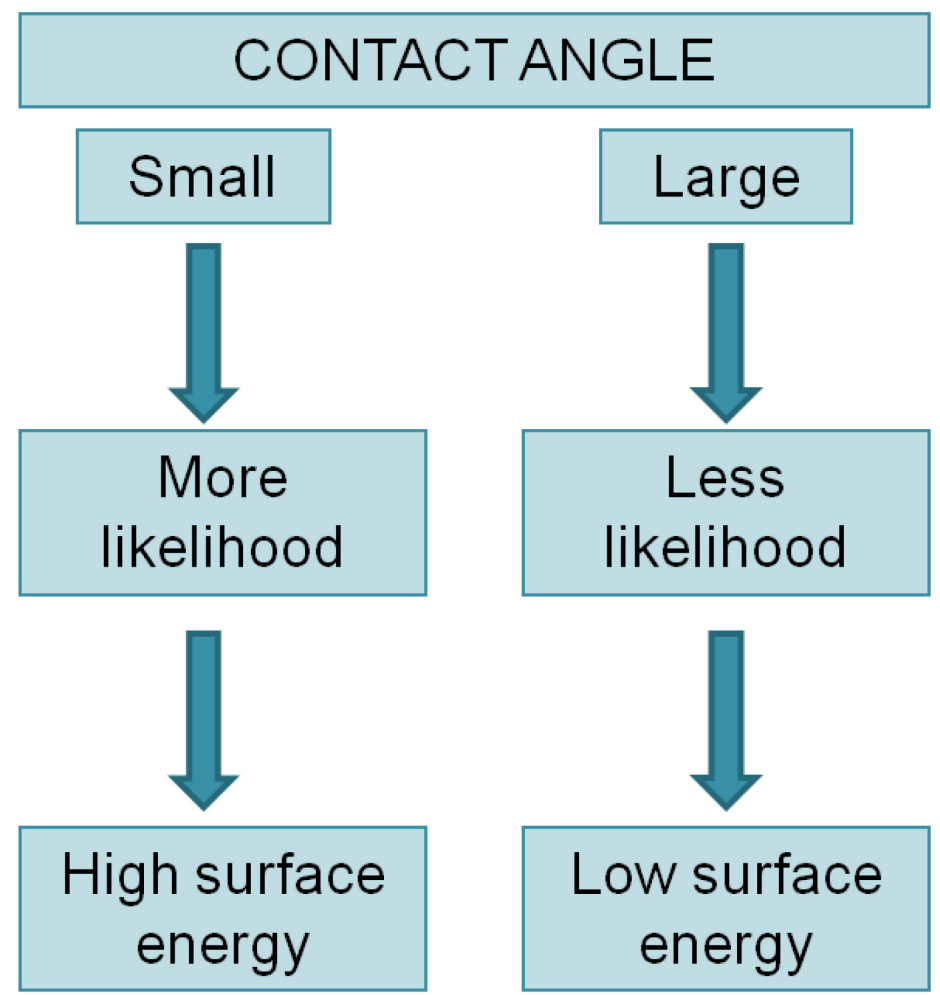

Figure 36 Contact angle \& surface wetting behavior flow chart 
The results presented in Table 8 are representative measurements of the contact angle of the surfaces analyzed. In non-homogeneous surfaces, like the ones studied, variations of the values presented above might occur, but overall, the composition of the surface will set the general tendency of the process of removal, unless there is a noticeable area with a different composition.
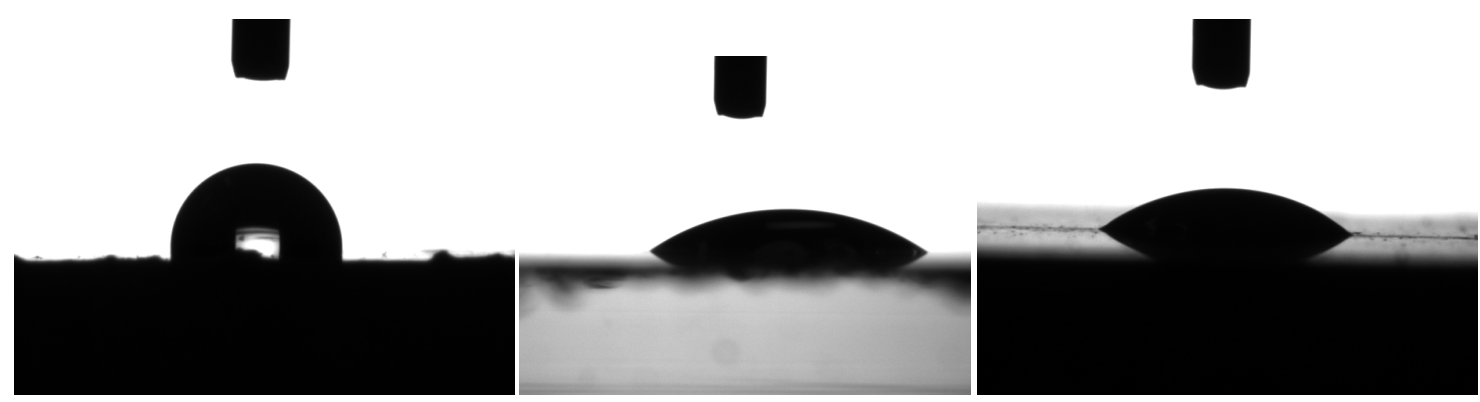

Figure 37 Surfaces of PVC, silica, and concrete (left to right in that order) under analysis into the Drop Master equipment

\subsection{Quantification of the contamination by weighing procedures}

The amount of contamination removed was quantified by weighing the wipe before and after cleaning the surface (Figure 38). The results are shown in Figure 39. It can be observed that wipe 1 removed more contamination per surface on each of the surfaces studied. Also, the Scott Multifold (wipe 4) wetted completely in the presence of water or oil, which means that their microfiber had an absorbent behavior, while wipe 1 is hydrophobic. The ANOVA table with a summary of the data collected is presented in Appendix 9.1.

The interaction between the contaminant and the wipe played an important role in the amount of contamination removed because when the contaminant and the wipe had similar surface energy behavior, the amount of powder removed decreased (Figure 40 
wipe 1 and Scott multifold value of powder removed); while, when their surface energy greatly mismatched, their interaction decreased and more contamination was removed from the surfaces. In addition, it was observed that when the tile surfaces were used, the amount of contamination removed increased in comparison to PVC; this was caused due to a change in surface energy of the surface (interaction contaminant-surface, ceramic surfaces are hydrophilic, see section 5.1). The work of adhesion for contaminant-surface of similar composition (thermoplastic pigment-PVC) is greater than when their composition differs (thermoplastic pigment-ceramic), thus affecting the detachment force, and in consequence the amount of contamination removed. This observation was verified for each wipe used (Figure 39).
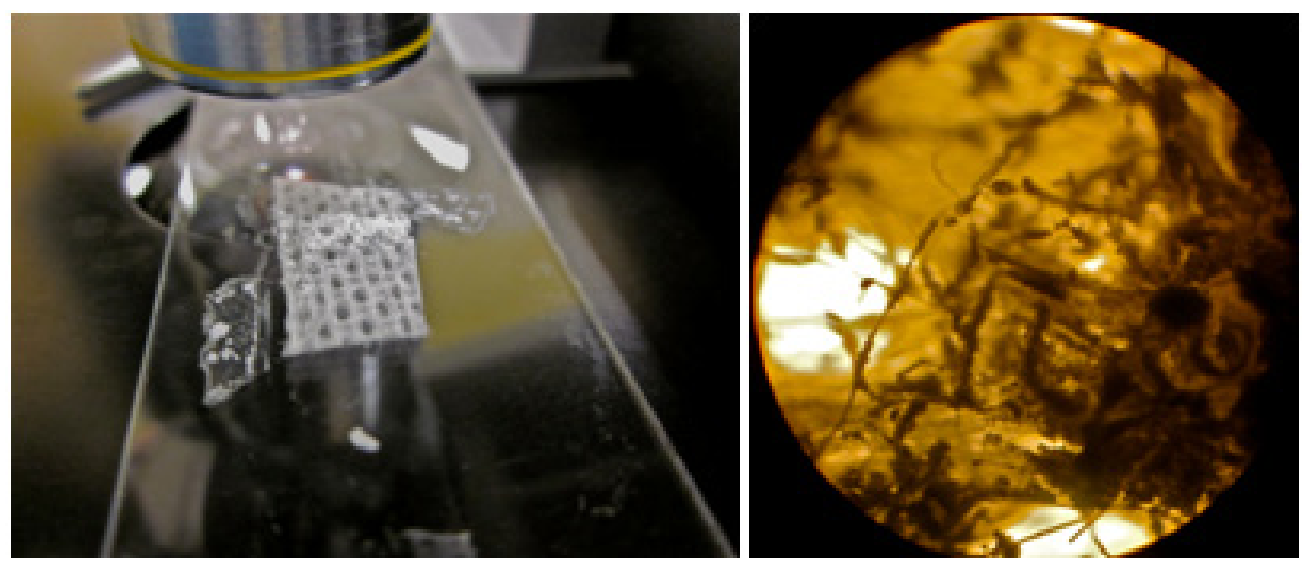

Figure 38 Wipe 1 under optical microscope after being contaminated with the fluorescent powder 


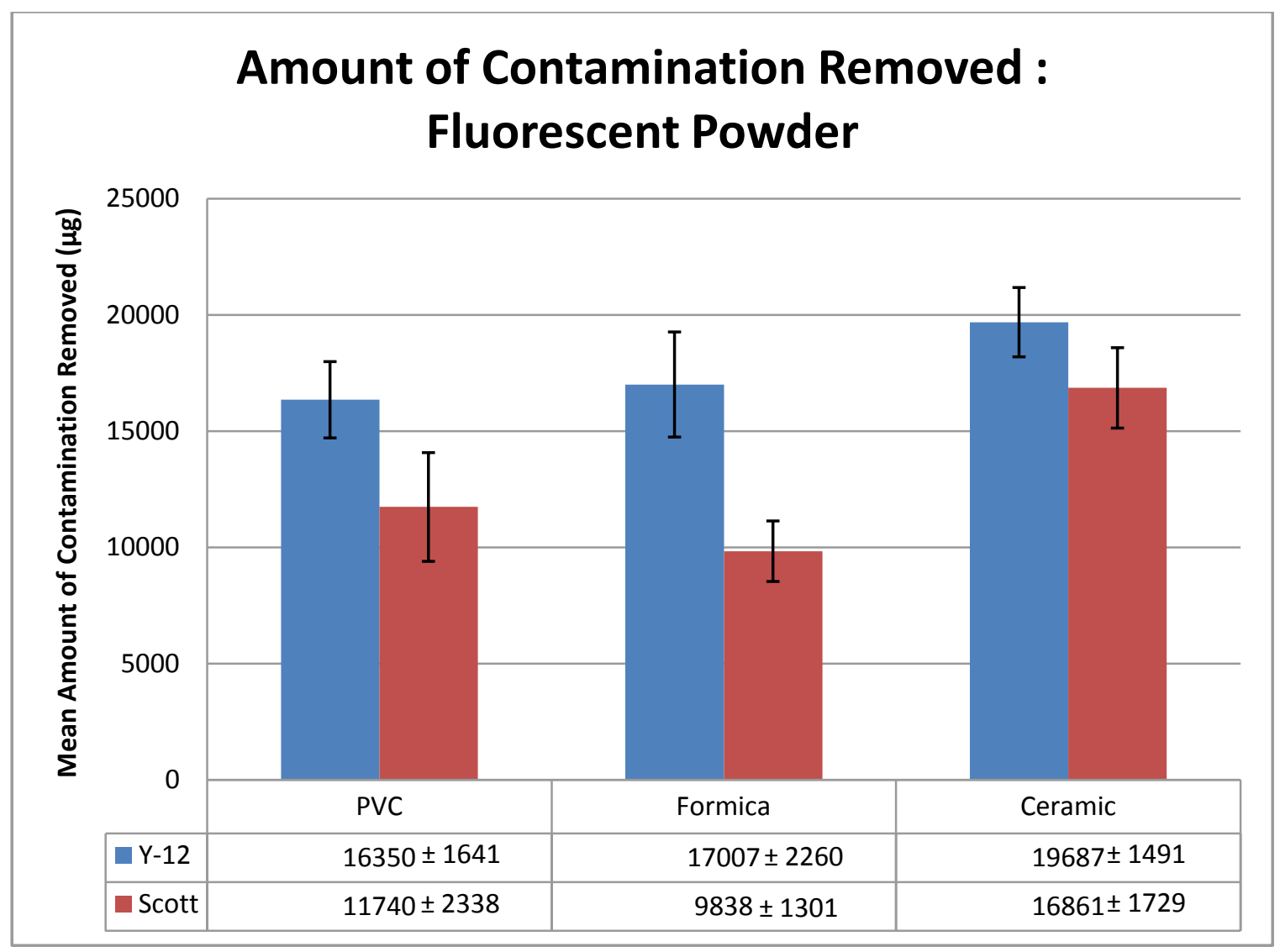

Figure 39 Summary of the weighing procedure to estimate the contamination of surfaces. The data is presented as the mean amount of contamination removed \pm the standard deviation, both expressed in micrograms (Tables 33 through 35, appendix 9.1)

Figure 39 presented a summary of the results analyzed. Ten replications per wipe were done on each surface analyzed. The values of the standard deviation of the observations are also presented. An ANOVA method was applied to the data to test the significance of the difference observed (appendix 9.1), concluding that the difference found was significant (small P-value, appendix 9.1). 


\subsection{Quantification of the contamination removed by radioactive detection, alpha disintegration mode}

Tables 36 through 40 (Appendix 9.2) shows the results based on wiping various surfaces using 3 wiping materials (wipe 3, wipe 1 and wipe 2, the baseline technology). Table 36 presents results for plastic (PVC), Table 37 for ceramic tile, Table 38 for concrete, Table 39 for Formica and Table 34for stainless steel. A $100 \mathrm{~cm}^{2}$ area $\mathrm{ZnS}(\mathrm{Ag})$ alpha counter of predetermined counting efficiency was used for counting all samples. Column 1 of the tables shows the experiment number and columns 2, 3 and 4 show the net alpha counts (after background subtraction) in 2 minutes on the stated surface contaminated with $\mathrm{U}_{3} \mathrm{O}_{8}$ before wiping with wipe 3, wipe 1 (Y-12 wipes) and Chicopee Masslinn, respectively. Columns 5, 6 and 7 show the percentages of activity removed from the surface using these wiping media and are obtained from the ratio of the difference in the initial and final radioactivity on the surface and the initial radioactivity. Columns 8, 9 and 10 give the percentage of net counts on the air sampling filter paper (run during wiping of the surface and about 5 minutes post wiping), and counts on the surface before wiping.

Tables 36 through 40 (appendix 9.2) also show mean, standard deviation and the random standard uncertainty (standard error) of decontamination (removal) efficiency of the wiping media for various types of surfaces, as well as radioactivity on the air sampling filter.

For smooth surfaces such as plastic, ceramic tiles and Formica, there is no significant difference in the decontamination efficiency of the three wiping media. For rough surfaces such as concrete and stainless steel (un-buffed, unpolished), the decontamination efficiency increases from wipe 3 to wipe 1 (Y-12 Wipes) to wipe 2 (Chicopee Masslinn). 
The moist nature of Chicopee Maslin may lend it to make better contact with the material in the pores and hence may result in higher removal efficiency compared to other wiping media, which are dry.

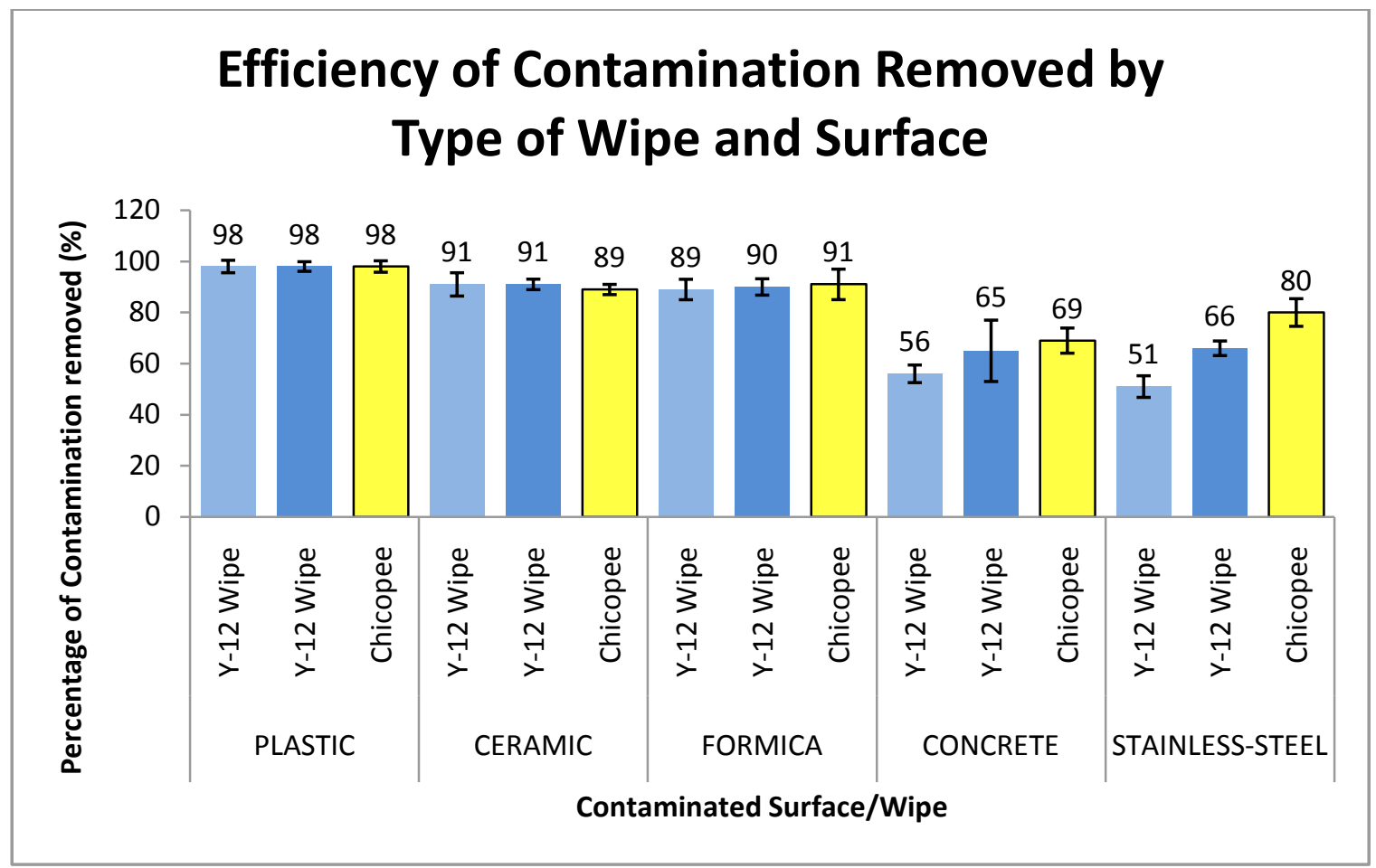

Figure 40 Chart of efficiency of contamination removal per type of wipe used and surface, respectively. The column bars represented the mean percentage of uranium oxide removed and the error bars the standard deviation (Tables 36 through 40, appendix 9.2)

It can be observed from Figure 40 (the error bars shown represented the standard deviation values from tables 36 through 40 , appendix 9.2), that there was a decrease in the amount of contamination removed from the surface of plastic to the ceramic. The plastic (PVC) surfaces are hydrophobic and the contaminant is an oxidized metal (uranium oxide which is hydrophilic). This difference indicates that the adhesion work between the contaminant and the surface is less than the adhesion work between ceramic surfaces (hydrophilic) and the contaminant (uranium oxide, hydrophilic also). As a result, 
the detachment force necessary to remove a contaminant from a surface of PVC is less than the detachment force necessary to remove a contaminant from a surface of ceramic. This difference can be appreciated by the amount of contamination removed from the PVC surface as compared to the ceramic surface. Also, Formica surfaces show the same tendency as ceramic, the amount of contamination removed was higher in PVC than in ceramic and Formica. For the surfaces of concrete and stainless steel, the main factor that affected the process of removal of the contaminant was the surface roughness. But, it can also be observed that wipe 2 (Chicopee Maslin) removed this type of contamination on rough surfaces better. This was expected to occur due to the nature of wipe 2 , which has a surfactant attached to its microfibers, thus allowing it to reach deeper areas on the surfaces and remove the contamination. The values presented in Figure 40 and 41 where as a result of five replications per wipe used on each surface, fifteen replications per surface, representing a total of 75 surfaces scanned.

The average counts per two minutes on the contaminated samples were of $441 \pm 21$. The precision determined by 2 minutes counting were of $\pm 4.76 \% \approx \pm 5 \%$. This value is a good indicator of reproducibility of the measurements made to determinate the amount of contamination removed.

\subsection{Quantification of the contamination by radiation detection: gamma disintegration mode}

The quantification of the contamination by this method was carried out to evaluate the influence of the surface roughness, the surface type, the particle size of the contaminant and their mutual interaction in the efficiency of contamination removed. First, all the 
analytical procedures presented in section 4.5 were calculated to create a radioactive contamination in particle sizes 200 and 400 mesh; the efficiency of the detectors used and the amount of radioactivity necessary to obtain a good sample to noise ratio was then calculated. The surfaces were then contaminated and decontaminated, the data collected was processed using a factorial model and the results analyzed.

\subsubsection{Estimated ratio of $\mathrm{SnCl}_{2}$ to $\mathrm{TcO}_{4}^{-}$ions}

Following the method presented in section 4.1.1 and assuming starting the experiment with an activity of $\mathrm{A}=1 \mathrm{mCi}$, the number of radioactive atoms is:

$\mathrm{N}\left(\mathrm{TcO}_{4}^{-}\right)=\frac{\mathrm{A}}{\lambda}=\frac{37 \times 10^{6}}{\frac{\ln 2}{6.02}} \times 3600=1.156 \times 10^{12}$ radioactive atoms

And the number of moles is:

$\mathrm{n}\left(\mathrm{TcO}_{4}{ }^{-}\right)=\frac{\mathrm{N}\left(\mathrm{TcO}_{4}^{-}\right)}{\mathrm{N}_{\mathrm{A}}}=\frac{1.56 \times 10^{12}}{6.022 \times 10^{23}}=1.92 \times 10^{-12} \mathrm{~mol}$

The concentration of technetium pertechnetate $\left(\mathrm{TcO}_{4}{ }^{-}\right)$is the number of moles divided by the volume of the solution of technetium pertechnetate $\left(\mathrm{TcO}_{4}{ }^{-}\right)$which was $0.4 \mathrm{ml}$, then:

$\mathrm{C}\left(\mathrm{TcO}_{4}^{-}\right)=\frac{1.92 \times 10^{-12} \mathrm{~mol}}{1 \times 10^{-3} \mathrm{l}}=1.92 \times 10^{-9} \mathrm{M}$

On the other side, approximately 240 milligrams of stannous chloride II in anhydrous form was dissolved in $100 \mathrm{ml}$ of $4 \mathrm{M} \mathrm{HCl}$. The number of moles of $\mathrm{SnCl}_{2}$ in the solution is then: 


$$
\mathrm{n}\left(\mathrm{SnCl}_{2}\right)=\frac{\mathrm{m}\left(\mathrm{SnCl}_{2}\right)}{\mathrm{M}\left(\mathrm{SnCl}_{2}\right)}=\frac{0.240 \mathrm{~g}}{(118.71+2 * 35.45) \mathrm{g} / \mathrm{mol}}=\frac{0.240 \mathrm{~g}}{189.61 \mathrm{~g} / \mathrm{mol}}=1.2610^{-3} \mathrm{~mol}
$$

And the concentration is:

$$
\mathrm{n}\left(\mathrm{SnCl}_{2}\right)=\frac{1.2610^{-3} \mathrm{~mol}}{5110^{-1} 1}=1.26 \times 10^{-2} \mathrm{M}
$$

Finally, 10 microliters were extracted from this solution and extended to $5 \mathrm{ml}$ (with a saline solution at $0.9 \% \mathrm{NaCl}$ ) to a final concentration of:

$$
\mathrm{C}\left(\mathrm{SnCl}_{2}\right)=\frac{\left[\mathrm{C}\left(\mathrm{SnCl}_{2}\right)\right] \mathrm{V}_{\mathrm{o}}}{\mathrm{V}_{\mathrm{f}}}=\frac{1.26 \times 10^{-2} \frac{\mathrm{mol}}{1} 10^{-6} \mathrm{l}}{5 \times 10^{-3} 1}=2.53 \times 10^{-5} \mathrm{M}
$$

Finally:

$$
\frac{\mathrm{C}\left(\mathrm{SnCl}_{2}\right)}{\mathrm{C}\left(\mathrm{TcO}_{4}^{-}\right)}=\frac{2.53 \times 10^{-5}}{1.92 \times 10^{-9}}=1.3184 \times 10^{4}=13184
$$

The literature support that the concentration of the $\mathrm{Sn}+2$ ions should be at least 1.5 times the concentration of $\mathrm{TcO}_{4}^{-}$ions [16]. The experiment conducted showed that the concentrations used were above this number, so it is expected to have an almost a $100 \%$ reduction of the $\mathrm{Tc}^{99 \mathrm{~m}}$ ions.

The calculations presented above were standardized each time it was required for the preparation of the contamination (radioactive resins), then expecting the reduction of the technetium to later attach to the Dowex 50Wx2 resin. 


\subsubsection{Estimated initial activity to obtain a good sample to noise ratio}

In section 4, it was estimated that the mass of resin per surface was approximately $13 \mathrm{mg}$; then, the activity per surface, $\mathrm{A}_{\mathrm{f}}$, corresponding to a mass of $\mathrm{m}_{\mathrm{f}}=13 \mathrm{mg}$ of resin is:

$$
A_{f}=\frac{A_{i} m_{f}}{m_{i}}=\frac{3710^{6} * 60 \mathrm{dpm} * 0.013 \mathrm{~g}}{1 \mathrm{~g}}=2.886 \times 10^{7} \mathrm{dpm}
$$

This is the estimated amount of activity per minute per surface, but a 24 hour decay time was necessary to let the contamination dry, thus:

$$
\mathrm{A}=\mathrm{A}_{\mathrm{f}} \mathrm{e}^{-\lambda \mathrm{t}}=2.88610^{7} \exp (-\ln (2) / 6.02 * 24)=1.8210^{6} \mathrm{dpm}
$$

An initial activity of $1.2 \mathrm{mCi}$ per resin vial was used for each $10 \mathrm{ml}$ of ethanol (Table 10 ).

The activity per $10 \mathrm{ul}$ of solution extracted was multiplied by twenty (the number of drops placed on the surfaces) and expressed in disintegrations per minutes (Table 10). The final activity (dpm) was corrected to the time each instrument was used to record their activity values on the surfaces. A summary of these calculations is presented in Table 10.

Finally, twenty-two surfaces were spiked with the mentioned drops and their activity measured (Table 9 and Appendix 9.2). The efficiency of the Eberline detector was found to be:

$$
\eta_{S H P}=\frac{O}{R} * 100=\frac{23153.44}{3106807.37} * 100=0.7452 \%
$$

Finally, the observed counts per minutes for the Eberline detector were:

$$
\mathrm{A}_{\mathrm{o}}=\mathrm{A} \times \eta_{\text {detector }}=1.8210^{6} * 0.007452=13564 \mathrm{cpm}
$$


Table 9 Average and standard deviation in counts per minute of 22 surfaces, using the Eberline detector

\begin{tabular}{|c|c|}
\hline Average (cpm) & 23153 \\
\hline STDEV & 1837 \\
\hline CV $(\%)$ & 8 \\
\hline
\end{tabular}

The 13564 counts per minute were large enough to have a large sample-to-background ratio (background levels were of $200 \mathrm{cpm}$ ).

For the Cobra Packard 5003 II, sixteen test tubes were spiked with $10 \mu 1$ droplets of the solution originally prepared. Table 11 summarizes the values obtained.

Table 10 Values obtained to calculated the efficiency of the detectors

\begin{tabular}{|c|c|c|c|}
\hline Instruments & Summary of results & Values & Time measured \\
\hline Dose Calibrator & Initial Activity (mCi) & 1.12 & \\
\hline \multirow{4}{*}{ Atom lab 100} & Initial Volume (ml) & 10 & First day \\
\hline & volume per drop (ul) & 10 & \\
\hline & Activity per drop (mCi) & 0.02 & $10: 47 \mathrm{am}$ \\
\hline & Activity per surface (dpm) & 49728000 & \\
\hline \multirow{3}{*}{$\begin{array}{l}\text { Eberline } \\
\text { detector }\end{array}$} & \multirow{2}{*}{$\begin{array}{l}\text { Time elapsed between } \\
\text { measurements }(\mathrm{hr})\end{array}$} & \multirow[t]{2}{*}{24.08} & Second day \\
\hline & & & 10:52am \\
\hline & Corrected activity (dpm) & 3106807 & \\
\hline \multirow{3}{*}{$\begin{array}{l}\text { Packard } \\
\text { detector }\end{array}$} & \multirow{2}{*}{$\begin{array}{l}\text { Time elapsed between } \\
\text { measurements }(\mathrm{hr})\end{array}$} & \multirow[t]{2}{*}{26.52} & Second day \\
\hline & & & $1: 23 \mathrm{pm}$ \\
\hline & Corrected activity (dpm) & 2347662 & \\
\hline
\end{tabular}

The efficiency of the Cobra detector was found to be:

$$
\eta_{S H P}=\frac{O}{R} * 100=\frac{825314.3552}{2347661.81} * 100=35.15 \%
$$

As a result, the observed counts per minutes for the Cobra detector is:

$$
\mathrm{A}_{\mathrm{o}}=\mathrm{A} \times \eta_{\text {detector }}=1.8210^{6} * 0.35=6.3710^{5} \mathrm{cpm}
$$


The $6.3710^{5} \mathrm{cpm}$ were large enough to have a large sample-to-background ratio (background levels were of $28 \mathrm{cpm}$ ).

Table 11 Cobra Packard detector summary of the measurement

\begin{tabular}{|l|r|}
\hline Average number of counts (cpm) per surface & 825314 \\
\hline STDEV & 84257 \\
\hline CV & 0.10 \\
\hline
\end{tabular}

\subsubsection{Estimated influence of ethanol in the ${ }^{99 \mathrm{~m}}$ Tc-Dowex $50 \mathrm{~W} \times 2$ resin binding}

Approximately one gram of the organic resin Dowel 50Wx8 (50-100 mesh particle size)

was used. The activity of the vial before starting the experiment was $1.8 \mathrm{mCi}$ in $2 \mathrm{ml}$. In $0.4 \mathrm{ml}$, the amount of activity was $360 \mu \mathrm{Ci}$. After mixing all of the components $\left(\mathrm{SNCl}_{2}\right.$, $\mathrm{TcO}_{4}{ }^{-}$and the resin) together, the mixture was agitated for 10 minutes. Then, the mixture was centrifuged and the supernatant was extracted from the sample. The supernatant was measured as well as the remaining resin.

The efficiency of the labeling process was estimated by:

$$
\eta=\frac{A_{i}-A_{s}}{A_{i}}=\frac{360-75.5}{360}=0.790 \approx 0.8 * 100=80 \%
$$

Where $A_{i}$ is the initial activity of the mixture in micro curie; $A_{s}$ is the activity remained in the supernatant in microcuries.

To determine whether ethanol could break any binding between the ${ }^{99 \mathrm{~m}} \mathrm{Tc}$ and the resin, the resin was mixed with ethanol and centrifuged; the efficiency was determined as:

$$
\eta=\frac{A_{\text {ir }}-A_{\text {se }}}{A_{\text {ir }}}=\frac{291-11.2}{291}=0.96 * 100=96 \%
$$


Where $A_{i r}$ is the activity present in the resin with ethanol and $A_{\text {se }}$ is the activity in the supernatant due to ethanol, both expressed in micro curies.

The Tc-99m attaches to the resin with an efficiency of $80 \%$ and the use of ethanol does not affect the binding process; $96 \%$ of the activity remained in the resin after being separated via centrifugation. The missing $4 \%$ of activity might be due to ${ }^{99 \mathrm{~m}} \mathrm{Tc}$ ions present on the solution that was not bound to the resin.

\subsubsection{Roughness \& particle size influence on the efficiency of loose contamination removal: Model population \& data processing}

The technetium $99 \mathrm{~m}\left({ }^{99 \mathrm{~m}} \mathrm{Tc}\right)$ isotope was received in a vial with an activity $\mathrm{A}=5.7 \mathrm{mCi}$ in a volume of $2 \mathrm{ml}$. Two samples, $400 \mu \mathrm{l}$ each, were extracted from the vial containing the isotope. The following table shows the activities measured by the volume extracted and by particle size of the contaminant.

Table 12 Initial activity used for labeling the particles in a 200 and 400 mesh

\begin{tabular}{|c|c|c|}
\hline Volume (ul) & Size (mesh) & Activity $(\boldsymbol{\mu C i})$ \\
\hline 400 & 200 & 924 \\
\hline 400 & 400 & 916 \\
\hline
\end{tabular}

Then, as explained in section 4.5.4, the efficiency of the labeling of Tc-99m to the Dowex 50Wx2 resin was determined. The numbers presented in Table 13 are the activity in the supernatant and the resin.

Table 13 Activities measured in the supernatant and the solid after labeling

\begin{tabular}{|l|c|c|}
\hline & 200 Mesh Activity $(\boldsymbol{\mu C} \mathbf{C})$ & $\mathbf{4 0 0}$ Mesh Activity $(\boldsymbol{\mu C} \mathbf{)}$ \\
\hline Initial & 924 & 916 \\
\hline Supernatant & 193 & 135 \\
\hline Resin & 651 & 723 \\
\hline
\end{tabular}


The values reported in Table 13 were used to calculate the efficiency of the labeling process, which is the amount of radioactive Tc-99m bonded to the resin.

$$
\begin{aligned}
& \eta_{200}=\frac{A_{i 200}-A_{s 200}}{A_{i 200}}=\frac{924-193}{924}=0.791 \stackrel{\text { yields }}{\longrightarrow} 79.1 \% \\
& \eta_{400}=\frac{A_{i 400}-A_{s 400}}{A_{i 400}}=\frac{916-135}{916}=0.852 \stackrel{\text { yields }}{\longrightarrow} 85.2 \%
\end{aligned}
$$

where $A_{i 200}$ and $A_{i 400}$ (in microcuries) were the initial activities of Tc-99m in the solutions with particles of mesh sizes 200 and 400 respectively; and $A_{s 200}$ and $A_{s 400}$ were the activities of Tc-99m in the supernatant with particles of mesh sizes 200 and 400, respectively. Additionally, a $10 \mu 1$ droplet of contaminant solution was added to each of twelve test tubes (section 4.1.4, and table 14).

Table 14 Test of homogeneity of by particle size

\begin{tabular}{|c|c|c|}
\hline No. & 400mesh & 200mesh \\
\hline 1 & 65358 & 38966 \\
\hline 2 & 89330 & 32946 \\
\hline 3 & 36180 & 34274 \\
\hline 4 & 21404 & 34870 \\
\hline 5 & 25614 & 38998 \\
\hline 6 & 27022 & 40144 \\
\hline 7 & 31644 & 39218 \\
\hline 8 & 39394 & 37358 \\
\hline 9 & 43326 & 39182 \\
\hline 10 & 63584 & 34892 \\
\hline 11 & 54248 & 38358 \\
\hline 12 & 22708 & 35130 \\
\hline average & 43318 & 37028 \\
\hline stdev & 20932 & 2444 \\
\hline
\end{tabular}


A t-test was then performed to determine if there was a significant difference between mean values derived for each sample set. Finally, $t_{o}=1.03$ and $t_{0.025,18}=2.07$. As $\left(t_{0} \geq t_{0.025,18}\right)$, (Figure 41 , data provided by table 14 ) the null hypothesis could not be rejected, concluding that the difference in the means between solutions with particle mesh sizes of 200 and 400 was not significant, and thus can be considered the same. The experiment was conducted following the procedure described in section 4.5.10 As a result, the amount of contamination before and after cleaning the surface was obtained in kilo counts per minute.

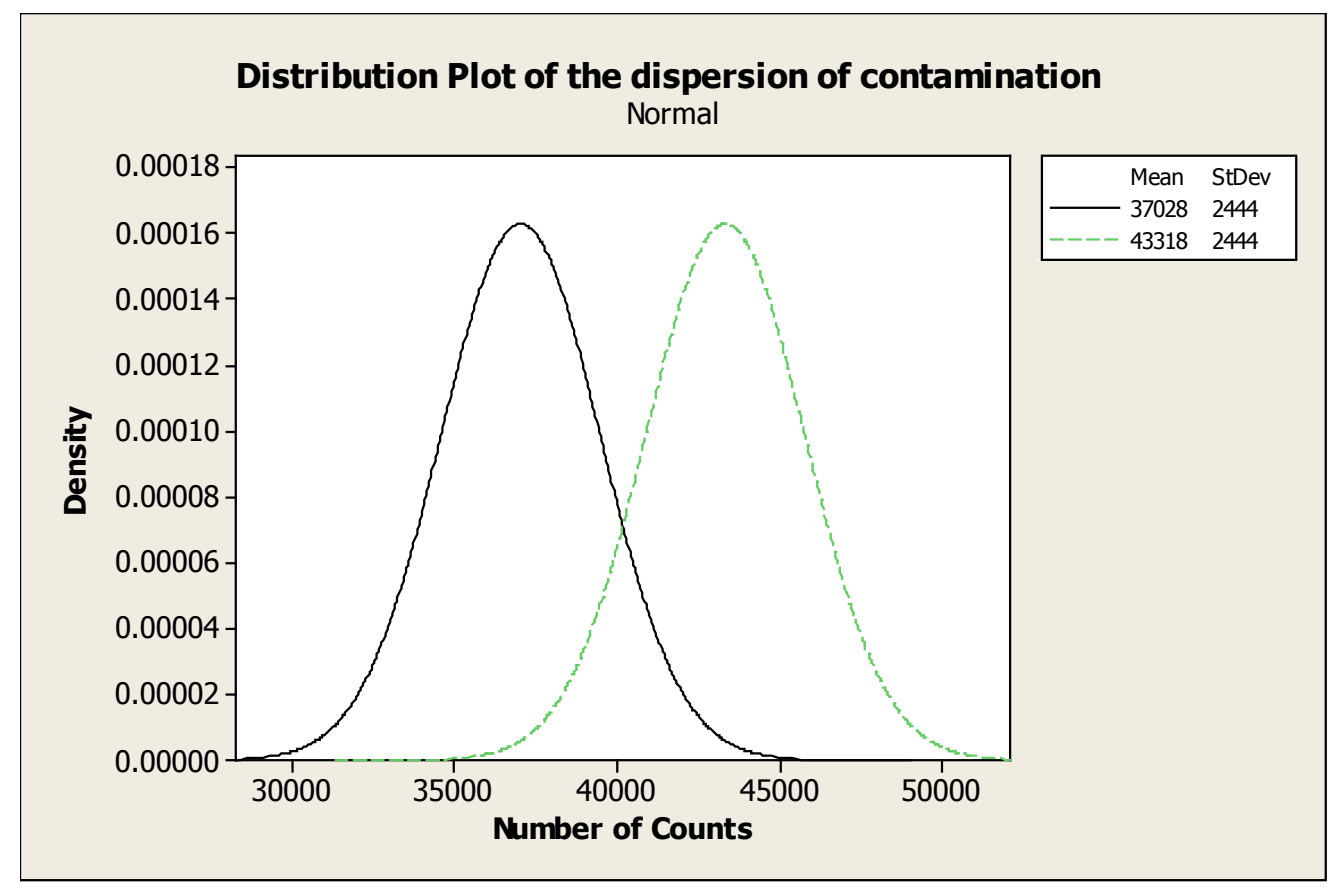

Figure 41 Normal distribution plot of particles of 200 and 400 mesh (black and dash green line, respectively)

Table 15 presents a summary of all the information gathered to evaluate the efficiency of loose contamination removal by direct counting of the contamination removed from the surface. 
In Table 15, the first column is the order in which the surfaces were decontaminated; the second column represents the surface number. Columns 3 to 5 are the factors used on each factorial combination. In column 3, W2 refers to wipe 2, and W1 refers to wipe 1. In column 5, 200M refers to the decontamination made with particle size of 200 meshes, and $400 \mathrm{M}$ refers to decontamination of particle size of 400 meshes. Column 6 shows the number of kilo counts per min $(\mathrm{kcpm})$ on the surface before wiping at the clock time given in column 8 . Similarly, column 7 shows kcpm on the surface after wiping at the time in column 9.

Table 15 Populated matrix with the experimental values obtained, following the order presented in Table 2.

\begin{tabular}{|c|c|c|c|c|c|c|c|c|}
\hline Order & No & $\mathbf{A}$ & $\mathbf{B}$ & $\mathbf{C}$ & $\mathbf{k C P M}$ & $\mathbf{k C P M}$ & Time in & Time out \\
\hline 3 & 1 & W2 & Concrete & $200 \mathrm{M}$ & 8.57 & 8.06 & $10: 10$ & $10: 12$ \\
\hline 17 & 2 & W1 & Concrete & $200 \mathrm{M}$ & 8.73 & 8.28 & $11: 02$ & $11: 04$ \\
\hline 14 & 3 & W2 & PVC & $200 \mathrm{M}$ & 11.62 & 3.36 & $10: 52$ & $10: 54$ \\
\hline 11 & 4 & W2 & Concrete & $400 \mathrm{M}$ & 9.61 & 5.75 & $10: 41$ & $10: 43$ \\
\hline 6 & 5 & W1 & PVC & $200 \mathrm{M}$ & 14.58 & 4.93 & $10: 22$ & $10: 24$ \\
\hline 13 & 6 & W1 & Concrete & $400 \mathrm{M}$ & 9.06 & 5.3 & $10: 48$ & $10: 50$ \\
\hline 20 & 7 & W2 & PVC & $400 \mathrm{M}$ & 13.74 & 3.22 & $11: 13$ & $11: 15$ \\
\hline 16 & 8 & W1 & PVC & $400 \mathrm{M}$ & 15.66 & 3.14 & $10: 58$ & $11: 00$ \\
\hline 8 & 9 & W2 & Concrete & $200 \mathrm{M}$ & 8.94 & 8.32 & $10: 30$ & $10: 32$ \\
\hline 24 & 10 & W1 & Concrete & $200 \mathrm{M}$ & 6.75 & 6.18 & $11: 26$ & $11: 28$ \\
\hline 18 & 11 & W2 & PVC & $200 \mathrm{M}$ & 10.78 & 1.462 & $11: 06$ & $11: 07$ \\
\hline 22 & 12 & W2 & Concrete & $400 \mathrm{M}$ & 11.65 & 5.65 & $11: 20$ & $11: 21$ \\
\hline 1 & 13 & W1 & PVC & $200 \mathrm{M}$ & 15.44 & 4.93 & $10: 04$ & $10: 06$ \\
\hline 19 & 14 & W1 & Concrete & $400 \mathrm{M}$ & 10.19 & 5.39 & $11: 09$ & $11: 11$ \\
\hline 9 & 15 & W2 & PVC & $400 \mathrm{M}$ & 15.71 & 3.91 & $10: 33$ & $10: 35$ \\
\hline 7 & 16 & W1 & PVC & $400 \mathrm{M}$ & 17.5 & 5.39 & $10: 26$ & $10: 28$ \\
\hline 15 & 17 & W2 & Concrete & $200 \mathrm{M}$ & 8.74 & 7.86 & $10: 55$ & $10: 57$ \\
\hline 5 & 18 & W1 & Concrete & $200 \mathrm{M}$ & 8.37 & 7.65 & $10: 18$ & $10: 20$ \\
\hline 21 & 19 & W2 & PVC & $200 \mathrm{M}$ & 13.46 & 3.73 & $11: 16$ & $11: 18$ \\
\hline 4 & 20 & W2 & Concrete & $400 \mathrm{M}$ & 12.06 & 7.67 & $10: 14$ & $10: 16$ \\
\hline 23 & 21 & W1 & PVC & $200 \mathrm{M}$ & 10.78 & 2.91 & $11: 23$ & $11: 25$ \\
\hline 10 & 22 & W1 & Concrete & $400 \mathrm{M}$ & 13.08 & 8.39 & $10: 37$ & $10: 39$ \\
\hline 2 & 23 & W2 & PVC & $400 \mathrm{M}$ & 17.23 & 5.58 & $10: 07$ & $10: 09$ \\
\hline 12 & 24 & W1 & PVC & $400 \mathrm{M}$ & 16.17 & 4.08 & $10: 44$ & $10: 46$ \\
\hline
\end{tabular}


Before processing the data, the first step was to subtract the background measured at the beginning of the experiment. Since Tc-99m has a short half life $(6.02 \mathrm{~h})$ the observed count rates were corrected for the decay and all count rates were normalized to the same time (see Appendix 9.4). Using the corrected counts, the efficiency of decontamination removal was obtained (see Appendix 9.4 and Table 16). It can be observed, in Table 16, how the efficiency of loose contamination removal changes when changing the wipe used, the surface roughness, and the particle size of the contaminant, each time denoted with letters a, b, and c, respectively.

The efficiency of contamination removal was also characterized by quantifying the amount of contamination collected by the wipes (section 4.5.8). This method estimated the total number of counts per minute on each surface as the average of the number of counts per minute in a drop of $10 \mu 1$ times the number of drops placed on a surface (Table 17). A summary of the values obtained is presented in Table 18. The values of efficiency of contamination removal presented on Table 18 are calculated based on the contamination gathered by the wipes.

\subsubsection{Data processing and analysis}

The values of efficiency of contamination removal (presented in Tables 16 and 18) were analyzed following a factorial model algorithm. Factorial models are a useful tool because they help to identify which of the factors (wipes, surface types or particle size), and which of the interactions among the factors involved in the process create a significant variation in the efficiency of contamination removal. Additionally, a factorial 
model generates a linear regression equation which fits the observations, and it helps to explain the variability that might occur in the efficiency of contamination removal.

Table 16 Results expressed as efficiency of contamination removal

\begin{tabular}{|c|c|c|}
\hline Surface No. & Factorial & $\begin{array}{l}\text { Efficiency of Contamination Removal } \\
(\%)\end{array}$ \\
\hline 1 & -1 & 5.70 \\
\hline 2 & $\mathrm{a}$ & 4.89 \\
\hline 3 & $\mathrm{~b}$ & 71.98 \\
\hline 4 & $\mathrm{c}$ & 40.63 \\
\hline 5 & $\mathrm{ab}$ & 66.80 \\
\hline 6 & $\mathrm{ac}$ & 42.03 \\
\hline 7 & bc & 77.39 \\
\hline 8 & abc & 80.71 \\
\hline 9 & -1 & 6.71 \\
\hline 10 & $\mathrm{a}$ & 8.30 \\
\hline 11 & $\mathrm{~b}$ & 87.73 \\
\hline 12 & $\mathrm{c}$ & 52.14 \\
\hline 13 & $a b$ & 68.67 \\
\hline 14 & ac & 47.67 \\
\hline 15 & $\mathrm{bc}$ & 75.80 \\
\hline 16 & $a b c$ & 69.73 \\
\hline 17 & -1 & 9.91 \\
\hline 18 & $\mathrm{a}$ & 8.42 \\
\hline 19 & $\mathrm{~b}$ & 73.07 \\
\hline 20 & $\mathrm{c}$ & 36.65 \\
\hline 21 & $a b$ & 74.02 \\
\hline 22 & $\mathrm{ac}$ & 36.06 \\
\hline 23 & $\mathrm{bc}$ & 68.13 \\
\hline 24 & $a b c$ & 75.43 \\
\hline
\end{tabular}

Table 17 Estimation of the amount of contamination per surface

\begin{tabular}{|l|l|}
\hline Initial Amount of Contamination & \\
\hline Average of counts in $10 \mu \mathrm{l}(\mathrm{cpm})$ & 40173 \\
\hline Number of drops per surface & 20 \\
\hline Estimated amount of contamination per surface $(\mathrm{cpm})$ & 803457 \\
\hline
\end{tabular}


Table 18 Data of the amount of loose contamination removal calculated using equation 2.

\begin{tabular}{|c|c|c|c|}
\hline $\begin{array}{l}\text { Surface } \\
\text { No. }\end{array}$ & Factorial & $\begin{array}{l}\text { Contamination on } \\
\text { Wipes (cpm) }\end{array}$ & $\begin{array}{l}\text { Efficiency of } \\
\text { Contamination Removal } \\
(\%)\end{array}$ \\
\hline 1 & -1 & 48070 & 5.98 \\
\hline 2 & $\mathrm{a}$ & 49006 & 6.10 \\
\hline 3 & $\mathrm{~b}$ & 635990 & 79.16 \\
\hline 4 & $\mathrm{ab}$ & 593804 & 73.91 \\
\hline 5 & $\mathrm{c}$ & 259110 & 32.25 \\
\hline 6 & $\mathrm{ac}$ & 243988 & 30.37 \\
\hline 7 & $\mathrm{bc}$ & 556552 & 69.27 \\
\hline 8 & $\mathrm{abc}$ & 567944 & 70.69 \\
\hline 9 & -1 & 53300 & 6.63 \\
\hline 10 & $\mathrm{a}$ & 48724 & 6.06 \\
\hline 11 & $\mathrm{~b}$ & 709694 & 88.33 \\
\hline 12 & $\mathrm{ab}$ & 601324 & 74.84 \\
\hline 13 & $\mathrm{c}$ & 366136 & 45.57 \\
\hline 14 & $\mathrm{ac}$ & 285944 & 35.59 \\
\hline 15 & $\mathrm{bc}$ & 646750 & 80.50 \\
\hline 16 & $\mathrm{abc}$ & 597022 & 74.31 \\
\hline 17 & -1 & 52060 & 6.48 \\
\hline 18 & $\mathrm{a}$ & 44334 & 5.52 \\
\hline 19 & $\mathrm{~b}$ & 664256 & 82.67 \\
\hline 20 & $\mathrm{ab}$ & 562932 & 70.06 \\
\hline 21 & $\mathrm{c}$ & 233450 & 29.06 \\
\hline 22 & $\mathrm{ac}$ & 242312 & 30.16 \\
\hline 23 & $\mathrm{bc}$ & 632290 & 78.70 \\
\hline 24 & $a b c$ & 693052 & 86.26 \\
\hline
\end{tabular}

The data, as presented in Tables 16 and 18, was used to find the regression coefficients (section 4.5.9). Then, a regression test of the regressor coefficients was made following an ANOVA method. The software used to process the data was MINITAB. After processing the data presented in Table 19, the following table was obtained. 
Table 19 Estimated effect and coefficients of the factorial model for the Efficiency of Contamination Removal using data from Table 14

\begin{tabular}{|l|c|c|l|c|c|}
\hline Term & Effect & Coef & SE Coef. & T & P-value \\
\hline Constant & & 49.525 & 1.149 & 43.12 & $<\mathbf{0 . 0 1}$ \\
\hline Wipe & -1.926 & -0.963 & 1.149 & -0.84 & 0.41 \\
\hline Surface & 49.197 & 24.598 & 1.149 & 21.42 & $<\mathbf{0 . 0 1}$ \\
\hline Particle size & 18.014 & 9.007 & 1.149 & 7.84 & $<\mathbf{0 . 0 1}$ \\
\hline Wipe*Surface & -1.197 & -0.599 & 1.149 & -0.52 & 0.61 \\
\hline Wipe*Particle size & 2.074 & 1.037 & 1.149 & 0.90 & 0.38 \\
\hline Surface*Particle size & -17.194 & -8.597 & 1.149 & -7.48 & $<\mathbf{0 . 0 1}$ \\
\hline Wipe*Surface*Particle size & 2.565 & 1.282 & 1.149 & 1.12 & 0.28 \\
\hline
\end{tabular}

Table 19 provides an estimated value of the contribution of the factors (wipe, surface roughness, and particle size of the contaminant) to the efficiency on contamination removal. Also, it provides the estimated value effect of the interactions of the mentioned factors. Note that when the P-value (last column of Table 19) is very small $\mathrm{P}<0.01$ (black highlighted), it means that this effect or interaction effect rejects the null hypothesis (as it is stated in section 4.5.9), thus concluding that this factor effect or interaction effect is significant to the value of efficiency of contamination removal. In consequence, from Table 10 and Pareto chart shown in Figure 42, it can be observed that the surface roughness, the particle size of the contaminant and their mutual interaction significantly changes the efficiency of contamination removal, no matter which wipe was used. In addition, the wipes involved in the decontamination procedure (wipe 1 and wipe 2) remove similar amounts of contamination (P-value Table 19). 


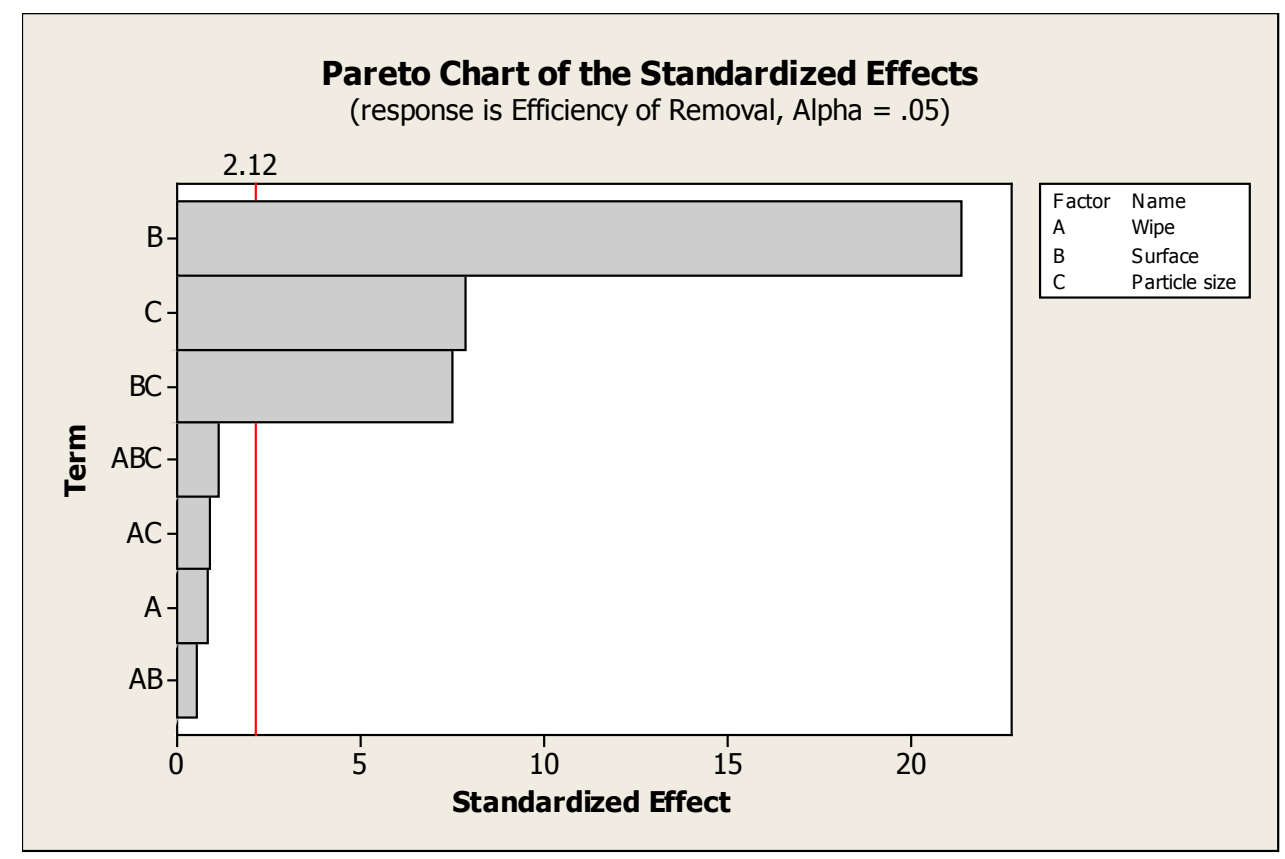

Figure 42 Pareto chart showing the significant effects within the empirical model.

Table 20 Analysis of Variance for the Efficiency of Contamination Removal obtained with data from Table 14

\begin{tabular}{|l|c|c|c|c|c|c|}
\hline Source & DF & Seq SS & Adj SS & MS & F & P-value \\
\hline Main Effects & 3 & 16491.3 & 16491.3 & 5497.1 & 173.63 & $<\mathbf{0 . 0 1}$ \\
\hline 2-Way interactions & 3 & 1808.1 & 1808.1 & 602.7 & 19.04 & $<\mathbf{0 . 0 1}$ \\
\hline 3-Way interactions & 1 & 39.5 & 39.5 & 39.4 & 1.25 & 0.28 \\
\hline Residual & 16 & 506.6 & 506.6 & 31.6 & & \\
\hline Pure error & 16 & 506.6 & 506.6 & 31.6 & & \\
\hline Total & 23 & 18845.4 & & & & \\
\hline
\end{tabular}

Finally, Table 20 presents a summary of the ANOVA method used to test the estimated effects within the regression equation. From Table 20, in can be observed that the first and second order interaction are governing the regression equation (P-value Table 20), this means that the polynomial that fits the data had a significant two way interaction, and the contour plot describing the process was plotted a parabolic shape was appreciated.

Based on the values obtained in Table 19 (third column left to right), equation 3 can be rewritten as: 
$\mathrm{y}=49.25-0.96 \mathrm{x}_{1}+24.59 \mathrm{x}_{2}+9 \mathrm{x}_{3}-0.59 \mathrm{x}_{1} \mathrm{x}_{2}+1.04 \mathrm{x}_{1} \mathrm{x}_{3}-8.59 \mathrm{x}_{2} \mathrm{x}_{3}+1.282 \mathrm{x}_{1} \mathrm{x}_{2} \mathrm{x}_{3}$

Where $\mathrm{y}$ is the predicted efficiency of loose contamination removal, and $\mathrm{x}_{1}, \mathrm{x}_{2}$ and $\mathrm{x}_{3}$ are the coded variables that represent the wipe used, the surface roughness and the particle size of the contaminant, respectively.

Equation 31 can explain the variability of the observations (efficiency of loose contamination removal) $97.31 \%$ (Table 21 ) of the times and predict a new observation with a precision of $93.95 \%$ (Table 21$)$.

Table 21 Regression fit estimators

\begin{tabular}{|l|c|}
\hline Regression fit & Value \\
\hline $\mathrm{S}$ & 5.6 \\
\hline $\mathrm{R}-\mathrm{Sq}$ & $97.3 \%$ \\
\hline $\mathrm{PRESS}$ & 1139.8 \\
\hline $\mathrm{R}-\mathrm{Sq}($ pred$)$ & $93.9 \%$ \\
\hline $\mathrm{R}-\mathrm{Sq}($ adj $)$ & $96.1 \%$ \\
\hline
\end{tabular}

Also, equation 31 can be modeled graphically in order to better understand its importance. A 2D representation of equation 31 is presented in Figure 43. Figure 43 represented the prediction values of efficiency of loose contamination removal for a contaminant of particle size of 400 and 200 mesh, respectively, when the wiped used varies ( 1 means wipe 1 and -1 means wipe 2$)$ and the roughness of the surface (1 means plastic and -1 means concrete) in which the contaminant is presented also varies. It can be observed that variations in the wipe used do not affect the efficiency of contamination removal for a certain value of surface roughness (see how all the color strips are horizontal and parallel each other). The spectrum of colors used represents variations in 
the efficiency of loose contamination removal (dark blue means less and dark green means more contamination removed, respectively).

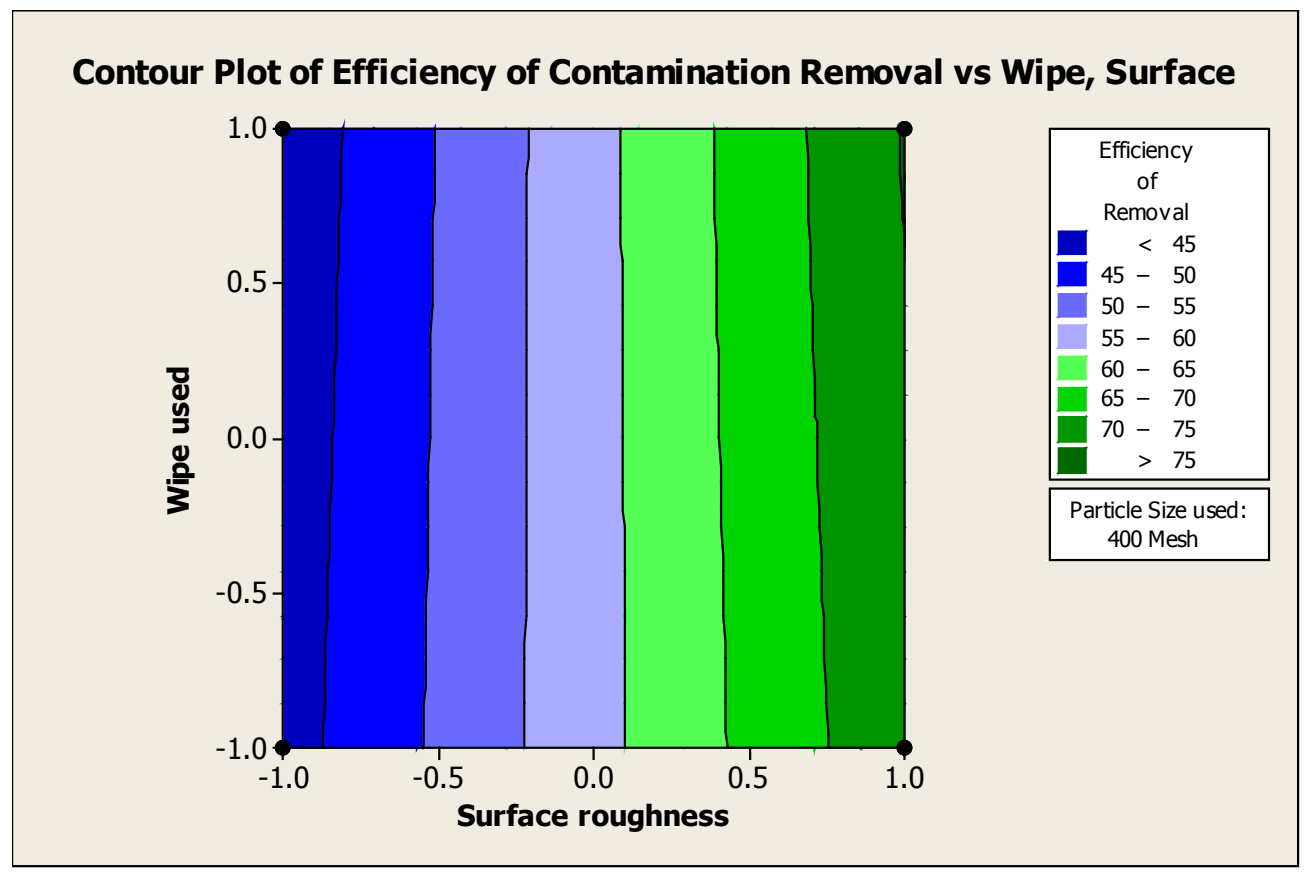

Figure 43 Contour plot of the Efficiency of loose contamination removal versus the wiped used, and the surface roughness for particles of 400 mesh

However, when plotted, the two significant factors (particle size of the contaminant and roughness of the surface), the spectrum changes completely (Figure 44), taking a parabolic shape because of the significance of the interaction particle size of the contaminant and surface roughness (as depicted by the ANOVA Table 20, small P-value for two way interactions). The efficiency of contamination removal significantly changes when the roughness of the surface varies from concrete to PVC (Figure 44). Also, the particle size of the contaminant plays a significant role in the process of contamination removal; for particles between $37-74 \mu \mathrm{m}$, the amount of contamination collected by wiping procedures is greater than for particles of 74-149 $\mu \mathrm{m}$ (Figure 44). 


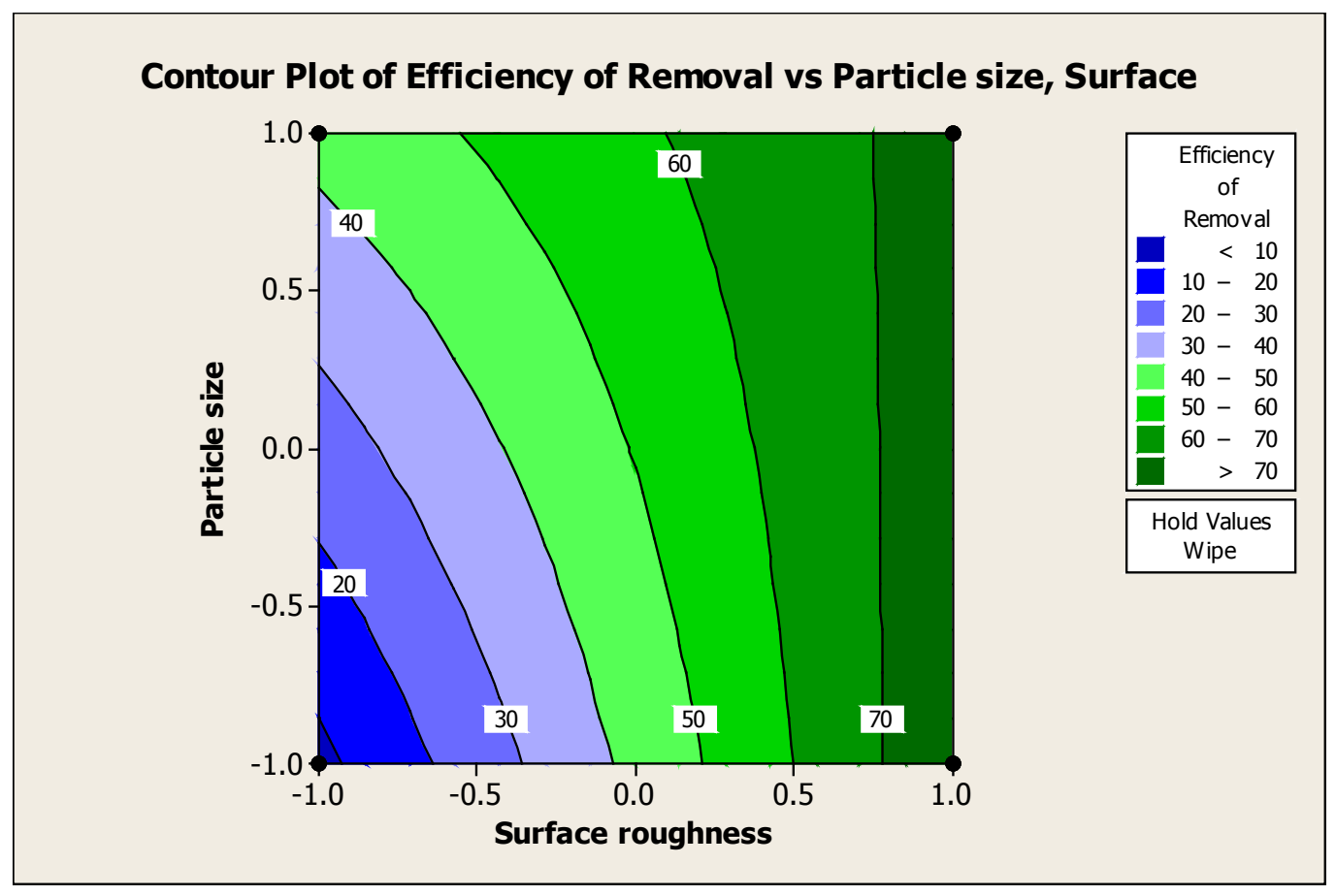

Figure 44 Contour plot of the efficiency of loose contamination removal, particle size of the contaminant(y axis) versus roughness of the surfaces ( $\mathrm{x}$ axis) for wipe 2

An additional important observation from Figure 44 is that when the particle size was on the order of 74-149 $\mu \mathrm{m}$ and the decontaminated surfaces was concrete, there was a significant negative effect to the efficiency of loose contamination removal, in which this efficiency decays to values of $10 \%$ or less. Similarly, for wipe 1 , the graph will take a similar form because both factors are not statistically different (P-value on Table 19). The model adequacy checking of the empirical model developed is presented in Appendix 9.4.1. The normal plot of the residual showed not deviation from the normality, the residual are randomly distributed and overall the model is adequate to describe the process.

Analogously, after processing the values presented in Table 18 (those were obtained by the indirect method presented in section 4.5.9), Tables 22 and 23 were obtained. From 
there, it can be seen that the significant factors and interactions were the surface roughness, the particle size of the contaminant, and their interaction (Table 22, P-value column and Pareto chart Figure 45), respectively. In Appendix 9.4.2 is presented the graphs of normality plot of the residuals and the residuals versus fitted value, particle size of the contaminant and surface roughness, respectively.

Similarly, using the values from Table 22, the regression equation takes the form:

$\mathrm{y}=48.68-1.69 \mathrm{x}_{1}+28.71 \mathrm{x}_{2}+6.54 \mathrm{x}_{3}-0.68 \mathrm{x}_{1} \mathrm{x}_{2}+1.03 \mathrm{x}_{1} \mathrm{x}_{3}-7.31 \mathrm{x}_{2} \mathrm{x}_{3}+1.81 \mathrm{x}_{1} \mathrm{x}_{2} \mathrm{x}_{3}$

Table 22 Estimated effects and coefficients of the factorial model for the efficiency of contamination removal using equation 2

\begin{tabular}{|l|c|c|c|c|c|}
\hline Term & Effect & Coef & SE Coef. & T & P-value \\
\hline Constant & & 48.686 & 1.063 & 45.8 & $<\mathbf{0 . 0 1}$ \\
\hline Wipes & -3.394 & -1.697 & 1.063 & -1.6 & 0.13 \\
\hline Surface & 57.410 & 28.705 & 1.063 & 27.0 & $<\mathbf{0 . 0 1}$ \\
\hline Particle size & 13.079 & 6.54 & 1.063 & 6.2 & $<\mathbf{0 . 0 1}$ \\
\hline Wipes*Surface & -1.365 & -0.683 & 1.063 & -0.6 & 0.53 \\
\hline Wipes*Particle size & 2.066 & 1.033 & 1.063 & 0.9 & 0.35 \\
\hline Surface*Particle size & -14.623 & -7.311 & 1.063 & -6.8 & $<\mathbf{0 . 0 1}$ \\
\hline Wipes*Surface*Particle & 3.624 & 1.812 & 1.063 & 1.7 & 0.11 \\
\hline
\end{tabular}

Table 23 Analysis of Variance for Efficiency of Contamination Removal obtained by using equation 2

\begin{tabular}{|l|c|c|c|c|c|c|}
\hline Source & DF & Seq SS & Adj SS & MS & F & P-value \\
\hline Main effects & 3 & 20871.0 & 20871.0 & 6957.0 & 256.5 & $<\mathbf{0 . 0 1}$ \\
\hline 2-Way interactions & 3 & 1319.7 & 1319.7 & 439.9 & 16.2 & $<\mathbf{0 . 0 1}$ \\
\hline 3-Way interactions & 1 & 78.8 & 78.8 & 78.8 & 2.9 & 0.11 \\
\hline Residual error & 16 & 434.0 & 434.0 & 27.1 & & \\
\hline Pure error & 16 & 434.0 & 434.0 & 27.1 & & \\
\hline Total & 23 & 22703.6 & & & & \\
\hline
\end{tabular}

As before, equation 32 was modeled graphically in order to better understand its importance. A 2D representation of equation 32 is presented in Figure 47. Figure 47 
represented the prediction values of efficiency of loose contamination removal when cleaning with wipe 2 and varies the particle size of the contaminant between 37-74 $\mu \mathrm{m}$ and 74-149 $\mu \mathrm{m}$ ( 1 and -1 , respectively) and the roughness of the surface between PVC and concrete (1 and -1, respectively) in which the contamination is located.

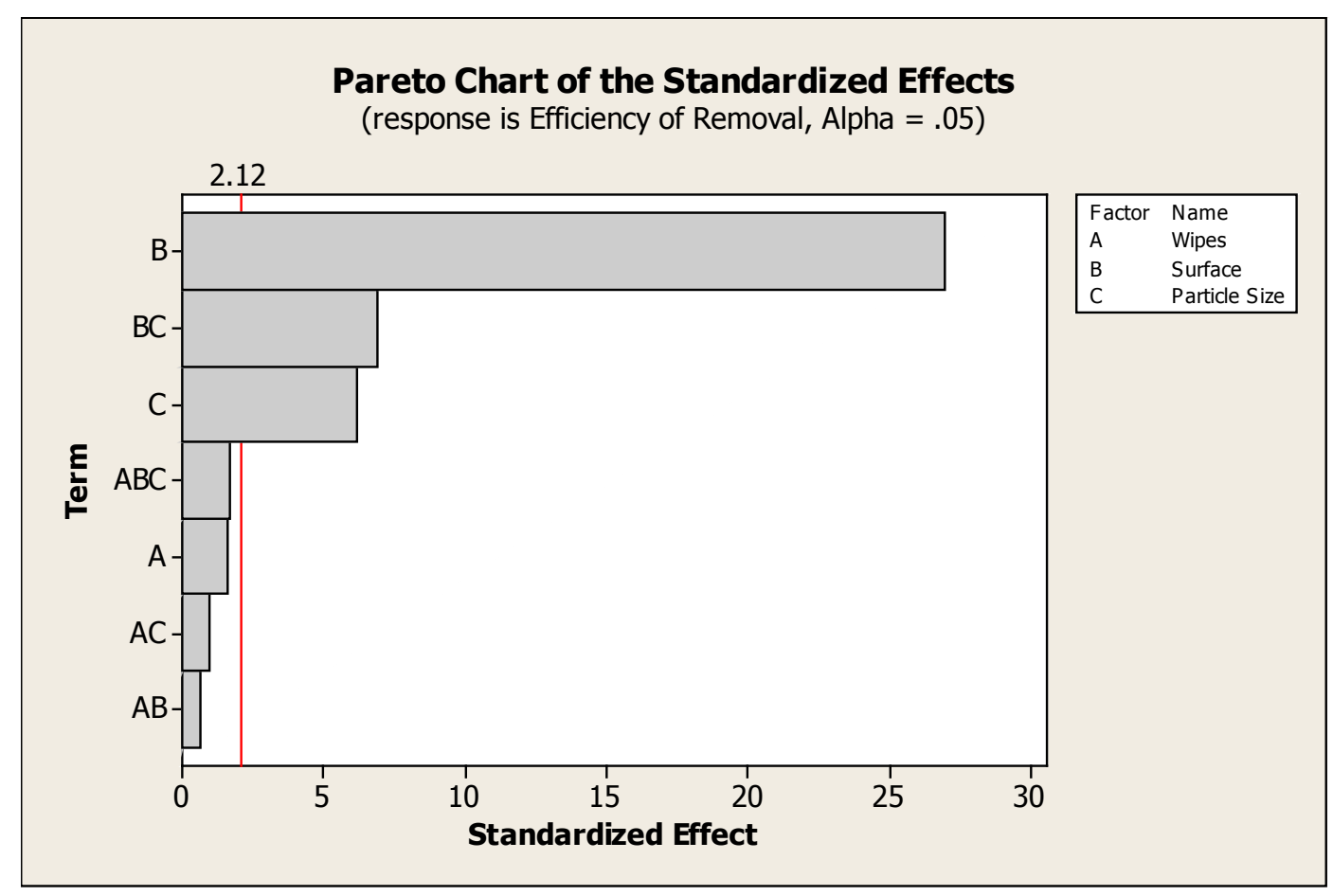

Figure 45 Pareto chart showing the significant effect within the empirical model It can be observed that a variation in the particle size of the contaminant and the roughness of the surface did significantly affect the efficiency of contamination removal, no matter which rag was used to decontaminate the surface. (parabolic shape of the contour line presented in Figures 44 and 46). The spectrum of colors used represents variation in the efficiency of loose contamination removal (dark blue means less and dark green means more contamination removed). 
Table 24 Regression fit estimators

\begin{tabular}{|l|c|}
\hline Regression fit estimators & Value \\
\hline $\mathrm{S}$ & 5.2 \\
\hline R-Sq & $98.1 \%$ \\
\hline PRESS & 976.5 \\
\hline R-Sq(pred) & $95.7 \%$ \\
\hline R-Sq(adj) & $97.3 \%$ \\
\hline
\end{tabular}

Analogously to the evaluation of the contamination removed before, the indirect method to estimate the efficiency of contamination removal accounts for a negative significant effect in the value of efficiency of contamination removal when decontaminated the surface of concrete and the contaminant has a particle size in a range of 74-149 $\mu \mathrm{m}$. At this point, the amount of contamination removed decays to $20 \%$ or less (see dark blue corners on Figures 44 and 46).

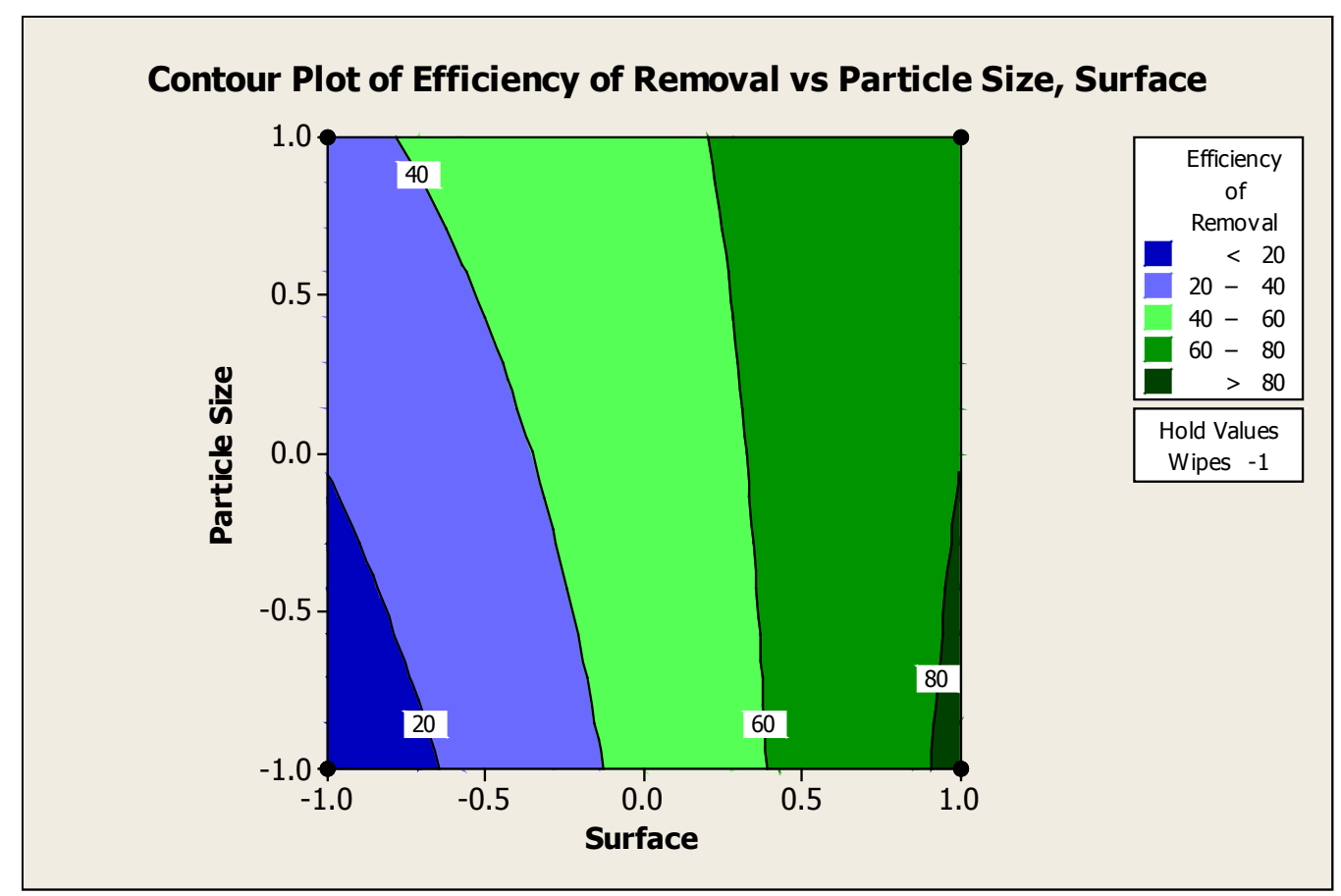

Figure 46 Contour plot of the efficiency of loose contamination removal, particle size of the contaminant(y axis) versus roughness of the surfaces (x axis) for wipe 2 


\subsubsection{Surface energy \& particle size of the contaminant influence on the efficiency of loose contamination removal: Model population \& data processing}

In order to evaluate the influence of the factors predicted by the JKR theory on the efficiency of loose contamination removal, a second experimental model was designed in which the surface energy will vary in two levels: hydrophobic (high level, in a PVC surface) and hydrophilic (low level, in a silica surface) (section 4.5.9). With that purpose, a second order of technetium pertecnetate with an activity of $5 \mathrm{mCi}$ in $5 \mathrm{ml}$ was received. Two samples, $1 \mathrm{ml}$ each, were extracted from the vial containing the isotope (Table 25). Then, as explained in section 4.5.4, the efficiency of the labeling of ${ }^{99 \mathrm{~m}} \mathrm{Tc}$ to the Dowex $50 \mathrm{Wx} 2$ resin was determined. The numbers presented in Table 26 are the activity in the supernatant and the resin.

Table 25 Initial activity used for labeling the particles in a 200 and 400 mesh

\begin{tabular}{|c|c|c|}
\hline Volume (ml) & Size (mesh) & Activity (mCi) \\
\hline 1 & 200 & 1.49 \\
\hline 1 & 400 & 1.49 \\
\hline
\end{tabular}

Table 26 Activities measured in the supernatant and the solid after labeling

\begin{tabular}{|l|c|c|}
\hline & 200 Mesh Activity (mCi) & 400 Mesh Activity (mCi) \\
\hline Initial & 1.49 & 1.49 \\
\hline Supernatant & 0.35 & 0.25 \\
\hline Resin & 1.03 & 1.21 \\
\hline
\end{tabular}

The values reported in Table 26 were used to calculate the efficiency of the labeling process, which is the amount of radioactive Tc-99m bonded to the resin:

$$
\eta_{200}=\frac{A_{i 200}-A_{s 200}}{A_{i 200}}=\frac{1.49-0.35}{1.49}=0.820 \stackrel{\text { yields }}{\longrightarrow} 82.0 \%
$$




$$
\eta_{400}=\frac{A_{i 400}-A_{s 400}}{A_{i 400}}=\frac{1.49-0.25}{1.49}=0.832 \stackrel{\text { yields }}{\longrightarrow} 83.2 \%
$$

Where $A_{i 200}$ and $A_{i 400}$ (in milicuries) were the initial activities of Tc-99m in the solutions with particles of mesh size 200 and 400, respectively; and $A_{s 200}$ and $A_{s 400}$ were the activities of Tc-99m in the supernatant with particles of mesh size 200 and 400, respectively.

In Table 27, the data collected from the experiment is summarized.

Table 27 Populated matrix with the experimental values obtained, following the order presented in Table 2 for surface energy considerations

\begin{tabular}{|l|l|l|l|l|l|l|l|l|}
\hline Order & No. & A & B & C & Before & After & Time in & Time out \\
\hline 4 & 1 & W2 & GLASS & 200 & 21.5 & 6.82 & $11: 06$ & $11: 08$ \\
\hline 17 & 2 & W1 & GLASS & 200 & 23.4 & 7.82 & $12: 00$ & $12: 02$ \\
\hline 11 & 3 & W2 & PVC & 200 & 19.1 & 5.95 & $11: 35$ & $11: 37$ \\
\hline 20 & 4 & W1 & PVC & 200 & 17.26 & 5.27 & $12: 12$ & $12: 14$ \\
\hline 7 & 5 & W2 & GLASS & 400 & 24.2 & 5.66 & $11: 19$ & $11: 21$ \\
\hline 13 & 6 & W1 & GLASS & 400 & 22.3 & 5.05 & $11: 43$ & $11: 45$ \\
\hline 3 & 7 & W2 & PVC & 400 & 22.6 & 3.29 & $11: 01$ & $11: 03$ \\
\hline 21 & 8 & W1 & PVC & 400 & 19.16 & 2.29 & $12: 19$ & $12: 21$ \\
\hline 1 & 9 & W2 & GLASS & 200 & 24 & 7.62 & $10: 52$ & $10: 54$ \\
\hline 5 & 10 & W1 & GLASS & 200 & 22.3 & 7.21 & $11: 10$ & $11: 12$ \\
\hline 14 & 11 & W2 & PVC & 200 & 18.74 & 4.99 & $11: 47$ & $11: 49$ \\
\hline 18 & 12 & W1 & PVC & 200 & 17.74 & 5.64 & $12: 04$ & $12: 06$ \\
\hline 16 & 13 & W2 & GLASS & 400 & 21.4 & 4.52 & $11: 55$ & $11: 58$ \\
\hline 10 & 14 & W1 & GLASS & 400 & 23.2 & 5.53 & $11: 31$ & $11: 33$ \\
\hline 9 & 15 & W2 & PVC & 400 & 22.5 & 3.01 & $11: 27$ & $11: 29$ \\
\hline 12 & 16 & W1 & PVC & 400 & 22.2 & 4.08 & $11: 39$ & $11: 41$ \\
\hline 19 & 17 & W2 & GLASS & 200 & 20.3 & 8.56 & $12: 08$ & $12: 10$ \\
\hline 22 & 18 & W1 & GLASS & 200 & 21.6 & 8.39 & $12: 22$ & $12: 24$ \\
\hline 23 & 19 & W2 & PVC & 200 & 13.86 & 1.37 & $12: 26$ & $12: 28$ \\
\hline 24 & 20 & W1 & PVC & 200 & 15.9 & 5 & $12: 30$ & $12: 32$ \\
\hline 8 & 21 & W2 & GLASS & 400 & 23.9 & 5.41 & $11: 23$ & $11: 25$ \\
\hline 15 & 22 & W1 & GLASS & 400 & 23.5 & 5.2 & $11: 51$ & $11: 53$ \\
\hline 6 & 23 & W2 & PVC & 400 & 21.8 & 1.86 & $11: 14$ & $11: 16$ \\
\hline 2 & 24 & W1 & PVC & 400 & 21.2 & 3.8 & $10: 56$ & $10: 59$ \\
\hline & & & & & & & & \\
\hline
\end{tabular}


After that, the surfaces were contaminated (as described in section 4.5.10) and the amount of contamination removed was determined by measuring the contamination remaining on the surfaces (before and after wiping) and the amount of contamination entrapped on the wipes, respectively.

The values presented in Table 27 were corrected by time (see Appendix 9.4) and the values of efficiency of loose contamination were found (Table 28).

Table 28 Values of efficiency of removal of loose contamination for surface energy considerations

\begin{tabular}{|c|c|c|c|}
\hline Order & Surface & Factorial & Efficiency of contamination removal (\%) \\
\hline 4 & 1 & 1 & 68.67 \\
\hline 17 & 2 & $\mathrm{a}$ & 66.91 \\
\hline 11 & 3 & $\mathrm{~b}$ & 69.31 \\
\hline 20 & 4 & $\mathrm{ab}$ & 70.01 \\
\hline 7 & 5 & $\mathrm{c}$ & 77.03 \\
\hline 13 & 6 & $\mathrm{ac}$ & 77.03 \\
\hline 3 & 7 & $\mathrm{bc}$ & 86.00 \\
\hline 21 & 8 & $\mathrm{abc}$ & 88.75 \\
\hline 1 & 9 & 1 & 68.59 \\
\hline 5 & 10 & $\mathrm{a}$ & 68.04 \\
\hline 14 & 11 & $\mathrm{~b}$ & 73.91 \\
\hline 18 & 12 & $\mathrm{ab}$ & 68.71 \\
\hline 16 & 13 & $\mathrm{c}$ & 79.36 \\
\hline 10 & 14 & $\mathrm{ac}$ & 76.60 \\
\hline 9 & 15 & $\mathrm{bc}$ & 87.20 \\
\hline 12 & 16 & $\mathrm{abc}$ & 82.15 \\
\hline 19 & 17 & 1 & 58.13 \\
\hline 22 & 18 & $\mathrm{a}$ & 61.47 \\
\hline 23 & 19 & $\mathrm{~b}$ & 91.14 \\
\hline 24 & 20 & $\mathrm{ab}$ & 69.14 \\
\hline 8 & 21 & $\mathrm{c}$ & 77.80 \\
\hline 15 & 22 & $\mathrm{ac}$ & 78.41 \\
\hline 6 & 23 & $\mathrm{bc}$ & 92.12 \\
\hline 2 & 24 & $\mathrm{abc}$ & 82.60 \\
\hline & & & \\
\hline
\end{tabular}


In the third replication of the experiment, a high variability between observations of the same type was found. Two outlier values severely depart from the trend of observations presented in replications 1 and 2 (see surface number 19 and 23, in Table 27). It was decided to neglect the third replication and analyze the first and second replication only (16 observations). Table 28 summarized the values of efficiency of removal of loose contamination removed from the surfaces. Note again that surfaces 19 and 23 depart from the regular observations trend.

\subsubsection{Surface energy data analysis}

The data was analyzed as explained in previous sections; Table 29 summarizes the effect of each factor in the model and their interaction. In addition, it can be observed (Table 29) that the significant factors within the model are the particle size of the contaminant, the surface energy, and their mutual interaction, as predicted by the JKR theory. The ANOVA table confirms the linearity of the model.

Table 29 Estimated effects and coefficients for observations presented in Table 20

\begin{tabular}{|l|c|c|c|c|c|}
\hline Term & Effect & Coef & SE & T & P \\
\hline Constant & & 75.52 & 0.54 & 139.87 & $<0.001$ \\
\hline Wipes & -1.483 & -0.74 & 0.54 & -1.37 & 0.207 \\
\hline Surface Energy & 5.474 & 2.73 & 0.54 & 5.07 & 0.001 \\
\hline Particle size & 12.497 & 6.24 & 0.54 & 11.57 & $<0.001$ \\
\hline Wipes*Surface Energy & -0.217 & -0.11 & 0.54 & -0.20 & 0.845 \\
\hline Wipes*Particle size & 0.220 & 0.11 & 0.54 & 0.20 & 0.843 \\
\hline Surface Energy*Particle size & 3.042 & 1.52 & 0.54 & 2.82 & 0.023 \\
\hline Wipes*Surface & 0.331 & 0.17 & 0.54 & 0.31 & 0.767 \\
\hline
\end{tabular}


Table 30 Summary of ANOVA table for surface energy considerations

\begin{tabular}{|l|c|c|c|c|l|l|}
\hline Source & DF & Seq SS & Adj SS & MS & F & P \\
\hline Main Effects & 3 & 753.396 & 753.396 & 251.132 & 53.84 & $<0.001$ \\
\hline 2-Way Interactions & 3 & 37.402 & 37.402 & 12.467 & 2.67 & 0.118 \\
\hline 3-Way Interactions & 1 & 0.438 & 0.438 & 0.438 & 0.09 & 0.767 \\
\hline Residual Error & 8 & 37.316 & 37.316 & 4.665 & & \\
\hline Pure Error & 8 & 37.316 & 37.316 & 4.665 & & \\
\hline Total & 15 & 828.553 & & & & \\
\hline
\end{tabular}

From Table 29, the column presenting the coefficients can be rewritten as:

$$
\mathrm{y}=75.53-0.47 \mathrm{x}_{1}+2.73 \mathrm{x}_{2}+6.24 \mathrm{x}_{3}-0.10 \mathrm{x}_{1} \mathrm{x}_{2}+0.11 \mathrm{x}_{1} \mathrm{x}_{3}-1.52 \mathrm{x}_{2} \mathrm{x}_{3}+0.16 \mathrm{x}_{1} \mathrm{x}_{2} \mathrm{x}_{3}
$$

Where $\mathrm{y}$ is the efficiency of loose contamination removal $(\%)$ and $\mathrm{x}_{1}, \mathrm{x}_{2}$, and $\mathrm{x}_{3}$ are the coded variables that represent the wipe used, the surface energy and the particle size of the contaminant, respectively.

The empirical equation presented above can be modeled in a $2 \mathrm{D}$ graph. A $2 \mathrm{D}$ representation of equation 33 is presented in Figure 47. Figure 47 represents the prediction values of efficiency of loose contamination removal when cleaning both wipes, and varies the particle size of the contaminant between $37-74 \mu \mathrm{m}$ and $74-149 \mu \mathrm{m}$ ( 1 and -1 , respectively) and the surface energy of the surface ( 1 and -1 are surfaces hydrophobic, PVC, and -1 means surfaces hydrophilic, silica) in which the contamination is located.

Table 31 Regression fit estimators of the empirical model

\begin{tabular}{|l|l|}
\hline $\mathrm{S}$ & 2.2 \\
\hline $\mathrm{R}-\mathrm{Sq}$ & $95.5 \%$ \\
\hline PRESS & 149.3 \\
\hline R-Sq(pred) & $81.9 \%$ \\
\hline R-Sq(adj) & $91.6 \%$ \\
\hline
\end{tabular}


In Appendix 9.5.1 is presented the graphs of normality plot of the residuals and the residuals versus fitted value, particle size of the contaminant and surface roughness, respectively.

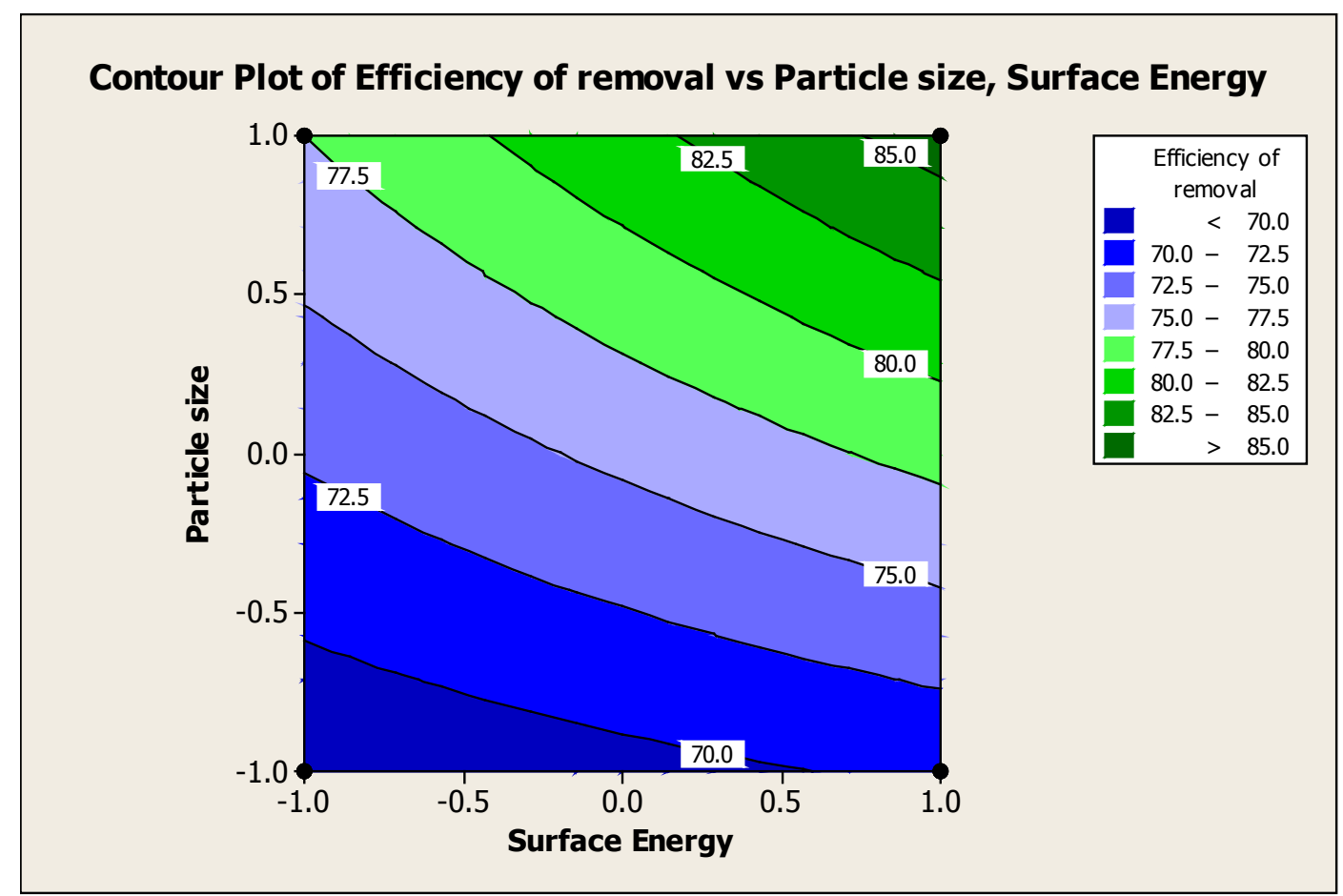

Figure 47 Contour plot of the efficiency of loose contamination removal, particle size of the contaminant(y axis) versus surface energy ( $\mathrm{x}$ axis) for both wipes

The particle size of the contaminant and variations of the surface energy of the surface are the main factor that affected the amount of contamination removed (P-value table 29, and figure 47). It can also be observed that when the particle size increased, decreased the amount of contamination removed. The detachment force predicted by the JKR theory depends upon the particle size of the contaminant and the work of adhesion between the contaminant and the surface. The resin is composed of water (50\% or more) then adhered with greater strength on glass surfaces than of PVC surfaces (Figure 47 
variations in the horizontal axis changed the amount of contamination removed, -1 is glass, 1 PVC). As a result, the work of adhesion increased and the amount of contamination removed is less. 


\subsection{DISCUSSION}

Loose contamination is present everywhere. Dealing with scenarios in which loose contaminants are presented is a very complicated process because exposure to loose contaminants can cause internal deposition of hazardous chemicals or unstable isotopes to workers exposed directly or indirectly to the contaminated areas. Therefore, predicting the amount of contamination removed beforehand can help reduce the exposure to loose contaminats.

Mathematical theories developed so far explain the detachment force necessary to remove a particle from a surface [21] but those theories neither account for the amount of contamination removed when cleaning a surface by wiping procedures nor account for the influence of the roughness of the surface in the process of decontamination of surfaces. However, experimental results obtained by Klein et al. [6] and Campbell et al. [26] have demonstrated that the physical properties of the contaminant, the surface and the wipe used, affected the amount of contamination removed, but neither of the mentioned studies accounted for the influence of the particle size of the contaminant in the process of removal of contamination. Therefore, it is necessary to evaluate the influence of the particle size of the contaminant in the process of contamination removal. As a result, the interactions that directly affects the amount of contamination removed from surfaces by wiping procedures need to be studied. Those interactions are based on the variation of the factors explained by the JKR theory that affect the strength of the detachment force necessary to remove a particle of contaminant from a surface (particle size of the contaminant and likeliness contaminant-surface), the roughness of the surface, 
and the wipe device used to remove the contamination. The objective of this research was to analyze the influence of the interaction between a loose contaminant and the surface it is on; and the influence of the interaction between the loose contaminant and the wipe device used to remove the contamination on the amount of contamination removed. This study will help to assess the value of contamination removal based on similar scenarios as the one modeled on this research.

Three scenarios were modeled to study the influence of the mentioned interactions. The first scenario consisted in modeling a contamination by a fluorescent powder of particle size of $5 \mu \mathrm{m}$ average on surfaces with different wetting behavior (hydrophobic or hydrophilic), and removing the contamination with wipes with different physical properties (absorbent and hydrophobic wipe). The second scenario was created by using a uranium oxide contaminant (like the one presented at nuclear power plants) in a particle size of $38 \mu \mathrm{m}$ on surfaces with different wetting behavior and different level of roughness. The uranium oxide was decontaminated using two hydrophobic wipes and a third wipe with a surfactant adhered to its microfibers. Finally, the third scenario was modeled by creating a $2^{3}$ factorial model. Three factors (particle size of the contaminant, surface roughness and wipe device used) were modeled at two levels (high and low) to evaluate the combining effect of the particle size of the contaminant, surface roughness and wipe used on the amount of contamination removed. Additionally, the change of wetting behavior of the surface was also analyzed. The contaminant used was a Dowex $50 \mathrm{~W} \times 2$ organic resin labeled with ${ }^{99 \mathrm{~m}} \mathrm{Tc}$ in two ranges of particle size, $37-74 \mu \mathrm{m}$, and $74-$ $149 \mu \mathrm{m}$. 
Initially, the characterization of the wipes used to remove contamination was conducted. Four wipes were studied, two wipes with a marked hydrophobic behavior, a third wipe with an absorbent behavior, and the last with a dual behavior (surfactant on its microfibers). The influence of the interaction of loose contaminant-wipes was significant for particles of size in the order of 4-5 $\mu \mathrm{m}$ on average (P-value in tables 33 through 35 , appendix 9.1), when the wetting properties of the contaminant and the wipes matched the amount of contamination removed increased. However, for particles between 37-149 $\mu \mathrm{m}$, this effect was not appreciable and the amount of contamination removed by each wipe used was the same.

Additionally, the characterization of the contaminated surfaces was conducted prior to contamination. A Drop Master equipment was used to evaluate surface wetting behavior (hydrophobic or hydrophilic), and an Atomic Force Microscopy (AFM) was used to indicate the level of roughness of the surfaces.

The particles characterization was made for three different contaminants: a thermo-plastic dye, a uranium oxide powder and an organic resin. The three contaminants were completely different in size, form and composition. The thermo-plastic contaminant has a size of $5 \mu \mathrm{m}$ in average, and an irregular form, while the uranium oxide was grinded to a size of $38 \mu \mathrm{m}$. Finally, the resin was the only contaminant that was perfectly spherical (optical characterization), in a size of 37-74 $\mu \mathrm{m}$ and 74-149 $\mu \mathrm{m}$ each. In this research, the influence of a different shape of the contaminant was not evaluated. The interaction of physical properties of the contaminant with the wipe used and the surface in which was spread was evaluated. 


\subsection{First scenario: Quantification of the contamination by weighing procedures}

The results presented with this method related the prediction by the JKR theory with the amount of contamination removed (see section 5.2). A hydrophobic contaminant of particle size 4-5 $\mu \mathrm{m}$ average was spiked on surfaces with different wetting behavior; when the contaminant and the surface were of the same nature (thermoplastic pigmentPVC surface, hydrophobic-hydrophobic); the JKR theory predicts an increase in the detachment force due to an increase on the adhesion work [21]. This was reflected in a decrease of the total amount of contamination removed when compared with the interaction hydrophobic-hydrophilic (thermoplastic pigment-ceramic like tile surface). This result confirmed the predictions by the JKR theory, which is an increase of the detachment force when increases the van der Waals force, and consequently, the work of adhesion between the contaminant and the surface [2, 3, 21, and 26]. This means that when the contaminant and the surface had similar wetting behavior the detachment force is higher than when their wetting behaviors differs, for a fixed contaminant particle size. As a result, the amount of contamination removed was higher on surfaces when the wetting behavior of the contaminant and the surface was different In addition, the interaction wipe-contaminant played an important role; the wipe with the hydrophobic behavior removed more contamination (Y-12 wipe) than the wipe with the absorbent behavior (wipe 4 Scott Multifold). The results obtained agree with previous results, (Campbell et al. [26]); an increase of contamination removal was observed when the contaminant and the wipe had similar polarities (or wetting behaviors). The surfaces used were never treated to get any special level of smoothness; they were used as regular ordinary surfaces that needed to be cleaned with a dry wipe. 
In this scenario, the order of magnitude of the response of the system (weight) was in micrograms, which means that the signal could be very sensitive to relative humidity in air, dust particles, technician performing the wipes, gloves and the precision and adjustment of the measurement equipment. When compared the weight of the contaminant with the relative weight of the wipe was not high (the relative weight of the contaminant divided by the relative weight of the wipe per surface was of $8 \%$ and $11 \%$ for Y-12 wipe and Scott wipe, respectively). Even though, the variability per replications were $10 \%, 13 \%$ and $7 \%$ for $\mathrm{Y}-12$ wipe and $19 \%, 13 \%$ and $10 \%$ for Scott wipe for the surfaces of PVC, Formica, and ceramic, respectively. The $19 \%$ variability on the PVC surfaces with the Scott wipe (towels) was due to an outlier point (without this point the variability of the observations decreased to $13 \%$ ), besides, the Mean Square of the Error (MSE) presented by the ANOVA method was not high enough in comparison with Mean Square of Wipes (appendix 9.1 table 1), this meant a high F-value able to reject the null hypothesis and conclude that the difference per wipe used was significant. That said, these results indicate a good reproducibility of the results and overall performance of the experiment.

\subsection{Second scenario: Quantification of the contamination by detection of alpha particles}

In order to improve the quantification of the contamination from the previous method, an experiment was designed in which the contamination was alpha particles coming from ${ }^{238} \mathrm{U}$ present in uranium oxide $\left(\mathrm{U}_{3} \mathrm{O}_{8}\right)$ in a particle size of $38 \mu \mathrm{m}$. The results are summarized in Appendix 9.2 and in Figures 40 and 41, section 5. The results obtained by wiping procedures were in correlation with the strength of the detachment force predicted 
by the JKR theory [21]. For surfaces of PVC (hydrophobic), more contamination was removed than for surfaces of ceramic or Formica (hydrophilic); their difference in wetting behavior was the cause of the observations. These difference indicates an increase of the van der Waals forces when their physical properties were similar [3, 26] (same wetting behavior, uranium powder on Tile surface, hydrophilic-hydrophilic interaction), then a decrease in the amount of contamination removed was observed when increased the interaction contaminant-surface, and vice versa. Previous results (Campbell et al [26]) also demonstrated that the affinity between the contaminant and the surface influences the amount of contamination removed. The wipe-contaminant interaction behaves similarly when using either the hydrophobic wipes (Y-12 wipes) or the wipe with the surfactant adhered to its microfibers (Chicopee Maslin, wipe 3). The roughness of the surface decreases the amount of contamination removed for particles of $38 \mu \mathrm{m}$ (concrete and stainless steel) independently of the wipe device used to remove the contamination. However, when the contamination was removed with Chicopee-Maslin wipe the amount of contamination removed was higher than when removed with the other wipes (Y-12 wipes), this maybe because of the surfactant adhered to the microfiber of the Chicopee wipes that could reach deeper into the porous of the concrete surfaces, hence removing more contamination.

However, a disadvantage of this quantification method was that ${ }^{238} \mathrm{U}$ had a long half-life, so a long time of counting was necessary to quantify the contamination. Still, from the physical point of view, alpha particles have self absorption; if two layers of powder are one on top of the other, the photon of the lower layer will not be counted by the detector 
[15]. In our experiment, the self absorption phenomenon was hard to control due to the

small particle size of the uranium oxide. Also, when the ${ }^{238} \mathrm{U}$ was trapped within the microfiber, the alpha particle couldn't reach the detector, increasing the variability per surface and overall performance of the experiment.

The amount of uranium spiked per surface never exceeded $10 \mathrm{mg}$; this is a small amount and probably any wipe could remove this contamination to lower than the levels of detection without difficulty.

\subsection{Third Scenario: Quantification of the contamination by detection of the gamma photons}

Two experimental models were designed to test the hypothesis and objective of the research. The first model tested the influence of the roughness, the particle size of the contaminant and the wipe used. The model identified that the particle size of the contaminant, the surface roughness and their mutual interaction affected significantly the amount of contamination removed. There was not a difference in the wipe used for particles in the range of $37 \mu \mathrm{m}-149 \mu \mathrm{m}$; both removed the same amount of contamination when used on PVC or concrete within the range of particle size evaluated. The model also predicted the amount of contamination removed for particles sizes between $37 \mu \mathrm{m}$ $149 \mu \mathrm{m}$, and for surfaces of roughness in between PVC and concrete. In real life, resins are used to separate hazardous chemical or radioactive elements from a solution, then a process of packing and decommissioning is used to properly dispose of the resin. The risk of contamination of surfaces during this process is possible, so this scenario can help to 
predict the values of contamination removed when entering into contaminated areas with resins and surfaces of similar compositions to the ones modeled.

The second experimental model tested the influence of the amount of contamination removed when the surface energy of the surface and the particle size of contaminant were varied. The values obtained reflected that, when the surface and the contaminant had similar wetting behavior, the van der Waal forces between the contaminant and the surface increased [2,3, and 21], consequently, the work of adhesion increased [3], and as a result, the amount of contamination removed decreased (Campbell et al.[26]). Also, the particle size played a critical role in the results obtained. When the particle size of the contaminant became smaller $(37-74 \mu \mathrm{m})$, the amount of contamination removed was greater than when the particle size was bigger $(74-149 \mu \mathrm{m})$. These agree with the predictions of the JKR theory that the detachment force increased with the particle radii [3, 21], and the results obtained by Klein et al.[6], and Campbell et al.[26]; consequently the interaction contaminant-surface increased, and as a result, the amount of contamination removed from the surface decreased.

\subsection{Summary}

The empirical models developed in sections 5.4.4 and 5.4.7 were not intended to extrapolate the values of contamination removal to a particle size greater or smaller than the ones presented $(37-149 \mu \mathrm{m})$ or to surfaces different from the one tested, or different wipes characteristics. The purpose was to analyze how variations of the particle size of the contaminant, the surface energy of the surface, and the surface roughness affected the interaction contaminant-surface and the interaction contaminant-wipe by quantifying the 
amount of contamination removed. Also evaluated were if those variations affected in the same ratio the detachment force explained in the JKR theory. Generally, empirical models are useful tools for determination of local interpolation [20]. The regression equations of the empirical models obtained explained the variability of the observations with a confident greater than $90 \%$ and predict an interpolated point with a precision greater than $90 \%$ also.

The interaction contaminant-wipe only played a significant role when used particles on 5 $\mu \mathrm{m}$ average size, but not when the particles increased from $37-149 \mu \mathrm{m}$. A possible reason for this effect was the relation of particle size of the contaminant and width of the microfiber of the wipes (Figures 48 and 49). When the particle size of the contaminant was smaller than the width of the microfiber (Figure 49), the interaction contaminantwipe was significant. This meant that when the contaminant and the wipe had similar wetting behavior, the van der Waal forces increased, and more contamination was removed from the surface than when a wipe with absorbent behavior was used (Campbell et al. [26]). The influence of the van der Waal forces depends on the particle size of the contaminant (Quesnel et al. [3]), for contaminants of size in between 37-149 $\mu \mathrm{m}$ the effect of these forces was not appreciable. Then, the amount of contamination removed was independently of the wipe used, but dependent on the interaction between the contaminant and the surface. 


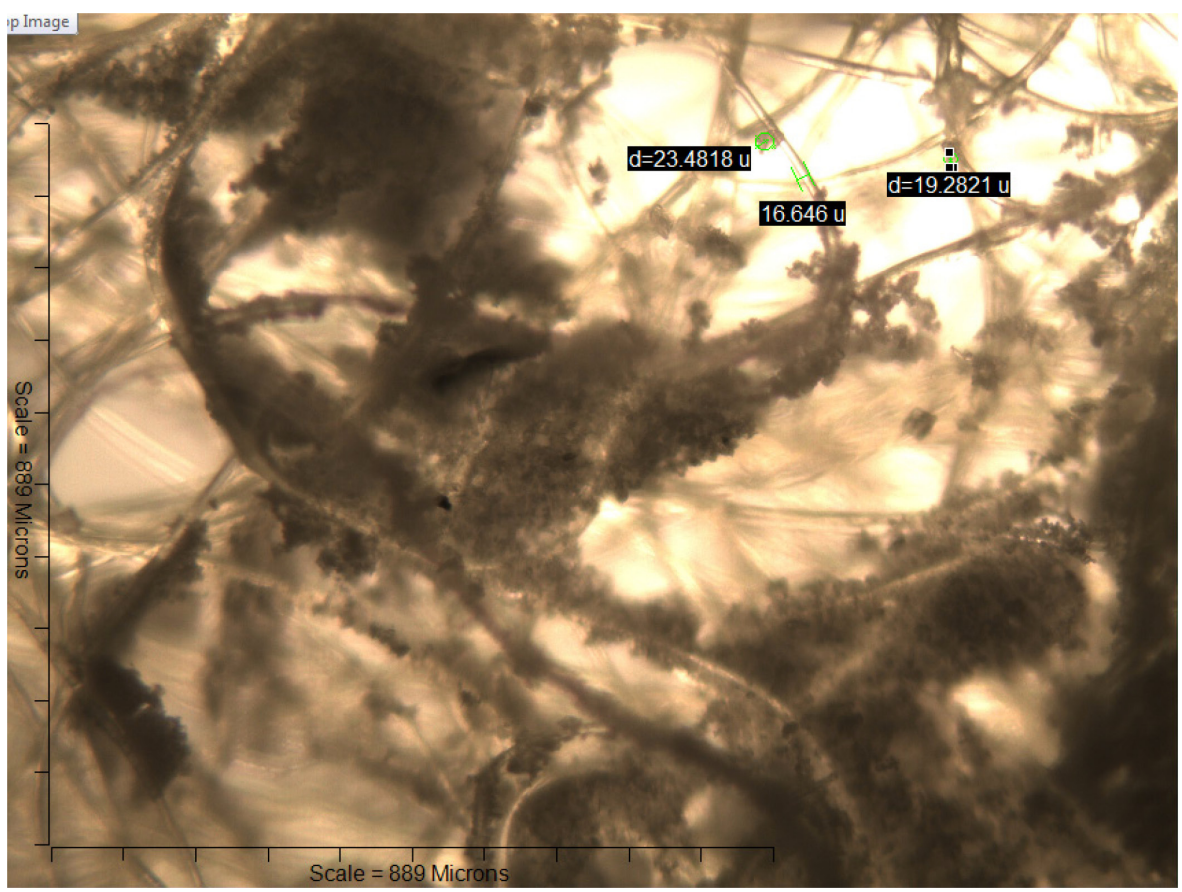

Figure 48 Thermo-plastic on the Chicopee-Maslin microfibers viewed at $100 \mathrm{x}$ magnification ( 889 microns is the total length of the scale)

In this research, the amount of contamination removed was predicted based on the interaction contaminant-surface (work of adhesion between contaminant-surface), the interaction wipe-contaminant, and roughness of the surface. However, there are additional factors reported in the literature that might affect the amount of contamination removed [3, 8]. Those factors include the roughness of the contaminant and the environmental conditions in which the contaminant and the surface are exposed (such as relative humidity, temperature, etc [8]). During the performance of the experiment, those factors were kept constant and the experiments were carried out under controlled conditions (inside a fume hood with constant ventilation, and temperature, in a clean environment). The contributions of these factors were assumed to be under the error of the experiments performed in section 5 to quantify the contamination removed. 


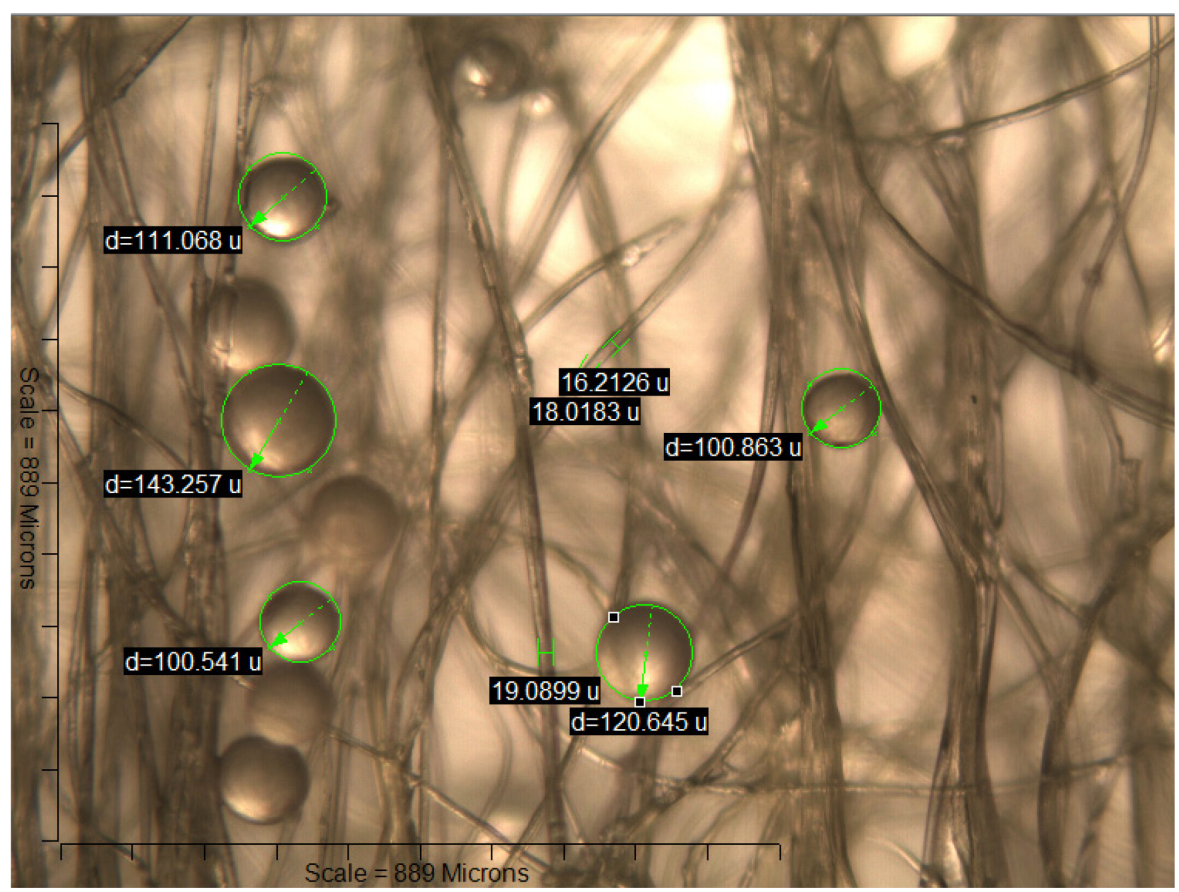

Figure 49 Dowex 50Wx2 resin on the Chicopee-Maslin microfibers viewed at $100 \mathrm{x}$ magnification ( 889 microns is the total length of the scale) 


\subsection{CONCLUSIONS AND FUTURE WORK}

The methods developed above had the objective of studying the dependence of the amount of contamination removed with the contaminant-surface and contaminant-wipe interaction. The following conclusions were reached from the results obtained

- The interaction contaminant-wipe had a significant effect for particles of $5 \mu \mathrm{m}$; when their polarities matched, the amount of contamination removed increased more than when they matched (Figure 39, section 5.2). However, for particles in the range of $37 \mu \mathrm{m}-142 \mu \mathrm{m}$, this interaction was not significant; the amount of contamination removed depended on the interaction contaminant-surface.

- The interaction contaminant-surface had a significant effect for particles between 5 and $140 \mu \mathrm{m}$. Variations in the amount of contamination removed were observed when the physical properties between contaminants-surfaces changed (hydrophobic contaminant on a hydrophilic surface or otherwise), even when the surfaces were not perfectly smooth and the contaminant was not perfectly spherical (thermoplastic pigment and uranium oxide powder). Variations on the surface energy and particle size of the contaminant were observed that affected the amount of contamination removed, in the inverse proportion of the detachment force predicted by the JKR theory. This meant that when the detachment force decreased, the amount of contamination removed increased, and vice versa.

- The amount of contamination removed decreased when the roughness of the surface increased for particles from 37 to $142 \mu \mathrm{m}$. 
The conclusions presented above can help workers directly exposed in the field to efficiently remove contamination from surfaces. Based on the characteristics of the contaminant and the surface, the worker can choose the best available technology to efficiently remove the contamination from the surfaces.

The results from this research were already applied onsite. Contamination was discovered in a Rad-Lab (licensed to work with long half-life isotopes) in a weekly survey by direct surveying. The contaminated area was identified and the hot spots marked (Figure 50).

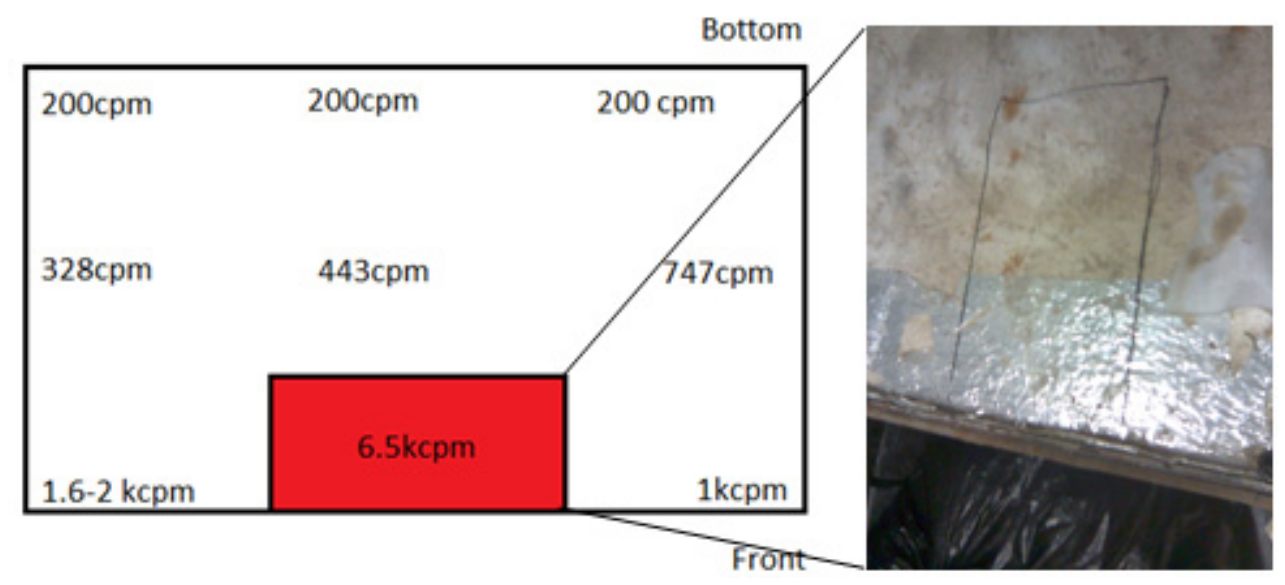

Figure 50 Hot spot area identification

The contamination discovered was unknown, but it was observed that the surface was plastic and that it was very rough. The contamination was mainly alpha and beta, a NaI scintillation detector was used to dismiss gamma radiation. The empirical models developed in sections 5.4.4 and 5.4.5 predicted that the amount of contamination removed on rough surfaces is small. Thus, it was decided to test the two types of wipe 
available for decontamination, in this case wipe 1 and wipe 2 . After wiping, the amount of contamination removed was 5-6\% for both wipes.

Table 32 Contamination detected during weekly survey at a Rad-Lab

\begin{tabular}{|c|c|c|c|}
\hline \multicolumn{4}{|c|}{ Evaluation of Beta contamination } \\
\hline $\begin{array}{c}\text { Order } \\
\text { No. }\end{array}$ & $\begin{array}{l}\text { Initial Contamination } \\
(\mathrm{kcpm})\end{array}$ & $\begin{array}{c}\text { Contamination remained after } \\
\text { wiping (kcpm) }\end{array}$ & $\begin{array}{l}\text { Efficiency of } \\
\text { removal (\%) }\end{array}$ \\
\hline 1 & 11.78 & 11.24 & 4.58 \\
\hline 3 & 10.89 & 10.68 & 1.93 \\
\hline & & $\begin{array}{l}\text { Contamination picked by the } \mathrm{Y}-12 \\
\text { wipe (cpm) }\end{array}$ & \\
\hline 1 & 11.78 & 784 & 6.66 \\
\hline 3 & 10.89 & 585 & 5.37 \\
\hline & $\begin{array}{l}\text { Initial Contamination } \\
(\mathrm{kcpm})\end{array}$ & $\begin{array}{c}\text { Contamination remained after } \\
\text { wiping }(\mathrm{kcpm})\end{array}$ & $\begin{array}{l}\text { Efficiency of } \\
\text { removal (\%) }\end{array}$ \\
\hline 2 & 11.22 & 10.89 & 2.94 \\
\hline & & $\begin{array}{l}\text { Contamination picked by the } \\
\text { Chicopee wipe(cpm) }\end{array}$ & \\
\hline 2 & 11.22 & 574 & 5.12 \\
\hline
\end{tabular}

The wiping process was performed three times and the contamination persisted. Wiping the contaminated area was not a feasible way to decontaminate the surface. The area was covered with a plastic paper to shield the beta and the alpha particles (Figure 51). Further decontamination methods need to be applied to this area (like decommission) in order to remove the contamination. This example shows a direct application of the results obtained from this experiment.

It is recommended in future work to evaluate the amount of contamination removed for particles of $5 \mu \mathrm{m}$ on rough surfaces. Also, it was demonstrated that empirical models fit 
the data, but sometimes the development of a mechanistic model is more helpful for extrapolation purposes and validation of the results [22], although testing an extrapolate value is a safe way to check the results from the developed model.

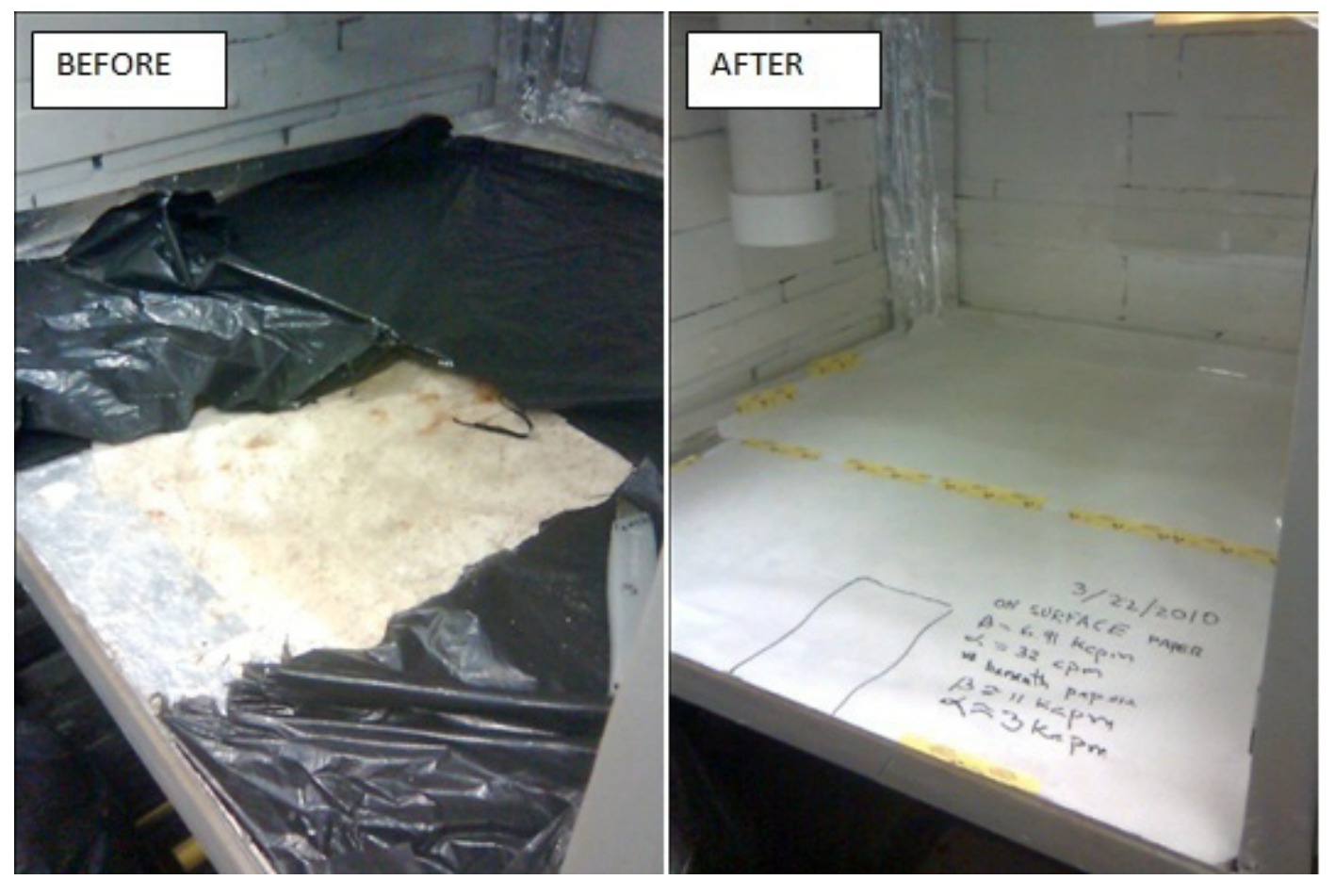

Figure 51 Area before and after decontamination efforts

Based on the conclusion of this study, the amount of contamination removed can be predicted based on the physical properties of the contaminant and the surface. However, in order to generalize the results obtained and a future successful application, the following is recommended:

1. Research the literature for the physical and chemical properties of the contaminant (including an estimation of the particle size).

2. Research the physical properties of the surfaces, including wetting behavior and roughness. 
3. Predict the strength of the contaminant-surface, and wipe-contaminant interaction.

4. Assess the impact of the environmental conditions that might be involved in the decontamination removal process, such as relative humidity, temperature, etc.

5. Develop a pilot experiment that reproduces as close as possible the real conditions and take advantage of the best scenario found. 


\section{BIBLIOGRAPHY}

1. Khilnani Arvind."Cleaning Semiconductors Surfaces : Facts and Foibles." In: Mittal K.L edts.. "Particles on Surfaces 1: Detection, Adhesion and Removal.’Plenun Press, NY: 17-35; 1988.

2. Myers Drew. "Surfaces, Interfaces and Colloids." Second edition, John Wiley and Sons Inc: 1-140; 1999.

3. Quesnel David J., Donals Rimai, David M Schaefer. "Aspects of Particle Adhesion and Removal." In: Kohly Rajiv, K.L Mittal, eds. "Developments in Surfaces Contamination and Cleaning: Fundamentals and Applied Aspects.’William Andrew :475-498; 2008.

4. U.S. Department of Health and Human Services. Public Health Service.Agency for Toxic Substance and Diseases Registry."Toxicological Profile for Beryllium". September 2002.

5. Lagos Leonel, Calderin Duriem, NGachin Merlin. "Removal of Loose contamination from Surfaces: Evaluation of SIMWyPES Technology”. Applied Research Center Florida international University (FIU) 2009.

6. Klein C. Robert, Iliona Linins, Edward L. Gershey. "Detecting Removable Surface Contamination". Health Physics Society 62(2):186-189; 1992

7. Klein C. Robert, Iliona Linins, Edward L. Gershey. "Removable Surface Contamination at a Biomedical Research Institution." Health Physics Society 72:296-299; 1997

8. Shoji Miki, Takashi Kondo, Akihiro Kijima, Yukinobu Shibao, Tomoko Nakajima, Koichi Yamada, and Nobuo Nemoto. "Removal Characteristics of Wipe Devices under Various Conditions." Health Physics Society 99:136-142; 2010

9. Kerr Kent "Sampling Beryllium Surface Contamination using wet, dry and alcohol wipe methods" Master Thesis, Department of Safety Sciences, Central Missouri State University. December 2004.

10. B. Le Guen, A. Roupioz, B. Rabu, A. Bouvy, J. F. Labouglie and Y. Garcier. "Control of the Risk of Exposure to Alpha Emitting Radionuclides in French Nuclear Power Plants: Example of Catteton.'Nuclear Protection Dosimetry. Vol 105: 303-309; 2003.

11. Montgomery C. Douglas. "Design and Analysis of Experiments." Sixth edition, John Wiley \& Sons, Inc :203-254; 2005. 
12. Burnham J. U. "Radiation Protection." New Brunswick Power Corporation, Rev. 4, 2001.

13. Redlich A. Carrie, Welch S. Laura. "Chronic Beryllium Disease: Risk from LowLevel Exposure." American Journal of Critical Care Medicine Vol. 177:936-937; 2008 .

14. U.S. Nuclear Regulatory Commission."Control of Radioactively Contaminated Materials." Washington DC: Office of Inspection and Enforcement. IE Circular No. 81-07; May 14, 1981.

15. Choppin R. Gregory, Liljenzin Jan-Olov, Rydberg Jan. "Radiochemistry and Nuclear Chemistry." Third Edition, Butterworth-Heinemann: 415-440; 2002.

16. Owunwanne Azu, Church B. Larry, Blau Monte. "Effect of oxygen on the Reduction of Pertechnetate by Stannous ion." Journal of Nuclear Medicine 18:822-826, 1977.

17. Dieck, R. "Measurement Uncertainty: Methods and Applications, Fourth Edition." Instrumentations, System, and Automation Society :9-147; 2007.

18. Chilton B. Arthur, j. Kenneth Shultis, Richard E. Faw. "Principles of Radiation Shielding." Prentice Hall, Inc :121-140; 1984.

19. U.S Nuclear Regulatory Commission. "Consolidate Guidedance about Materials Licenses." Washington DC: Office of Nuclear Materials Safety and Safeguards; NUREG-1556; Vol. 9; 2002.

20. Cember Herman. Johnson E. Thomas. "Introduction to Health Physics". McGraw Hill Companies Inc. Fourth edition:427-505; 2009.

21. Johnson K.L."Contact Mechanics.”Cambridge University Press: 84-104; 1985.

22. Box, P. E. Geoerge, William G. hunter, J.Stuart Hunter. "Statistics for experimenters: An Introduction to Design, Data Analysis and Model Building." John Wiley \& Sons :540-552, 1978.

23. Pashley M. Richard, Marilyn E. Camaran. "Applied Colloids and Surface Chemistry.” John \&Wiley Sons Ltd.: 13-42, 2004.

24. Hetch Lewis. "An Introductory Review of Particles Adhesion to Solid Surfaces." Journal of International Education Studies: 33-37, 1990.

25. Verdegan M. Barry, Dryan Schwadt. "Preferential Attachment of Contaminants to Filter Media in Oil.” Filtration \& Separation: 1061-1064, 1997. 
26. Campbell L Jerry, Charles R. Santerre."Wipe Testing for Surface Contamination by Tritiated Compounds." Journal of Health Physics, Operational topics; 64(5): 540-544, 1993.

27. Jung H, Kunze JF, NurrenbernJD. "Consistency and efficiency of standard swipe procedures taken on slightly radioactive contaminated metal surface." Health Physics Society 80:S80-88; 2001.

28. Wang Xiaolin, Yibei Fu. "Surface Chemical Behavior of Triuranium Octaoxide in the Atmospheres of Carbon Monoxide and Hydrogen." Journal of Nuclear Materials; Vol 257: 287-294; 1998.

29. Karangelos D.J, M.J. Anagnostakis. "Determination of Depleted Uranium in Environmental Samples by Gamma-spectroscopic Techniques." Journal of Environmental Radioactivity; 76 :295-310; 2004.

30. Ranade, M. B. "Adhesion and Removal of Fine Particles on Surfaces." Aerosol and Technology; 72:161-176; 1987.

31. Ninkovic M.M., J.J. Raicevic, F Adrovic. " Air Kerma Rate Constants for Gamma Emitters Used Most Often in Practice." Radiation Protection Dosimetry; 15:247-250; 2005.

32. Rogers L.N., J.Reed. "The Adhesion of Particles Undergoing an Elastic-plastic Impact with a Surface." Journal of Physics, Applied Physics; 17:677-689;1984.

33. Pettit A. William, Frank H. Delagado, George H. Pepper, Linda Blanton. "Characterization of Tin-technetium Colloid in Technetium-labeled Albumin Preparations." Journal of Nuclear Medicine; 19:387-392;1978.

34. Cho W.D., Man He Han, Mark Bronson, Yury Zundelevich."Processing of Uranium Powders in a Fuidized-bed Reactor." Journal of Nuclear Materials; 305:106-111;2002.

35. Stetzer Olaf, Maria Betti, Jacobus van Geel, Nicole Erdmann, Jens-Volker Kratz, Roladn Schenkel, Nrobert Trautmann. "Determination of ${ }^{235} U$ Content in Uranium Oxide Powder Particles by Fission Track Analysis." Nuclear Instruments and Methods in Physics Research, Elsevier; 525: 582-592; 2004.

36. Cole David, Lei Zhang. "Surfaces analysis Methods for Contamination Identification." In: In: Kohly Rajiv, K.L Mittal, eds. "Developments in Surfaces Contamination and Cleaning: Fundamentals and Applied Aspects." William Andrew:585-647;2008

37. Clark G. Philip, Thomas J. Wagener. "Removal of Particles by Chemical Cleaning." In: Kohly Rajiv, K.L Mittal, eds. "Developments in Surfaces 
Contamination and Cleaning: Fundamentals and Applied Aspects." William Andrew: 873-888; 2008

38. Free L. Michael. "The Use of Surfactants to Enhance Particle Removal from Surfaces." In: Kohly Rajiv, K.L Mittal, eds. "Developments in Surfaces Contamination and Cleaning: Fundamentals and Applied Aspects."William Andrew: 727-753; 2008

39. Chao-Hsin Lin. "Relevance of Particle Transport in Surface Deposition and Cleaning." In: Kohly Rajiv, K.L Mittal, eds. "Developments in Surfaces Contamination and Cleaning: Fundamentals and Applied Aspects."William Andrew: 267-293; 2008 


\section{APPENDICES}

9.1 Data Collection and Evaluation of the contamination removed by weighing procedures

Table 33 Data collected to obtain the amount of contamination removed by weighing procedures on PVC surfaces

\begin{tabular}{|c|c|c|c|c|c|c|c|}
\hline \multicolumn{8}{|c|}{ Fluorescent Powder Data Analysis } \\
\hline \multicolumn{8}{|c|}{ PVC } \\
\hline \multicolumn{4}{|c|}{ Wipe 1} & \multicolumn{4}{|c|}{ Normal Wipe } \\
\hline $\mathrm{W}$ & WOP $(\mu g)$ & WP $(\mu \mathrm{g})$ & $\mathrm{P}(\mu \mathrm{g})$ & WN & WOP $(\mu \mathrm{g})$ & $\mathrm{WP}(\mu \mathrm{g})$ & $\mathrm{P}(\mu \mathrm{g})$ \\
\hline 1 & 190800 & 210300 & 19500 & 1 & 111400 & 124400 & 13000 \\
\hline 2 & 210000 & 226000 & 16000 & 2 & 105300 & 116200 & 10900 \\
\hline 3 & 203900 & 219800 & 15900 & 3 & 96400 & 113400 & 17000 \\
\hline 4 & 240200 & 257000 & 16800 & 4 & 106900 & 118700 & 11800 \\
\hline 5 & 193500 & 207800 & 14300 & 5 & 109000 & 120300 & 11300 \\
\hline 6 & 172800 & 188700 & 15900 & 6 & 108600 & 122200 & 13600 \\
\hline 7 & 191300 & 206400 & 15100 & 7 & 107600 & 116400 & 8800 \\
\hline 8 & 219700 & 238600 & 18900 & 8 & 107800 & 117500 & 9700 \\
\hline 9 & 188400 & 203800 & 15400 & 9 & 97700 & 107900 & 10200 \\
\hline 10 & 184300 & 200000 & 15700 & 10 & 98900 & 110000 & 11100 \\
\hline & & STD & 1641.30 & & & STD & 2338.18 \\
\hline & & Mean & 16350 & & & Mean & 11740 \\
\hline \multicolumn{8}{|c|}{ ANOVA Table Summary Results } \\
\hline & ource & DF & SS & & MS & $\mathbf{F}$ & $\mathbf{P}$ \\
\hline & Vipes & 1 & 106260500 & & $1.06 \mathrm{E}+08$ & 26.04 & $<0.001$ \\
\hline & Error & 18 & 73449000 & & 4080500 & & \\
\hline & Total & 19 & 179709500 & & & & \\
\hline
\end{tabular}

WOP: wipe without powder

WP: wipe with powder

P: weight of the powder 
Table 34 Data collected to obtain the amount of contamination removed by weighing procedures on Formica surfaces.

\begin{tabular}{|c|c|c|c|c|c|c|c|c|}
\hline \multicolumn{9}{|c|}{ Fluorescent Powder Data Analysis } \\
\hline \multicolumn{9}{|c|}{ Formica } \\
\hline \multicolumn{4}{|c|}{ Wipe 1} & \multicolumn{5}{|c|}{ Normal Wipe } \\
\hline $\mathrm{WN}$ & WOP $(\mu g)$ & WP $(\mu g)$ & $\mathrm{P}(\mu \mathrm{g})$ & WN & \multicolumn{2}{|c|}{ WOP $(\mu \mathrm{g})$} & $\mathrm{WP}(\mu \mathrm{g})$ & $\mathrm{P}(\mu \mathrm{g})$ \\
\hline 1 & 183480 & 204290 & 20810 & 1 & \multicolumn{2}{|c|}{98850} & 108300 & 9450 \\
\hline 2 & 199000 & 212830 & 13830 & 2 & \multicolumn{2}{|c|}{100360} & 111590 & 11230 \\
\hline 3 & 195170 & 213010 & 17840 & 3 & \multicolumn{2}{|c|}{107860} & 120060 & 12200 \\
\hline 4 & 190250 & 203570 & 13320 & 4 & \multicolumn{2}{|c|}{101470} & 109600 & 8130 \\
\hline 5 & 211420 & 228250 & 16830 & 5 & \multicolumn{2}{|c|}{95580} & 105860 & 10280 \\
\hline 6 & 185910 & 203140 & 17230 & 6 & \multicolumn{2}{|c|}{81220} & 89750 & 8530 \\
\hline 7 & 197580 & 216150 & 18570 & 7 & \multicolumn{2}{|c|}{88240} & 96940 & 8700 \\
\hline 8 & 181780 & 200600 & 18820 & 8 & \multicolumn{2}{|c|}{108610} & 117640 & 9030 \\
\hline 9 & 181270 & 197120 & 15850 & 9 & \multicolumn{2}{|c|}{94220} & 104910 & 10690 \\
\hline 10 & 195720 & 212690 & 16970 & 10 & \multicolumn{2}{|c|}{106860} & 117000 & 10140 \\
\hline & & STDV & 2260.654 & & & & STDV & 1301.954 \\
\hline & & Mean & 17007 & & & & Mean & 9838 \\
\hline \multicolumn{9}{|c|}{ ANOVA Table Summary of the Results } \\
\hline \multicolumn{2}{|r|}{ Source } & DF & \begin{tabular}{|l|l} 
SS & \\
\end{tabular} & \multicolumn{2}{|c|}{ MS } & $\mathbf{F}$ & \multicolumn{2}{|l|}{$\mathbf{P}$} \\
\hline \multicolumn{2}{|r|}{ Wipes } & 1 & 256972805 & $2.57 \mathrm{~B}$ & -08 & 75.52 & & \\
\hline & Error & 18 & 61250770 & 3402 & 321 & & & \\
\hline & Total & 19 & 318223575 & & & & & \\
\hline
\end{tabular}


Table 35 Data collected to obtain the amount of contamination removed by weighing procedures on ceramic surfaces.

\begin{tabular}{|c|c|c|c|c|c|c|c|c|}
\hline \multicolumn{9}{|c|}{ Fluorescent Powder Data Analysis } \\
\hline \multicolumn{9}{|c|}{ Ceramic } \\
\hline \multicolumn{4}{|c|}{$\begin{array}{c}\text { Wipe } 1 \\
\end{array}$} & \multicolumn{5}{|c|}{ Normal Wipe } \\
\hline $\mathrm{W}$ & WOP $(\mu \mathrm{g})$ & $\mathrm{WP}(\mu \mathrm{g})$ & $\mathrm{P}(\mu \mathrm{g})$ & $\mathrm{WN}$ & $\mathrm{WOP}$ & $(\mu \mathrm{g})$ & $\mathrm{WP}(\mu \mathrm{g})$ & $\mathrm{P}(\mu \mathrm{g})$ \\
\hline 1 & 208620 & 226240 & 17620 & 1 & 901 & & 109130 & 18940 \\
\hline 2 & 175760 & 194180 & 18420 & 2 & 105 & & 120820 & 14920 \\
\hline 3 & 186500 & 206730 & 20230 & 3 & 897 & & 107100 & 17360 \\
\hline 4 & 172370 & 193000 & 20630 & 4 & 101 & & 115490 & 14380 \\
\hline 5 & 182300 & 200770 & 18470 & 5 & 996 & & 115650 & 16020 \\
\hline 6 & 186500 & 207610 & 21110 & 6 & 100 & & 120400 & 19970 \\
\hline 7 & 192390 & 214160 & 21770 & 7 & 102 & & 120540 & 17670 \\
\hline 8 & 177710 & 196790 & 19080 & 8 & 103 & & 121040 & 17220 \\
\hline 9 & 172560 & 193870 & 21310 & 9 & 957 & & 111690 & 15990 \\
\hline 10 & 174440 & 192670 & 18230 & 10 & 102 & & 118970 & 16140 \\
\hline & & STDV & 1491.91 & & & & STDV & 1729.77 \\
\hline & & Mean & 19687 & & & & Mean & 16861 \\
\hline \multicolumn{9}{|c|}{ ANOVA Table summary of the Results } \\
\hline & ource & \begin{tabular}{l|l} 
DF &
\end{tabular} & SS & & MS & $\mathbf{F}$ & & 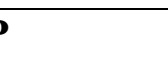 \\
\hline & Wipes & 1 & 39931380 & & 993138 & 15.31 & & $<0.001$ \\
\hline & Error & 18 & 46961300 & & 608961 & & & \\
\hline & Total & 19 & 86892680 & & & & & \\
\hline
\end{tabular}


9.2 Evaluation of the amount of contamination removed by alpha detection of U-238 present in uranium oxide (natural uranium)

Table 36 Table presenting the amount of uranium contamination removed from PVC surfaces

\begin{tabular}{|c|c|c|c|c|c|c|c|c|c|}
\hline & \multicolumn{6}{|c|}{$\begin{array}{l}\text { Radioactivity on Surface and Decontamination } \\
\text { Efficiency }\end{array}$} & \multicolumn{3}{|c|}{$\begin{array}{l}\text { Collection on Air Filter } \\
\text { Sample }\end{array}$} \\
\hline & $\begin{array}{r}\text { Initial l } \\
\text { Cour }\end{array}$ & $\begin{array}{l}\text { ioactiv } \\
\text { in } 2 \mathrm{Mi}\end{array}$ & $\begin{array}{l}\text { (Net } \\
\text { es) }\end{array}$ & & $\begin{array}{l}\text { ntamin } \\
\text { ciency }\end{array}$ & & & $\begin{array}{l}\text { dioac } \\
\text { ter/To } \\
\text { dioac } \\
\text { Surfa }\end{array}$ & $\begin{array}{l}\text { ty on } \\
\text { nitial } \\
\text { ty on } \\
\% \text { ) }\end{array}$ \\
\hline 1 & 2 & 3 & 4 & 5 & 6 & 7 & 8 & 9 & 10 \\
\hline Exp. & W3 & W1 & W2 & W3 & W1 & W2 & W3 & W1 & W2 \\
\hline 1 & 300 & 340 & 405 & 98.67 & 98.82 & 99.75 & 3.00 & 0 & 1.48 \\
\hline 2 & 497 & 452 & 499 & 99.20 & 97.12 & 99.40 & 0.80 & 0.22 & 0 \\
\hline 3 & 436 & 584 & 490 & 99.31 & 99.49 & 98.98 & 0.23 & 0.51 & 1.43 \\
\hline 4 & 508 & 363 & 354 & 93.50 & 97.52 & 96.89 & 0.20 & 3.31 & 1.98 \\
\hline 5 & 498 & 577 & 413 & 9.39 & 94.63 & 94.43 & 0.80 & 0.69 & 0.48 \\
\hline Mean & & & & 97.81 & 97.52 & 97.89 & 1.01 & 1.18 & 1.34 \\
\hline SD & & & & 2.44 & 1.88 & 2.23 & 1.15 & 1.43 & 0.63 \\
\hline RSU $^{*}$ & & & & $\mathbf{3 . 0 3}$ & 2.33 & 2.77 & & & \\
\hline
\end{tabular}


Table 37 Table presenting the amount of uranium contamination removed from Ceramic surfaces

\begin{tabular}{|c|c|c|c|c|c|c|c|c|c|}
\hline & \multicolumn{3}{|c|}{$\begin{array}{c}\text { Radioactivity on Surface and Decontamination } \\
\text { Efficiency }\end{array}$} & \multicolumn{3}{c|}{$\begin{array}{c}\text { Collection on Air Filter } \\
\text { Sample }\end{array}$} \\
\hline & $\begin{array}{c}\text { Initial Radioactivity } \\
\text { (Net Counts in 2 } \\
\text { Minutes) }\end{array}$ & \multicolumn{2}{c|}{$\begin{array}{c}\text { Decontamination } \\
\text { Efficiency (\%) }\end{array}$} & \multicolumn{2}{c|}{$\begin{array}{c}\text { Radioactivity on } \\
\text { Filter/Total Initial } \\
\text { Radioactivity on Surface } \\
\text { (\%) }\end{array}$} \\
\hline 1 & 2 & 3 & 4 & 5 & 6 & 7 & 8 & 9 & 10 \\
\hline Exp & W3 & W1 & W2 & W3 & W1 & W2 & W3 & W1 & W2 \\
\hline 1 & 443 & 509 & 485 & 97.74 & 90.57 & 92.37 & 0.68 & 1.57 & 1.44 \\
\hline 2 & 447 & 527 & 385 & 87.02 & 94.12 & 87.27 & 0.89 & 0.57 & 1.3 \\
\hline 3 & 494 & 397 & 484 & 91.30 & 90.68 & 89.05 & 1.42 & 0.50 & 0.83 \\
\hline 4 & 556 & 549 & 497 & 93.35 & 93.99 & 87.73 & 0.00 & 0.73 & 1.21 \\
\hline 5 & 520 & 570 & 560 & 86.92 & 89.82 & 88.75 & 0.58 & 1.23 & 0.54 \\
\hline Me & & & & $\mathbf{9 1 . 2 7}$ & $\mathbf{9 1 . 8 4}$ & $\mathbf{8 9 . 0 3}$ & $\mathbf{0 . 7 1}$ & $\mathbf{0 . 9 2}$ & $\mathbf{1 . 0 6}$ \\
\hline SD & & & & $\mathbf{4 . 5 6}$ & $\mathbf{2 . 0 5}$ & $\mathbf{2 . 0 0}$ & $\mathbf{0 . 5 1}$ & $\mathbf{0 . 4 6}$ & $\mathbf{0 . 3 7}$ \\
\hline RS & & & & $\mathbf{5 . 6 6}$ & $\mathbf{2 . 5 5}$ & $\mathbf{2 . 4 8}$ & & & \\
\hline
\end{tabular}


Table 38 Table presenting the amount of uranium contamination removed from Concrete surfaces

\begin{tabular}{|c|c|c|c|c|c|c|c|c|c|}
\hline & \multicolumn{6}{|c|}{$\begin{array}{l}\text { Radioactivity on Surface and Decontamination } \\
\text { Efficiency }\end{array}$} & \multicolumn{3}{|c|}{$\begin{array}{l}\text { Collection on Air } \\
\text { Filter Sample }\end{array}$} \\
\hline & \multicolumn{3}{|c|}{$\begin{array}{c}\text { Initial Radioactivity (Net } \\
\text { Counts in } 2 \text { Minutes) }\end{array}$} & \multicolumn{3}{|c|}{$\begin{array}{l}\text { Decontamination } \\
\text { Efficiency }(\%)\end{array}$} & \multicolumn{3}{|c|}{$\begin{array}{c}\text { Radioactivity on } \\
\text { Filter/Total Initial } \\
\text { Radioactivity on } \\
\text { Surface (\%) }\end{array}$} \\
\hline 1 & 2 & 3 & 4 & 5 & 6 & 7 & 8 & 99 & 10 \\
\hline Exp. & W3 & $\mathrm{W} 1$ & W2 & W3 & W1 & $\mathrm{W} 2$ & W3 & W1 & W2 \\
\hline 1 & 457 & 333 & 304 & 55.62 & 67.64 & 65.53 & 0 & 0.90 & 2.31 \\
\hline 2 & 401 & 550 & 334 & 58.90 & 75.50 & 74.23 & 2.50 & 1.82 & 0 \\
\hline 3 & 546 & 555 & 362 & 54.98 & 71.94 & 72.90 & 0.92 & 0.36 & 2.21 \\
\hline 4 & 426 & 500 & 475 & 60.14 & 66.64 & 62.50 & 1.64 & 1.40 & 1.26 \\
\hline 5 & 368 & 514 & 295 & 51.41 & 44.78 & 68.78 & 0.82 & 0.97 & 2.38 \\
\hline Mean & & & & 56.21 & 65.30 & 68.79 & 1.47 & 1.10 & 2.04 \\
\hline SD & & & & $\mathbf{3 . 4 5}$ & 12.01 & 4.92 & 0.78 & 0.55 & 0.52 \\
\hline RSU* & & & & 4.28 & 14.91 & 6.11 & & & \\
\hline
\end{tabular}


Table 39 Table presenting the amount of uranium contamination removed from Formica surfaces

\begin{tabular}{|c|c|c|c|c|c|c|c|c|c|}
\hline & \multicolumn{6}{|c|}{$\begin{array}{l}\text { Radioactivity on Surface and Decontamination } \\
\text { Efficiency }\end{array}$} & \multirow{2}{*}{\multicolumn{3}{|c|}{$\begin{array}{c}\text { Collection on Air } \\
\text { Filter Sample } \\
\text { Radioactivity on } \\
\text { Filter/Total Initial } \\
\text { Radioactivity on } \\
\text { Surface (\%) }\end{array}$}} \\
\hline & $\begin{array}{r}\text { Initial } \mathrm{P} \\
\text { Coun }\end{array}$ & $\begin{array}{l}\text { lioactiv } \\
\text { in } 2 \mathrm{Mi}\end{array}$ & $\begin{array}{l}\text { (Net } \\
\text { tes) }\end{array}$ & & $\begin{array}{l}\text { ontamir } \\
\text { iciency }\end{array}$ & & & & \\
\hline 1 & 2 & 3 & 4 & 5 & 6 & 7 & 8 & 9 & 10 \\
\hline Exp. & W3 & W1 & $\mathrm{W} 2$ & W3 & W1 & W2 & W3 & $\mathrm{W} 1$ & $\mathrm{~W} 2$ \\
\hline 1 & 554 & 372 & 566 & 90.61 & 92.20 & 86.57 & 1.44 & 0.81 & 2.3 \\
\hline 2 & 309 & 438 & 464 & 87.38 & 94.52 & 90.09 & 6.15 & 0.23 & 4.09 \\
\hline 3 & 427 & 555 & 390 & 96.02 & 90.81 & 97.95 & 0 & 0.18 & 0 \\
\hline 4 & 551 & 540 & 507 & 85.48 & 88.33 & 85.01 & 0 & 1.11 & 0 \\
\hline 5 & 536 & 499 & 434 & 89.74 & 86.37 & $\begin{array}{l}97.24 \\
\end{array}$ & 2.24 & 3.41 & 3.69 \\
\hline Mean & & & & $\mathbf{8 9 . 8 5}$ & 90.45 & 91.37 & 3.28 & 1.15 & 3.36 \\
\hline SD & & & & 4.00 & 3.20 & 5.97 & 2.52 & 1.32 & 0.94 \\
\hline RSU* $^{*}$ & & & & 4.97 & 3.97 & 7.41 & & & \\
\hline
\end{tabular}


Table 40 Table presenting the amount of uranium contamination removed from Stainless Steel surfaces

\begin{tabular}{|c|c|c|c|c|c|c|c|c|c|}
\hline & \multicolumn{3}{|c|}{$\begin{array}{c}\text { Radioactivity on Surface and Decontamination } \\
\text { Efficiency }\end{array}$} & \multicolumn{3}{c|}{$\begin{array}{c}\text { Collection on Air Filter } \\
\text { Sample }\end{array}$} \\
\hline \multicolumn{3}{|c|}{$\begin{array}{c}\text { Initial Radioactivity } \\
\text { (Net Counts in 2 } \\
\text { Minutes) }\end{array}$} & \multicolumn{2}{c|}{$\begin{array}{c}\text { Decontamination } \\
\text { Efficiency (\%) }\end{array}$} & $\begin{array}{c}\text { Radioactivity on Filter/Total } \\
\text { Initial Radioactivity on } \\
\text { Surface (\%) }\end{array}$ \\
\hline 1 & 2 & 3 & 4 & 5 & 6 & 7 & 8 & 9 & 10 \\
\hline Exp. & W3 & W1 & W2 & W3 & W1 & W2 & W3 & W1 & W2 \\
\hline 1 & 395 & 492 & 336 & 57.77 & 53.08 & 69.12 & 0.89 & 0.31 & 0 \\
\hline 2 & 314 & 451 & 351 & 48.46 & 71.23 & 85.84 & 0 & 0.11 & 0.29 \\
\hline 3 & 341 & 366 & 368 & 69.28 & 73.29 & 90.30 & 0.15 & 1.09 & 0.27 \\
\hline 4 & 328 & 330 & 379 & 39.37 & 63.40 & 80.28 & 0.92 & 0.15 & 0.26 \\
\hline 5 & 301 & 307 & 329 & 41.57 & 66.85 & 74.85 & 0.50 & 0.16 & 0.30 \\
\hline Mean & & & & $\mathbf{5 1 . 2 9}$ & $\mathbf{6 5 . 5 7}$ & $\mathbf{8 0 . 0 8}$ & $\mathbf{0 . 6 1}$ & $\mathbf{0 . 3 6}$ & $\mathbf{0 . 2 8}$ \\
\hline SD & & & & $\mathbf{4 . 2 3}$ & $\mathbf{2 . 8 5}$ & $\mathbf{5 . 4 0}$ & $\mathbf{0 . 3 6}$ & $\mathbf{0 . 4 1}$ & $\mathbf{0 . 0 2}$ \\
\hline RSU* & & & & $\mathbf{5 . 2 5}$ & $\mathbf{3 . 5 4}$ & $\mathbf{6 . 7 1}$ & & & \\
\hline
\end{tabular}

${ }^{*}$ Random Standard Uncertainty $=\frac{s}{\sqrt{N}} \mathrm{t}_{95,4}$ 
9.3 Summary of the data collected to evaluate the efficiency of the detectors

\begin{tabular}{|c|c|c|c|}
\hline \multirow{2}{*}{\multicolumn{2}{|c|}{ Eberline-SHP probe AB 380}} & \multirow{2}{*}{\multicolumn{2}{|c|}{ Cobra Packard 5003 detector }} \\
\hline & & & \\
\hline Surface & Activity per surface (cpm) & Test No. & $\begin{array}{l}\text { 10ul of solution of particles } \\
\text { of } 200 \text { mesh }\end{array}$ \\
\hline 1 & 23838 & 1 & 45951 \\
\hline 2 & 21200 & 2 & 43730 \\
\hline 3 & 22829 & 3 & 43730 \\
\hline 4 & 21919 & 4 & 41650 \\
\hline 5 & 22916 & 5 & 40149 \\
\hline 6 & 22571 & 6 & 38049 \\
\hline 7 & 25316 & 7 & 39829 \\
\hline 8 & 25193 & 8 & 34188 \\
\hline 9 & 23890 & 9 & 39029 \\
\hline 10 & 24828 & 10 & 37669 \\
\hline 11 & 20567 & 11 & 35808 \\
\hline 12 & 24118 & 12 & 41009 \\
\hline 13 & 24414 & 13 & 38849 \\
\hline 14 & 20646 & 14 & 43190 \\
\hline 15 & 23338 & 15 & 48811 \\
\hline 16 & 23967 & 16 & 48611 \\
\hline 17 & 26477 & Average & 41266 \\
\hline 18 & 20183 & Stdev & 4213 \\
\hline 19 & 23300 & CV (\%) & 10 \\
\hline 20 & 19935 & \multirow{3}{*}{\multicolumn{2}{|c|}{ Time of measurement 1:23 pm }} \\
\hline 21 & 22450 & & \\
\hline 22 & 25480 & & \\
\hline Average & 23153 & \multirow{2}{*}{\multicolumn{2}{|c|}{ Time elapsed $26.51 \mathrm{hrs}$}} \\
\hline Stdev & 1837 & & \\
\hline CV(\%) & 8 & & \\
\hline \multicolumn{2}{|c|}{ Time of measurement 10:52 am } & & \\
\hline \multicolumn{2}{|c|}{ Time elapsed $24.08 \mathrm{hrs}$} & & \\
\hline
\end{tabular}


9.4 Time correction of the values collected to evaluated the contamination removed by gamma detection

\begin{tabular}{|c|c|c|c|c|c|c|}
\hline \multicolumn{7}{|c|}{ Time Correction } \\
\hline Order & Time & $\begin{array}{c}\text { Time } \\
\text { difference } \\
\text { (min) }\end{array}$ & Time (h) & $\begin{array}{c}\text { Initial } \\
\text { Counts } \\
\text { (*kcpm) }\end{array}$ & $\begin{array}{l}\text { Corrected } \\
\text { Counts } \\
\text { (*kcpm) }\end{array}$ & $\begin{array}{c}\text { Efficiency of } \\
\text { Contamination } \\
\text { Removal (\%) }\end{array}$ \\
\hline \multirow[t]{2}{*}{1} & $10: 04$ & $0: 00$ & 0.00 & 15.28 & 15.28 & \multirow{2}{*}{68.7} \\
\hline & $10: 06$ & $0: 02$ & 0.03 & 4.77 & 4.79 & \\
\hline \multirow[t]{2}{*}{2} & $10: 07$ & $0: 03$ & 0.05 & 17.07 & 17.17 & \multirow[t]{2}{*}{68.1} \\
\hline & 10:09 & $0: 05$ & 0.08 & 5.41 & 5.47 & \\
\hline \multirow[t]{2}{*}{3} & $10: 10$ & $0: 06$ & 0.10 & 8.41 & 8.51 & \multirow[t]{2}{*}{5.8} \\
\hline & $10: 12$ & $0: 08$ & 0.13 & 7.88 & 8.02 & \\
\hline \multirow[t]{2}{*}{4} & $10: 14$ & $0: 10$ & 0.16 & 11.89 & 12.13 & \multirow[t]{2}{*}{36.7} \\
\hline & $10: 16$ & $0: 12$ & 0.20 & 7.51 & 7.68 & \\
\hline \multirow[t]{2}{*}{5} & $10: 18$ & $0: 14$ & 0.23 & 8.20 & 8.43 & \multirow[t]{2}{*}{8.4} \\
\hline & $10: 20$ & $0: 16$ & 0.26 & 7.49 & 7.72 & \\
\hline \multirow[t]{2}{*}{6} & $10: 22$ & $0: 18$ & 0.30 & 14.42 & 14.92 & \multirow[t]{2}{*}{66.8} \\
\hline & $10: 24$ & $0: 20$ & 0.33 & 4.77 & 4.95 & \\
\hline \multirow[t]{2}{*}{7} & $10: 26$ & $0: 22$ & 0.36 & 17.34 & 18.09 & \multirow[t]{2}{*}{69.8} \\
\hline & $10: 28$ & $0: 24$ & 0.40 & 5.23 & 5.47 & \\
\hline \multirow[t]{2}{*}{8} & $10: 30$ & $0: 26$ & 0.43 & 8.78 & 9.23 & \multirow[t]{2}{*}{6.7} \\
\hline & $10: 32$ & $0: 28$ & 0.47 & 8.16 & 8.61 & \\
\hline \multirow[t]{2}{*}{9} & $10: 33$ & $0: 29$ & 0.48 & 15.55 & 16.44 & \multirow[t]{2}{*}{75.8} \\
\hline & $10: 35$ & $0: 31$ & 0.51 & 3.75 & 3.98 & \\
\hline \multirow[t]{2}{*}{10} & $10: 37$ & $0: 33$ & 0.55 & 12.92 & 13.76 & \multirow[t]{2}{*}{36.0} \\
\hline & $10: 39$ & $0: 35$ & 0.58 & 8.23 & 8.8 & \\
\hline \multirow[t]{2}{*}{11} & $10: 41$ & $0: 37$ & 0.61 & 9.45 & 10.14 & \multirow[t]{2}{*}{40.6} \\
\hline & $10: 43$ & $0: 39$ & 0.65 & 5.59 & 6.02 & \\
\hline \multirow[t]{2}{*}{12} & $10: 44$ & $0: 40$ & 0.66 & 16.01 & 17.29 & \multirow[t]{2}{*}{75.4} \\
\hline & $10: 46$ & $0: 42$ & 0.70 & 3.92 & 4.25 & \\
\hline \multirow[t]{2}{*}{13} & $10: 48$ & $0: 44$ & 0.73 & 8.90 & 9.68 & \multirow[t]{2}{*}{42.0} \\
\hline & $10: 50$ & $0: 46$ & 0.76 & 5.14 & 5.61 & \\
\hline \multirow[t]{2}{*}{14} & $10: 52$ & $0: 48$ & 0.80 & 11.46 & 12.56 & 72.0 \\
\hline & $10: 54$ & $0: 50$ & 0.83 & 3.19 & 3.52 & \\
\hline 15 & $10: 55$ & $0: 51$ & 0.85 & 8.58 & 9.46 & 9.9 \\
\hline & $10: 57$ & $0: 53$ & 0.88 & 7.70 & 8.52 & \\
\hline 16 & $10: 58$ & $0: 54$ & 0.90 & 15.50 & 17.19 & 80.7 \\
\hline & $11: 00$ & $0: 56$ & 0.93 & 2.97 & 3.32 & \\
\hline 17 & $11: 02$ & $0: 58$ & 0.97 & 8.57 & 9.58 & 4.9 \\
\hline & 11:04 & $1: 00$ & 1.0 & 8.12 & 9.11 & \\
\hline
\end{tabular}




\begin{tabular}{|c|c|c|c|c|c|c|}
\hline Order & Time & $\begin{array}{c}\text { Time } \\
\text { difference } \\
(\text { min) }\end{array}$ & Time (h) & $\begin{array}{c}\text { Initial } \\
\text { Counts } \\
\text { (*kcpm) }\end{array}$ & $\begin{array}{c}\text { Corrected } \\
\text { Counts } \\
\text { (*kcpm) }\end{array}$ & $\begin{array}{c}\text { Efficiency of } \\
\text { Contamination } \\
\text { Removal (\%) }\end{array}$ \\
\hline \multirow[t]{2}{*}{18} & $11: 06$ & $1: 02$ & 1.03 & 10.62 & 11.96 & \multirow[t]{2}{*}{87.7} \\
\hline & $11: 07$ & $1: 03$ & 1.05 & 1.30 & 1.47 & \\
\hline \multirow[t]{2}{*}{19} & $11: 09$ & $1: 05$ & 1.08 & 10.03 & 11.36 & \multirow[t]{2}{*}{47.6} \\
\hline & 11:11 & 1:07 & 1.12 & 5.23 & 5.95 & \\
\hline \multirow[t]{2}{*}{20} & $11: 13$ & $1: 09$ & 1.15 & 13.58 & $\mathbf{1 5 . 5}$ & \multirow[t]{2}{*}{77.4} \\
\hline & $11: 15$ & $1: 11$ & 1.18 & 3.06 & 3.5 & \\
\hline \multirow[t]{2}{*}{21} & $11: 16$ & $1: 12$ & 1.20 & 13.30 & 15.27 & \multirow[t]{2}{*}{73.1} \\
\hline & $11: 18$ & $1: 14$ & 1.23 & 3.57 & 4.11 & \\
\hline \multirow[t]{2}{*}{22} & $11: 20$ & $1: 16$ & 1.27 & 11.49 & 13.29 & \multirow[t]{2}{*}{52.1} \\
\hline & $11: 21$ & $1: 17$ & 1.28 & 5.49 & 6.36 & \\
\hline \multirow[t]{2}{*}{23} & $11: 23$ & $1: 19$ & 1.32 & 10.62 & 12.36 & \multirow[t]{2}{*}{74.0} \\
\hline & $11: 25$ & $1: 21$ & 1.35 & 2.75 & 3.21 & \\
\hline \multirow[t]{2}{*}{24} & $11: 26$ & $1: 22$ & 1.37 & 6.59 & 7.71 & \multirow[t]{2}{*}{8.3} \\
\hline & $11: 28$ & $1: 24$ & 1.40 & 6.02 & 7.07 & \\
\hline
\end{tabular}

* kcpm: kilo counts per minute

9.4.1 Model adequacy checking direct method

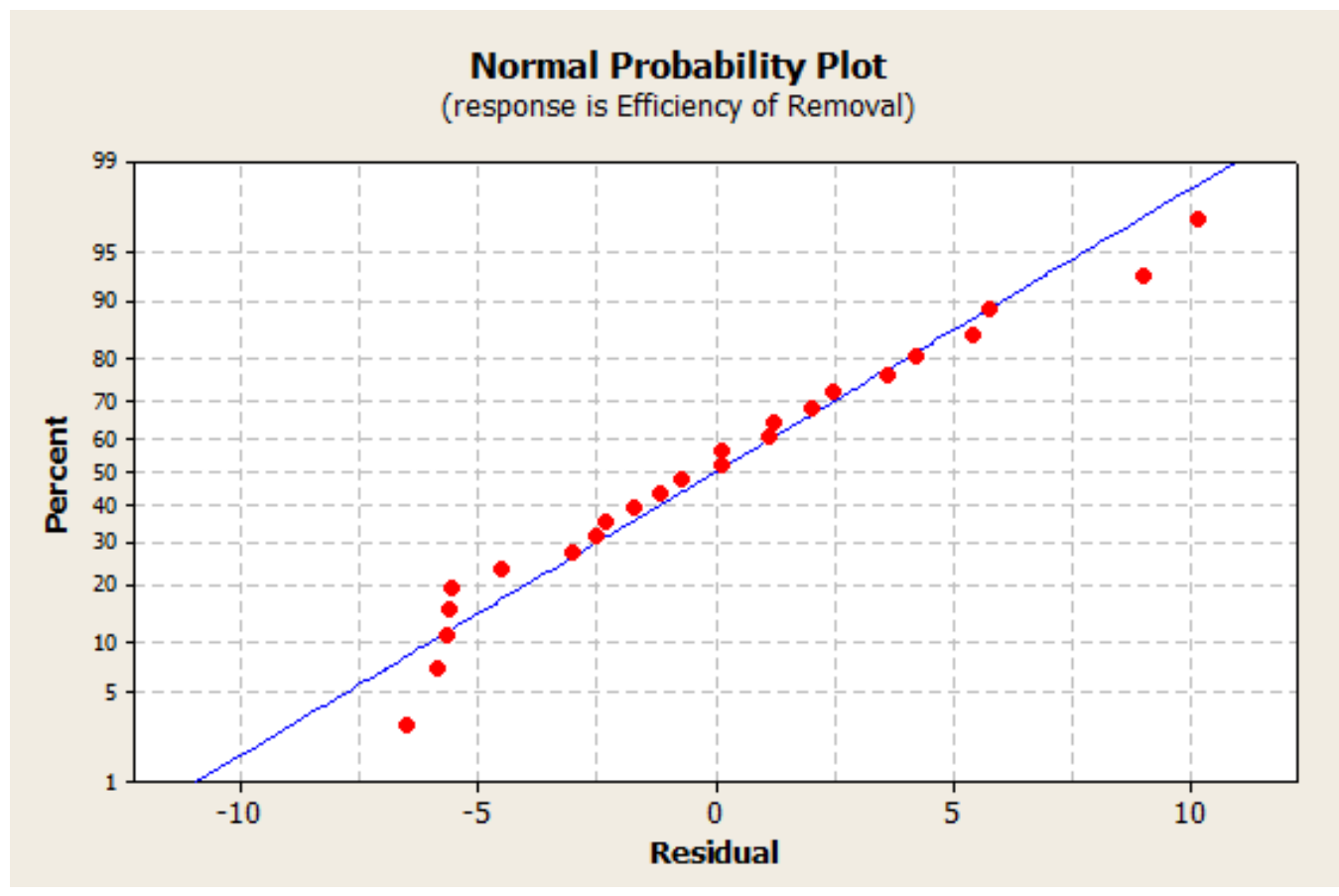

Figure 52 Normal probability plot of the Residuals 


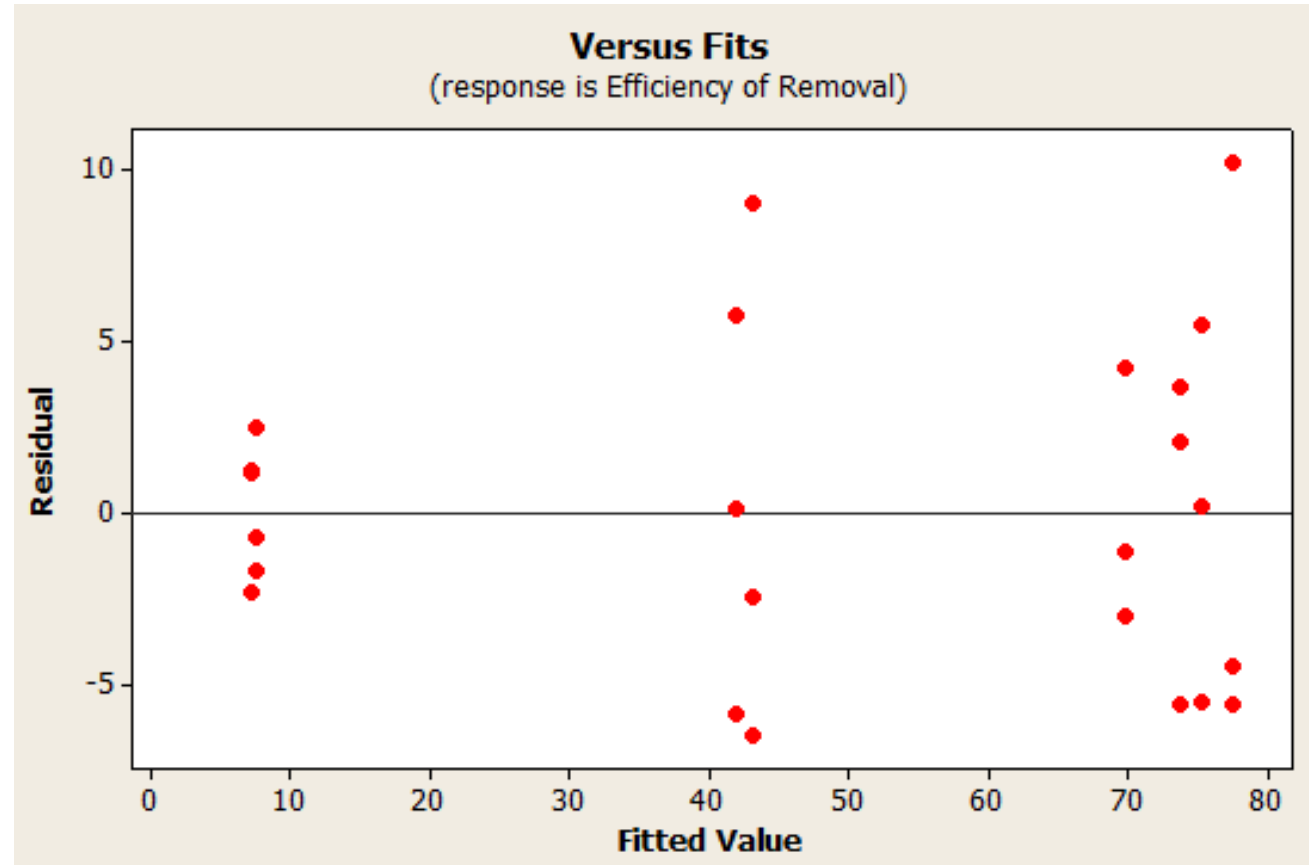

Figure 53 Residual versus fitted value

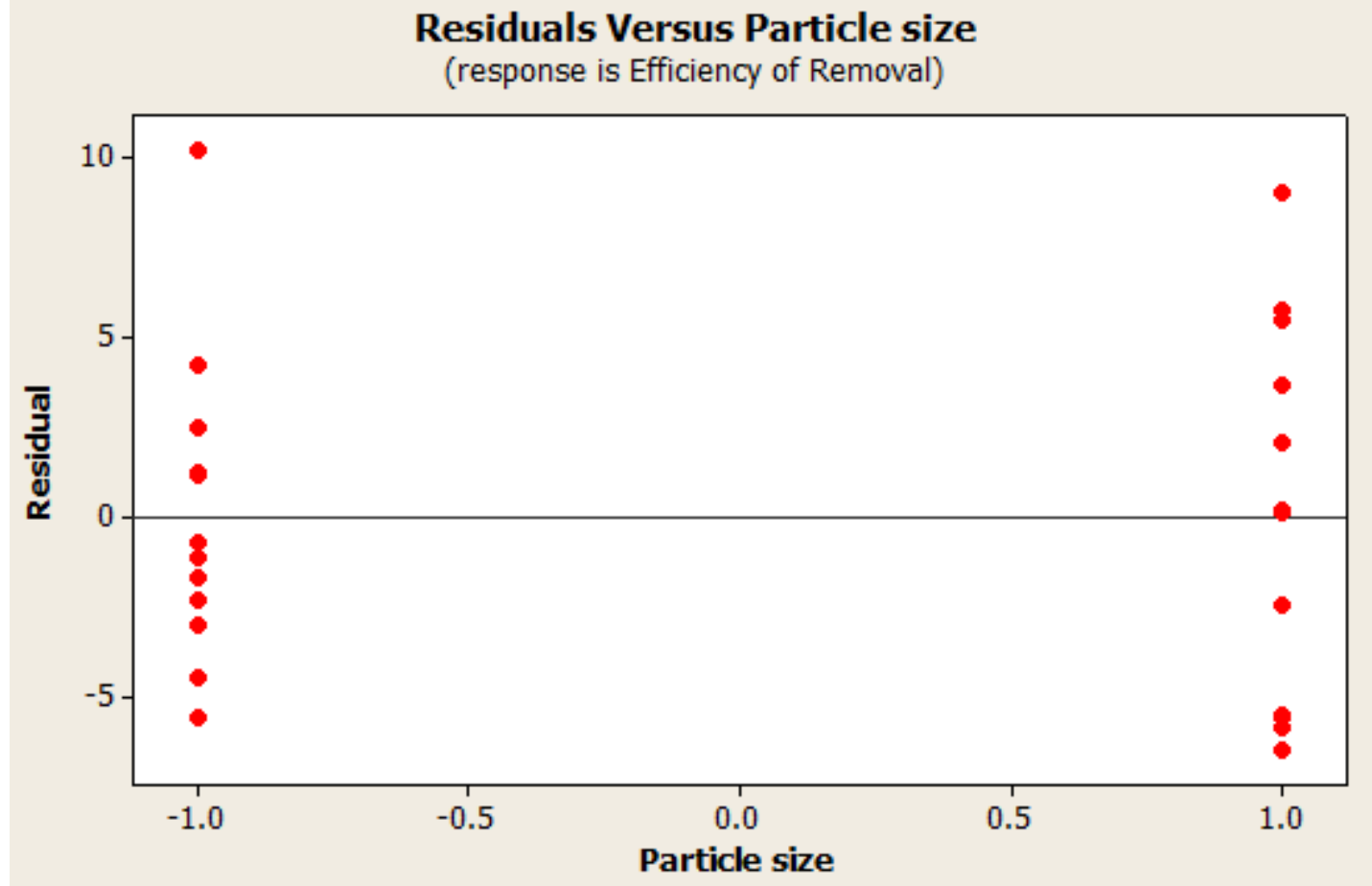

Figure 54 Residual versus particle size of the contaminant 


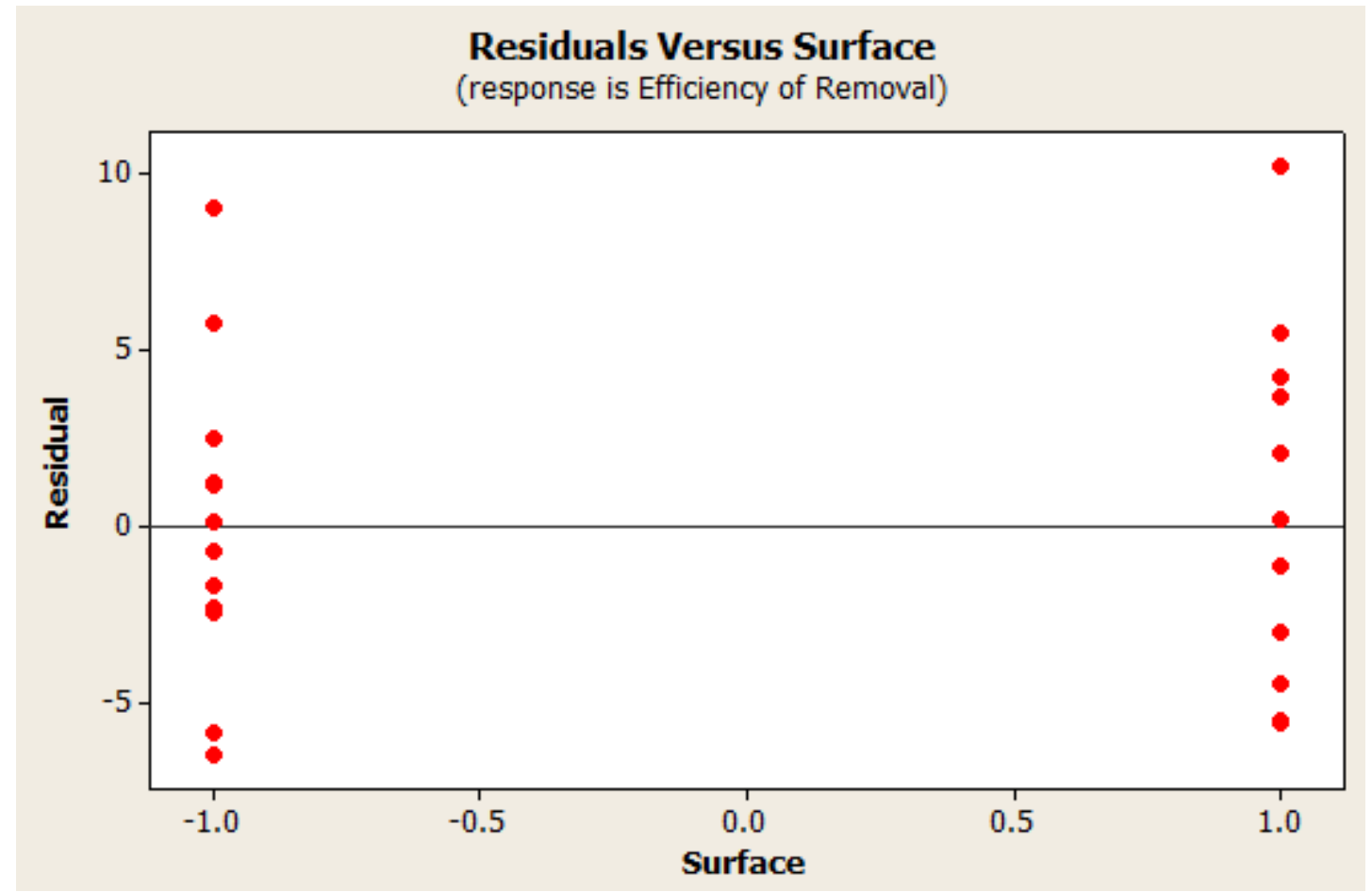

Figure 55 Residual versus surface roughness 
9.4.2 Model adequacy checking indirect method

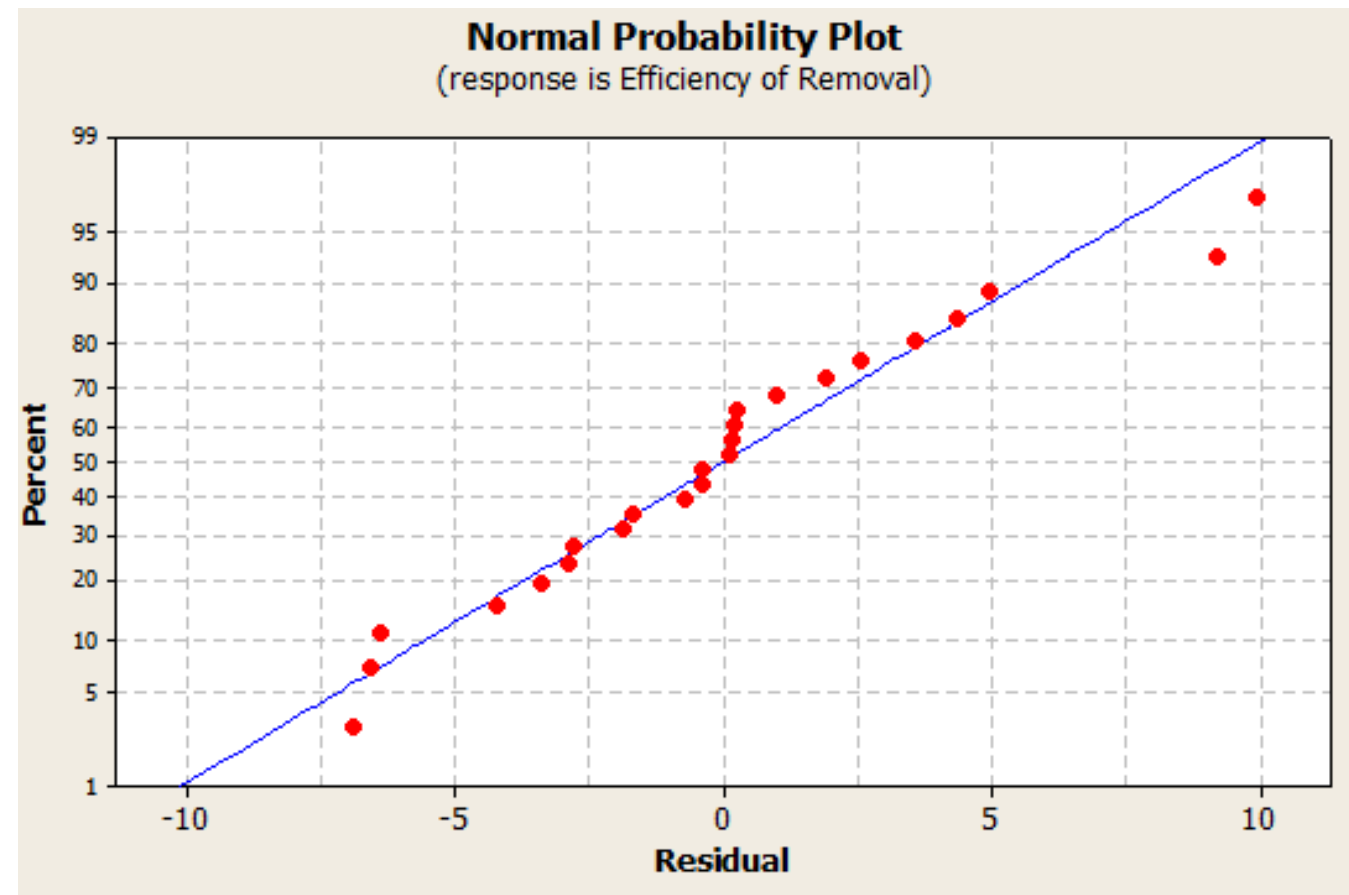

Figure 56 Normal probability plot of the residual

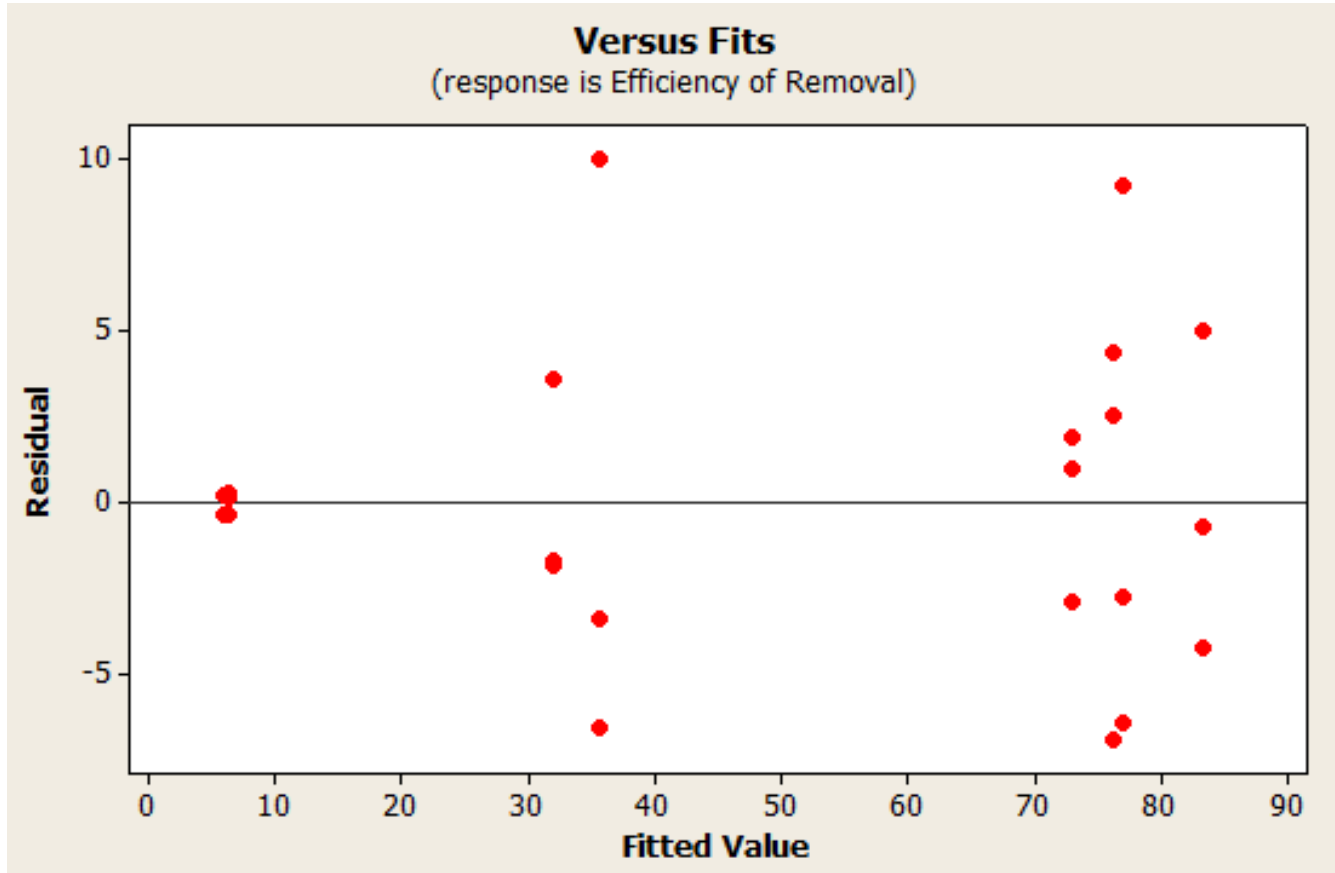

Figure 57 Residual versus fitted value 


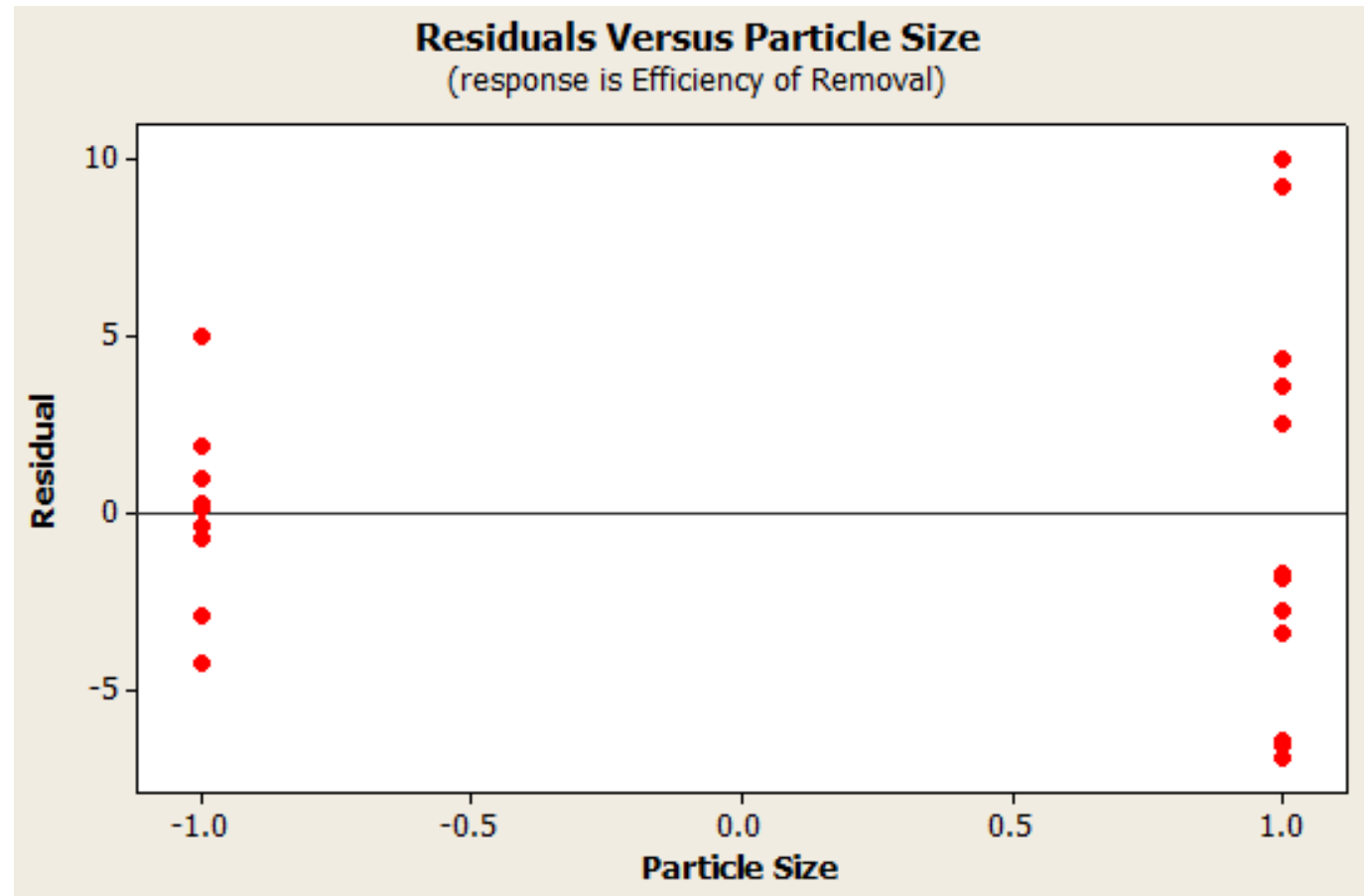

Figure 58 Residual versus particle size of the contaminant

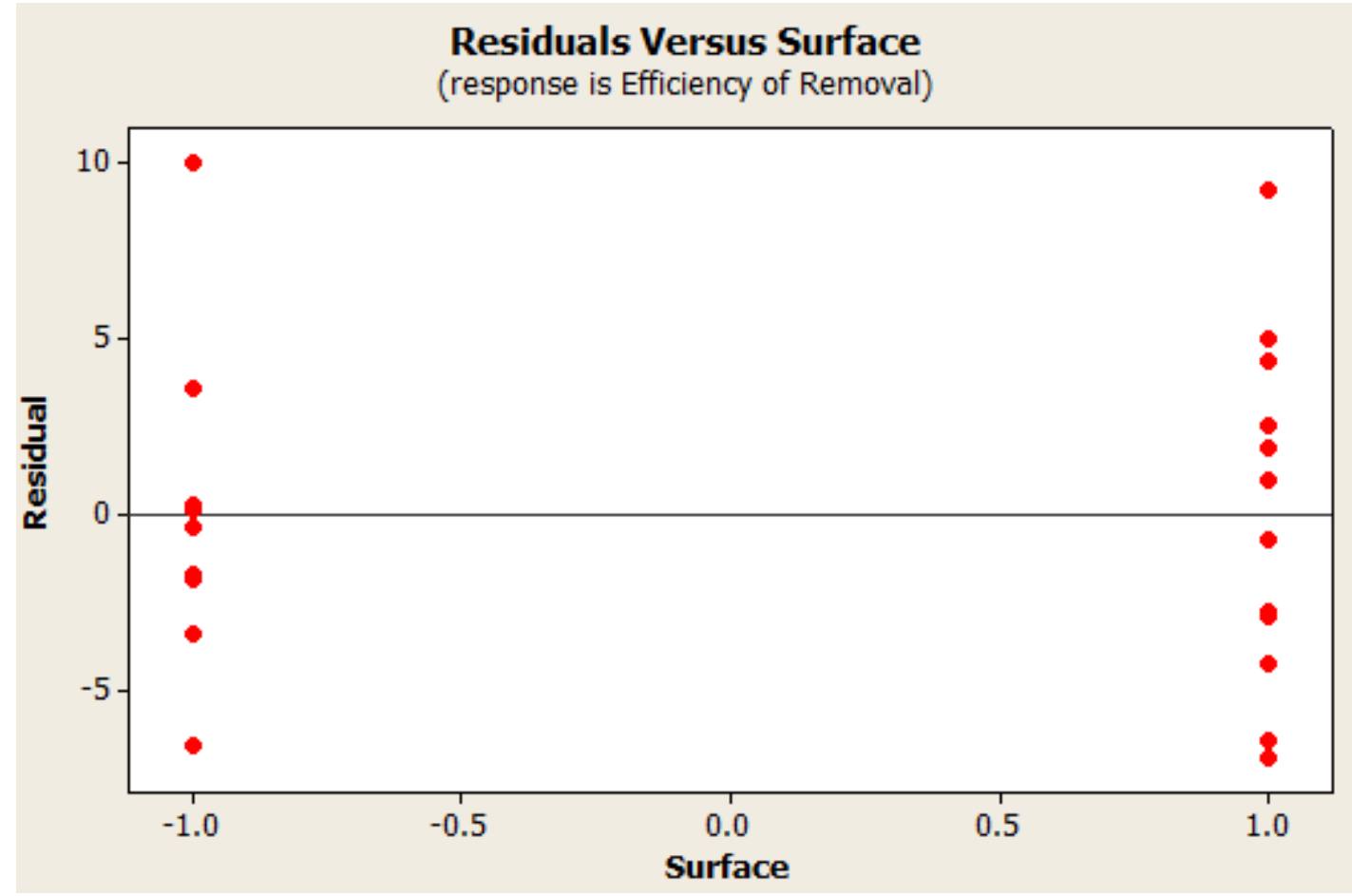

Figure 59 Residual versus surface roughness 
9.5 Time correction for data collected study the surface energy by gamma radiation detection

\begin{tabular}{|c|c|c|c|c|c|c|}
\hline \multicolumn{7}{|c|}{ Time Correction } \\
\hline Order & Time & \begin{tabular}{|l|} 
Time \\
difference \\
(min)
\end{tabular} & Time (h) & $\begin{array}{l}\text { Initial } \\
\text { Counts } \\
\text { (*kcpm) }\end{array}$ & $\begin{array}{l}\text { Corrected } \\
\text { Counts } \\
\left({ }^{* k c p m}\right)\end{array}$ & $\begin{array}{c}\text { Efficiency of } \\
\text { Contamination } \\
\text { Removal (\%) }\end{array}$ \\
\hline \multirow[t]{2}{*}{1} & $10: 52$ & $0: 00$ & 0.00 & 23.840 & 23.840 & \multirow[t]{2}{*}{68.6} \\
\hline & $10: 54$ & $0: 02$ & 0.03 & 7.458 & 7.487 & \\
\hline \multirow[t]{2}{*}{2} & $10: 56$ & $0: 04$ & 0.07 & 21.038 & 21.2 & \multirow[t]{2}{*}{82.6} \\
\hline & $10: 59$ & $0: 07$ & 0.12 & 3.638 & 3.687 & \\
\hline \multirow[t]{2}{*}{3} & 11:01 & 0:09 & 0.15 & 22.438 & 22.829 & \multirow[t]{2}{*}{86} \\
\hline & $11: 03$ & $0: 11$ & 0.18 & 3.128 & 3.195 & \\
\hline \multirow[t]{2}{*}{4} & $11: 06$ & $0: 14$ & 0.23 & 21.338 & 21.919 & \multirow[t]{2}{*}{68.7} \\
\hline & $11: 08$ & $0: 16$ & 0.27 & 6.658 & 6.866 & \\
\hline \multirow[t]{2}{*}{5} & $11: 10$ & $0: 18$ & 0.30 & 22.138 & 22.916 & \multirow[t]{2}{*}{68.0} \\
\hline & $11: 12$ & $0: 20$ & 0.33 & 7.048 & 7.324 & \\
\hline \multirow[t]{2}{*}{6} & $11: 14$ & $0: 22$ & 0.37 & 21.638 & 22.571 & \multirow[t]{2}{*}{92.1} \\
\hline & $11: 16$ & $0: 24$ & 0.40 & 1.698 & 1.778 & \\
\hline \multirow[t]{2}{*}{7} & $11: 19$ & $0: 27$ & 0.45 & 24.038 & 25.316 & \multirow[t]{2}{*}{77.0} \\
\hline & $11: 21$ & $0: 29$ & 0.48 & 5.498 & 5.813 & \\
\hline \multirow[t]{2}{*}{8} & $11: 23$ & $0: 31$ & 0.52 & 23.738 & 25.193 & \multirow[t]{2}{*}{77.8} \\
\hline & $11: 25$ & $0: 33$ & 0.55 & 5.248 & 5.591 & \\
\hline \multirow[t]{2}{*}{9} & $11: 27$ & $0: 35$ & 0.58 & 22.338 & 23.89 & \multirow[t]{2}{*}{87.2} \\
\hline & $11: 29$ & $0: 37$ & 0.61 & 2.848 & 3.058 & \\
\hline \multirow[t]{2}{*}{10} & $11: 31$ & $0: 39$ & 0.65 & 23.038 & 24.828 & \multirow[t]{2}{*}{76.6} \\
\hline & $11: 33$ & $0: 41$ & 0.68 & 5.368 & 5.807 & \\
\hline \multirow[t]{2}{*}{11} & $11: 35$ & $0: 43$ & 0.72 & 18.938 & 20.567 & \multirow[t]{2}{*}{69.3} \\
\hline & $11: 37$ & $0: 45$ & 0.75 & $\begin{array}{l}5.788 \\
\end{array}$ & 6.31 & \\
\hline \multirow[t]{2}{*}{12} & $11: 39$ & $0: 47$ & 0.78 & 22.038 & 24.118 & \multirow[t]{2}{*}{82.2} \\
\hline & $11: 41$ & $0: 49$ & 0.82 & 3.918 & 4.304 & \\
\hline \multirow[t]{2}{*}{13} & $11: 43$ & $0: 51$ & 0.85 & 22.138 & 24.414 & \multirow[t]{2}{*}{77.8} \\
\hline & $11: 45$ & $0: 53$ & 0.88 & 4.888 & 5.411 & \\
\hline \multirow[t]{2}{*}{14} & $11: 47$ & $0: 55$ & 0.92 & 18.578 & 20.646 & 73.9 \\
\hline & $11: 49$ & $0: 57$ & 0.95 & 4.828 & 5.386 & \\
\hline 15 & $11: 51$ & $0: 59$ & 0.98 & 23.338 & 26.136 & 78.3 \\
\hline & $11: 53$ & 1:01 & 1.02 & $\begin{array}{l}5.038 \\
\end{array}$ & 5.664 & \\
\hline 16 & $11: 55$ & $1: 03$ & 1.05 & 21.238 & 23.967 & 79.4 \\
\hline & $11: 58$ & $1: 06$ & 1.10 & 4.358 & 4.946 & \\
\hline 17 & $12: 00$ & $1: 08$ & 1.13 & 23.238 & 26.477 & 66.9 \\
\hline & $12: 02$ & $1: 10$ & 1.17 & 7.658 & 8.759 & \\
\hline
\end{tabular}




\begin{tabular}{|c|c|c|c|c|c|c|}
\hline Order & Time & $\begin{array}{l}\text { Time } \\
\text { difference } \\
\text { (min) }\end{array}$ & Time (h) & $\begin{array}{l}\text { Initial } \\
\text { Counts } \\
\text { (*kcpm) }\end{array}$ & $\begin{array}{l}\text { Corrected } \\
\text { Counts } \\
\text { (*kcpm) }\end{array}$ & $\begin{array}{c}\text { Efficiency of } \\
\text { Contamination } \\
\text { Removal (\%) }\end{array}$ \\
\hline \multirow[t]{2}{*}{18} & $12: 04$ & $1: 12$ & 1.2 & 17.578 & 20.183 & \multirow[t]{2}{*}{68.7} \\
\hline & $12: 06$ & $1: 14$ & 1.23 & 5.478 & 6.314 & \\
\hline \multirow[t]{2}{*}{19} & $12: 08$ & $1: 16$ & 1.27 & 20.138 & 23.300 & \multirow[t]{2}{*}{58.1} \\
\hline & $12: 10$ & $1: 18$ & 1.30 & 8.398 & 9.754 & \\
\hline \multirow[t]{2}{*}{20} & $12: 12$ & $1: 20$ & 1.33 & 17.098 & 19.935 & \multirow[t]{2}{*}{70.0} \\
\hline & $12: 14$ & $1: 22$ & 1.37 & 5.108 & 5.978 & \\
\hline \multirow[t]{2}{*}{21} & $12: 19$ & $1: 27$ & 1.45 & 18.998 & 22.450 & \multirow[t]{2}{*}{88.8} \\
\hline & $12: 21$ & $1: 29$ & 1.48 & 2.128 & 2.524 & \\
\hline \multirow[t]{2}{*}{22} & $12: 22$ & $1: 30$ & 1.50 & 21.438 & 25.48 & \multirow[t]{2}{*}{61.5} \\
\hline & $12: 24$ & $1: 32$ & 1.53 & 8.228 & 9.817 & \\
\hline \multirow[t]{2}{*}{23} & $12: 26$ & $1: 34$ & 1.57 & 13.698 & 16.406 & \multirow[t]{2}{*}{91.1} \\
\hline & $12: 28$ & $1: 36$ & 1.60 & 1.208 & 1.452 & \\
\hline \multirow[t]{2}{*}{24} & $12: 30$ & $1: 38$ & 1.63 & 15.738 & 18.994 & \multirow[t]{2}{*}{69.1} \\
\hline & $12: 32$ & $1: 40$ & 1.67 & 4.838 & 5.861 & \\
\hline
\end{tabular}

*kcpm: kilo counts per minute 
9.5.1 Model adequacy checking

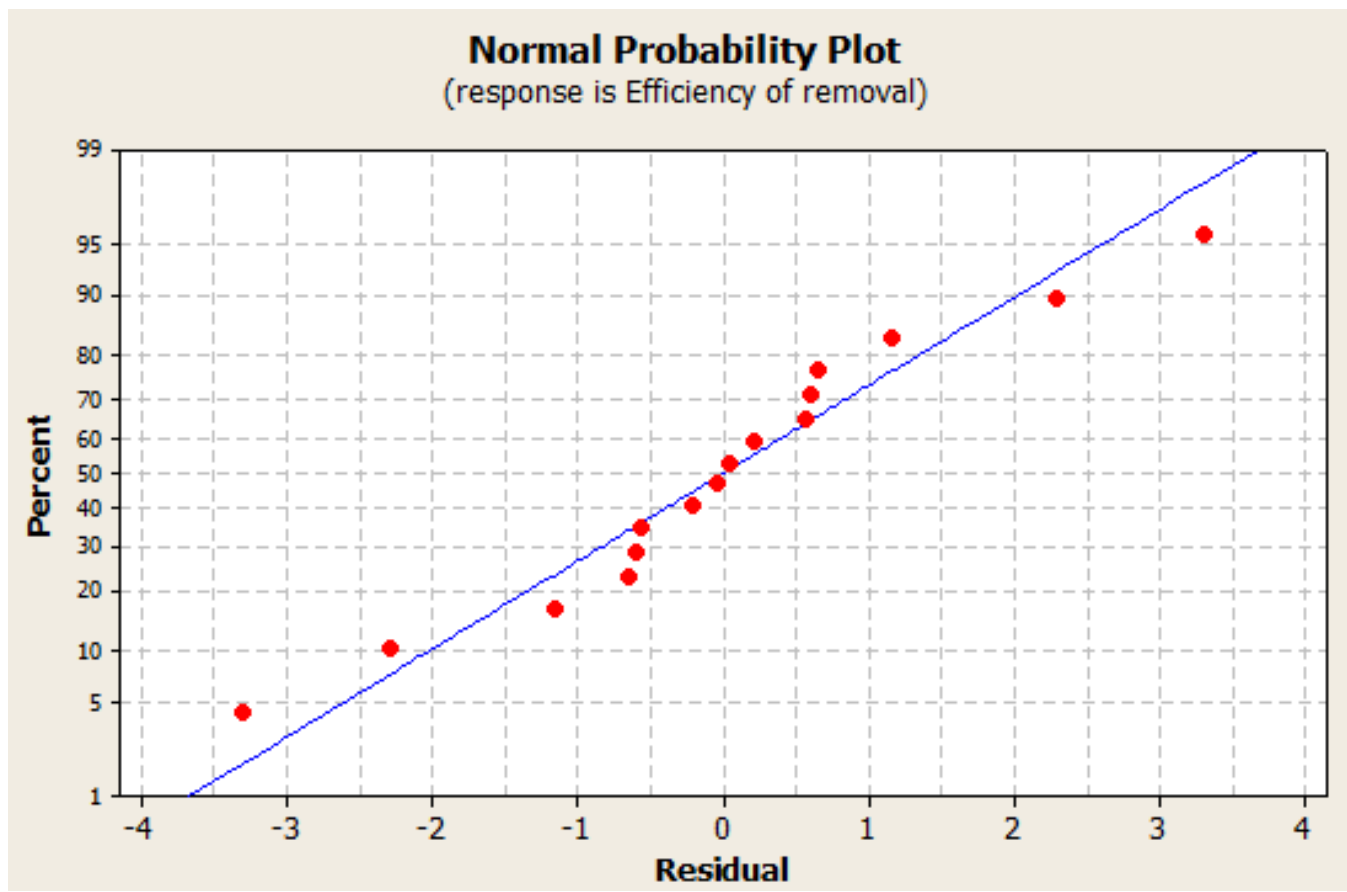

Figure 60 Normal probability plot of the residuals

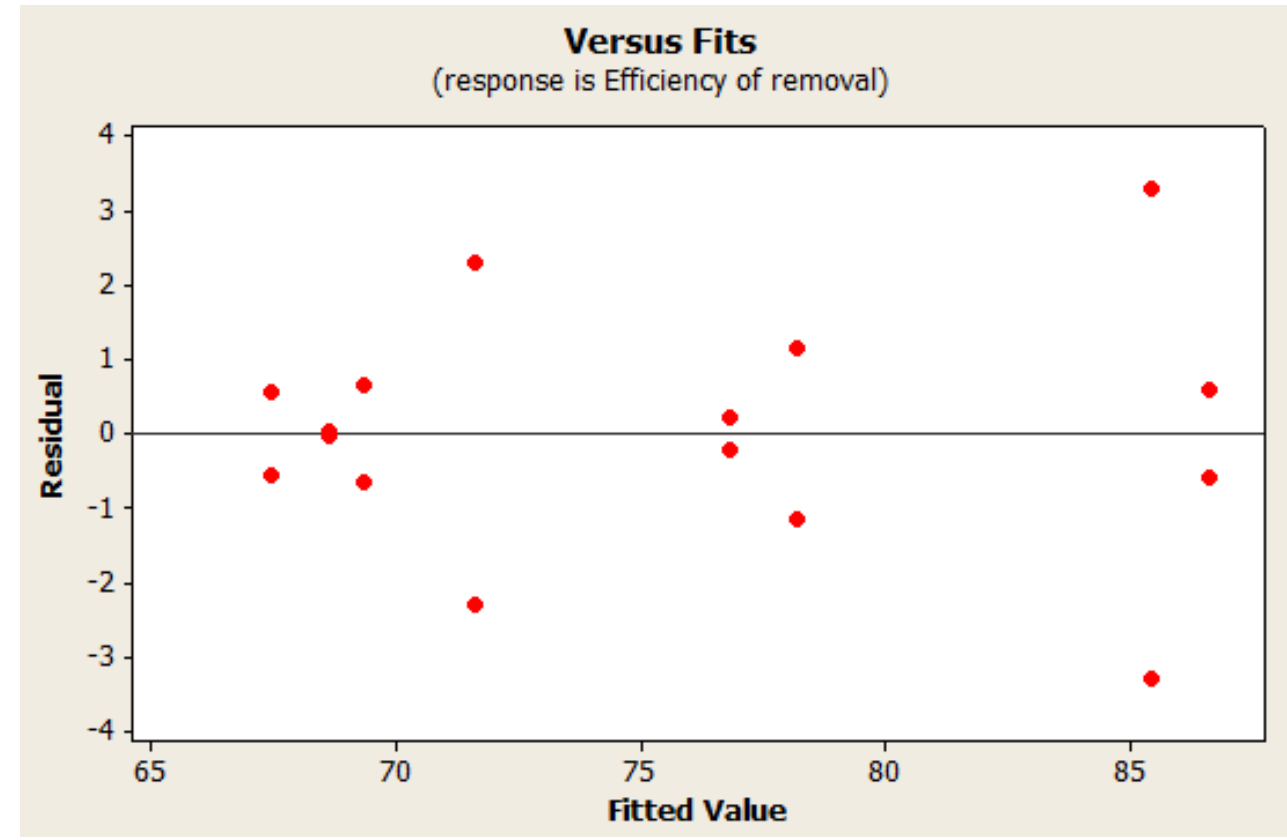

Figure 61 Residual plot versus fitted value 


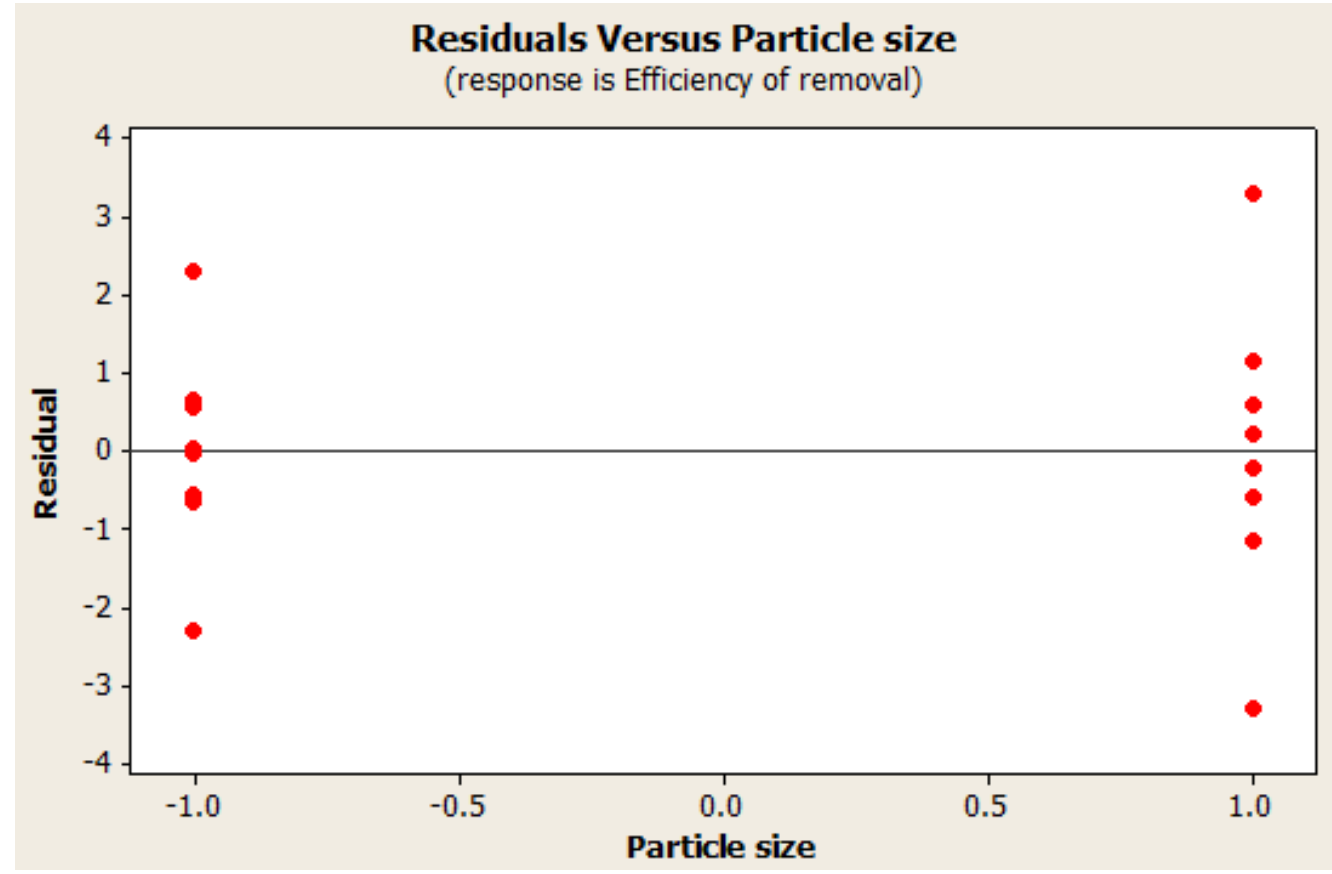

Figure 62 Residual versus particle size of the contaminant

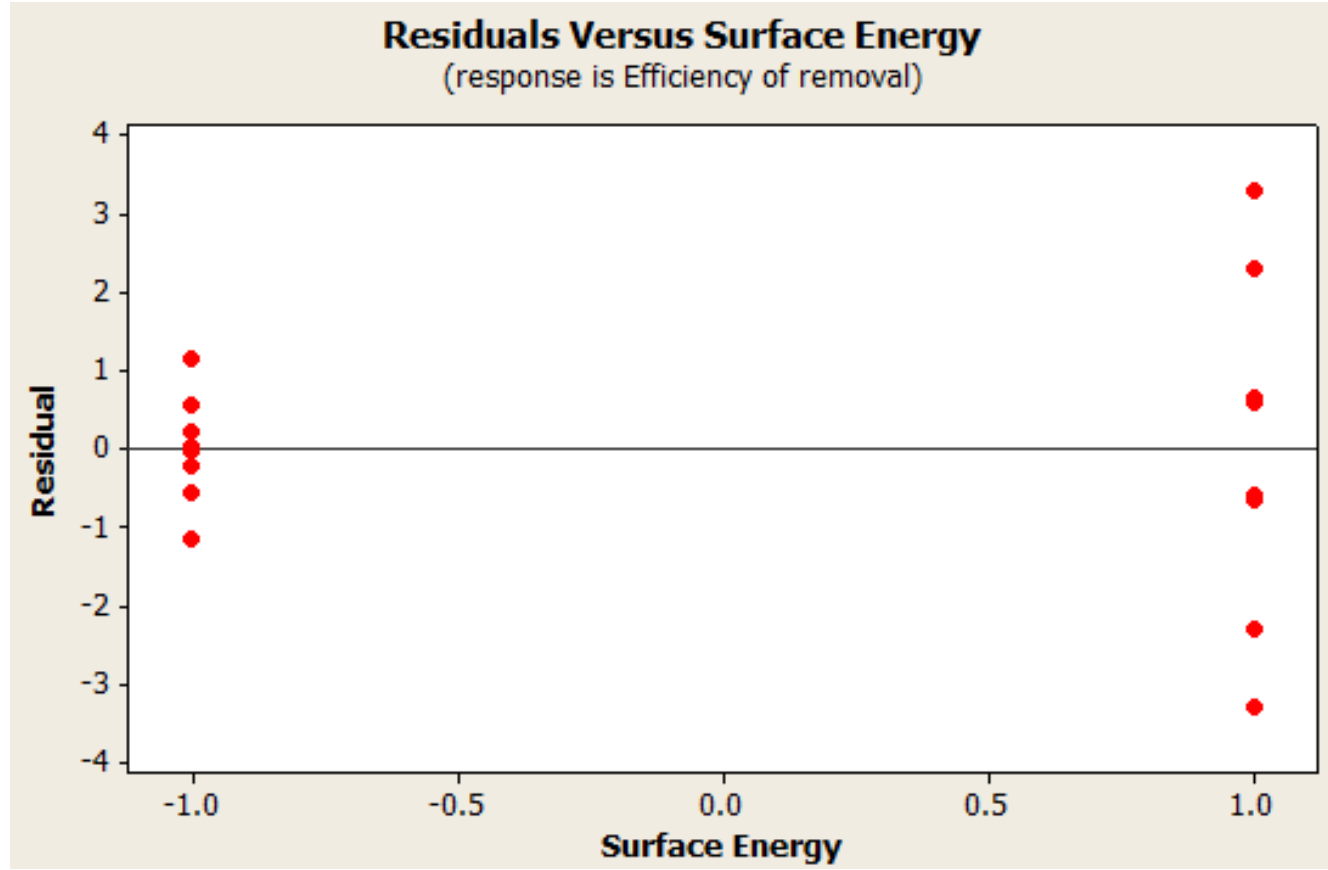

Figure 63 Residual versus surface energy of the surface 